(C) 2006 IEEE. Personal use of this material is permitted. Permission from IEEE must be obtained for all other uses, in any current or future media, including reprinting/republishing this material for advertising or promotional purposes, creating new collective works, for resale or redistribution to servers or lists, or reuse of any copyrighted component of this work in other works. 


\title{
Partial Recovery of Quantum Entanglement
}

\author{
Runyao Duan, Yuan Feng, and Mingsheng Ying
}

\begin{abstract}
Suppose Alice and Bob try to transform an entangled state shared between them into another one by local operations and classical communications. Then in general a certain amount of entanglement contained in the initial state will decrease in the process of transformation. However, an interesting phenomenon called partial entanglement recovery shows that it is possible to recover some amount of entanglement by adding another entangled state and transforming the two entangled states collectively.

In this paper we are mainly concerned with the feasibility of partial entanglement recovery. The basic problem we address is whether a given state is useful in recovering entanglement lost in a specified transformation. In the case where the source and target states of the original transformation satisfy the strict majorization relation, a necessary and sufficient condition for partial entanglement recovery is obtained. For the general case we give two sufficient conditions. We also give an efficient algorithm for the feasibility of partial entanglement recovery in polynomial time.

As applications, we establish some interesting connections between partial entanglement recovery and the generation of maximally entangled states, quantum catalysis, mutual catalysis, and multiple-copy entanglement transformation.
\end{abstract}

Index Terms - Quantum entanglement, Entanglement transformation, Partial entanglement recovery, Entanglement catalysis, Majorization.

\section{INTRODUCTION}

Q UANTUM entanglement is a valuable resource in quantum information processing. It can implement some information processing tasks that cannot be accomplished classically. As a consequence, entanglement has been widely used in quantum cryptography [1], quantum superdense coding [2], and quantum teleportation [3]; see [4], Chapter 12 for an excellent exposition. Due to the great importance of quantum entanglement, a fruitful branch of quantum information theory named quantum entanglement theory is currently being developed.

Since quantum entanglement exists between different subsystems of a composite system shared by spatially separated parties, a natural constraint on the manipulation of entanglement is that the separated parties are only allowed to perform local quantum operations on their own subsystems and to communicate to each other classically (LOCC). Using this restricted set of transformations, the parties are often required to optimally manipulate the entangled state. One of the central

This work was partly supported by the Natural Science Foundation of China (Grant Nos. 60503001, 60321002, and 60305005), and by Tsinghua Basic Research Foundation (Grant No. 052220204). R. Duan acknowledges the financial support of Tsinghua University (Grant No. 052420003).

The authors are with the State Key Laboratory of Intelligent Technology and Systems, Department of Computer Science and Technology, Tsinghua University, Beijing, China 100084. E-mails: dry02@mails.tsinghua.edu.cn (Runyao Duan), feng-y@tsinghua.edu.cn (Yuan Feng), and yingmsh@tsinghua.edu.cn (Mingsheng Ying) problems of quantum entanglement theory is thus to find the conditions for when an entangled state can be transformed into another one using LOCC. This problem can be approached in two different, but complementary, contexts: the finite regime and the asymptotic regime. In the asymptotic regime Bennett and his collaborators [5] proposed a reversible protocol which shows that any two bipartite entangled pure states with infinite copies can be converted into each other without any loss of entropy of entanglement. Since in practice one can only have finitely many copies of an entangled state, it is of great interest to consider the problem of entanglement transformation in a finite (non-asymptotic) setting. Arguably, the most important step in the finite regime was made by Nielsen in [6], where he reported a necessary and sufficient condition for a bipartite entangled pure state to be transformed into another pure one deterministically using LOCC. Suppose two distantly located parties, Alice and Bob, share an entangled state $|\psi\rangle$ in $\mathcal{C}^{n} \otimes \mathcal{C}^{n}$ with Schmidt decomposition $|\psi\rangle=\sum_{i=1}^{n} \sqrt{\alpha_{i}}\left|i_{A}\right\rangle\left|i_{B}\right\rangle$, where $\alpha_{1} \geq \alpha_{2} \geq \cdots \geq \alpha_{n} \geq 0$ are Schmidt coefficients and $\sum_{i=1}^{n} \alpha_{i}=1 .\left|i_{A}\right\rangle$ and $\left|i_{B}\right\rangle$ are orthonormal bases of Alice's and Bob's systems, respectively. Suppose the two parties want to transform $|\psi\rangle$ into another state $|\varphi\rangle$ with Schmidt decomposition $|\varphi\rangle=\sum_{i=1}^{n} \sqrt{\beta_{i}}\left|i_{A}^{\prime}\right\rangle\left|i_{B}^{\prime}\right\rangle$, where $\beta_{1} \geq \beta_{2} \geq$ $\cdots \geq \beta_{n} \geq 0$ and $\sum_{i=1}^{n} \beta_{i}=1$. The orthonormal bases $\left|i_{A}\right\rangle$ and $\left|i_{A}^{\prime}\right\rangle$ (similarly, $\left|i_{B}\right\rangle$ and $\left|i_{B}^{\prime}\right\rangle$ ) are not necessarily the same. For the sake of convenience, we write $\psi=\left(\alpha_{1}, \cdots, \alpha_{n}\right)$ and $\varphi=\left(\beta_{1}, \cdots, \beta_{n}\right)$ for the respective ordered Schmidt coefficient vectors of $|\psi\rangle$ and $|\varphi\rangle$. Nielsen proved that Alice and Bob can achieve this transformation of $|\psi\rangle$ to $|\varphi\rangle$ with certainty using LOCC, written $|\psi\rangle \rightarrow|\varphi\rangle$, if and only if $\psi \prec \varphi$. Here the symbol ' $\prec$ ' stands for 'majorization relation', and $\psi \prec \varphi$ holds if and only if

$$
\sum_{i=1}^{l} \alpha_{i} \leq \sum_{i=1}^{l} \beta_{i} \text { for all } 1 \leq l<n
$$

and $\sum_{i=1}^{n} \alpha_{i}=\sum_{i=1}^{n} \beta_{i}$. If all inequalities in the above equation hold strictly and $\sum_{i=1}^{n} \alpha_{i}=\sum_{i=1}^{n} \beta_{i}$, then we say that $\psi$ is strictly majorized by $\varphi$. Majorization is an interesting and well-developed topic in linear algebra. For more details, we refer the reader to [7] and [8].

Nielsen's theorem establishes a connection between the theory of majorization and entanglement transformation. It is of fundamental importance in studying entanglement transformation and has many interesting corollaries. For example, by taking limits the asymptotic result of Bennett et al can be recovered from Nielsen's theorem. Unlike the transformations in the asymptotic regime, a direct implication of Nielsen's theorem is that the amount of entanglement decreases during the deterministic transformation. Let $E(|\psi\rangle)=-\sum_{i=1}^{n} \alpha_{i} \log _{2} \alpha_{i}$ be the entropy of entanglement of $|\psi\rangle$. Then by the properties 
of majorization, it follows that $|\psi\rangle \rightarrow|\varphi\rangle$ implies $E(|\varphi\rangle) \leq$ $E(|\psi\rangle)$ [6]. Indeed, these properties of majorization imply that any well-behaved entanglement measures, such as Renyi's entropy, or any other suitable concave functions, also decrease under LOCC. Intuitively, this means that a certain amount of entanglement will be lost in a LOCC transformation. It would be desirable to save some entanglement lost and reduce the net loss of entanglement in the transformation, since the saved entanglement can be used, for example, to increase the classical capacity of a quantum channel [9].

The possibility of recovering lost entanglement was first observed by Morikoshi [10]. We outline Morikoshi's recovering scheme as follows. Suppose Alice and Bob share an entangled state $|\psi\rangle$ and they can transform it into $|\varphi\rangle$ by LOCC. As we mentioned above, this process is generally a dissipative one in the sense that the quantity of entanglement in the target state is less than that in the source state. Suppose now an auxiliary state $|\chi\rangle$ is supplied to Alice and Bob. Instead of transforming $|\psi\rangle$ into $|\varphi\rangle$ directly, they perform collective operations on the joint state $|\psi\rangle \otimes|\chi\rangle$, and transform it into another joint state $|\varphi\rangle \otimes|\omega\rangle$. Of course, as required by Nielsen's theorem, entropy of entanglement of the whole system decreases too. But by choosing a suitable auxiliary state $|\chi\rangle$, sometimes a state $|\omega\rangle$ with more entropy of entanglement can be obtained. Intuitively, this process enables part of entanglement lost in the original transformation to be transferred to the auxiliary state, and it was termed partial entanglement recovery. Morikoshi demonstrated that partial entanglement recovery for a transformation between $2 \times 2$ states is always possible by using a $2 \times 2$ auxiliary state.

Partial entanglement recovery for transformations between higher dimensional states was considered by Bandyopadhyay et al. in [12]. To avoid trivial cases (a perfect recovery can always be achieved by letting $|\chi\rangle=|\varphi\rangle$ and $|\omega\rangle=|\psi\rangle$ ), a notion of genuine partial recovery was introduced. A partial recovery scheme is genuine if the dimension of the auxiliary state is smaller than that of the original source state. Then it was proven that for any states $|\psi\rangle$ and $|\varphi\rangle$ such that $\psi$ is strictly majorized by $\varphi$ and $n>2$, a genuine partial recovery is always possible by using only $2 \times 2$-dimensional auxiliary states. This extensively generalized the result in [10]. The possibility of genuine partial recovery for the transformation of $|\psi\rangle$ to $|\varphi\rangle$ such that $\psi$ is not strictly majorized by $\varphi$ was also examined carefully in [12]. However, several fundamental problems concerning partial entanglement recovery are still open. For example, the existence of genuine partial recovery in the case of $\alpha_{n}=\beta_{n}$ is unknown. Furthermore, the proof confirming the existence of auxiliary states for partial entanglement recovery presented in [12] is nonconstructive. In general, this proof method does not provide a way to find these auxiliary states efficiently.

In this paper, we study the feasibility of partial entanglement recovery. We consider the problem of whether a given entangled state can be used to recover some entanglement lost in a specified transformation. Our motivations are twofold. The first one is more theoretical. In some sense, the process of partial entanglement recovery reveals a new kind of application of quantum entanglement: it can be used to store some entan- glement lost in information processing tasks. So it is of great theoretical interest to characterize the entanglement recovering ability of a given entangled state, as it may lead to a better understanding of some fundamental properties of quantum entanglement. In addition, as will see later, the solution of the above problem leads us to a rich mathematical structure and provides new insight into the process of partial entanglement recovery. The second motivation is more practical. Suppose we are required to perform a couple of different entanglement transformations. In most applications the available entangled states shared between two parties are pre-specified and very limited. A solution to the above problem will help us to determine whether partial entanglement recovery for these transformations is possible with other pre-specified entangled states. It is also worth noting that this problem is more general than the ones discussed in [10] and [12], and its solution automatically resolves many trivial cases.

To state the above problem more formally, let us assume that $|\psi\rangle$ and $|\varphi\rangle$ are the source state and the target state of the specified transformation, respectively, and let $|\chi\rangle$ be the given auxiliary state. Furthermore, suppose that $|\psi\rangle$ can be transformed into $|\varphi\rangle$ with certainty using LOCC. Our goal is to determine whether there exists another state $|\omega\rangle$ satisfying (i) the transformation of $|\psi\rangle \otimes|\chi\rangle$ to $|\varphi\rangle \otimes|\omega\rangle$ can be implemented with certainty using LOCC, and (ii) $|\omega\rangle$ is more entangled than $|\chi\rangle$. Next, we clarify a subtle point: what is meant by the statement that a state is more entangled than another one? An exact mathematical definition is needed. One way to do this is to use some measures of entanglement such as entropy of entanglement mentioned above, as in [10] and [12]. Note that for deterministic transformations, a single measure of entanglement is usually not enough to quantify entanglement amount since there exist incomparable states $|\psi\rangle$ and $|\varphi\rangle$, i.e., neither $|\psi\rangle \rightarrow|\varphi\rangle$ nor $|\varphi\rangle \rightarrow|\psi\rangle$ is possible [6]. So in the present paper, we adopt an alternative view-point: we say that $|\omega\rangle$ is more entangled than $|\chi\rangle$ if $|\omega\rangle \rightarrow|\chi\rangle$ and $|\chi\rangle \nrightarrow|\omega\rangle$. By Nielsen's theorem, this is equivalent to $\omega \prec \chi$ and $\chi \neq \omega$ (here both $\chi$ and $\omega$ are in non-increasing order). We believe that this view-point is more reasonable than only considering a single measure. Now the mathematical problem of the feasibility of partial entanglement recovery can be clearly formulated as follows:

Problem 1. Given a triple of states $(|\psi\rangle,|\varphi\rangle,|\chi\rangle)$ such that $\psi \prec \varphi$, determine whether there exists a state $|\omega\rangle$ such that $\psi \otimes \chi \prec \varphi \otimes \omega, \omega \prec \chi$, and $\chi \neq \omega$.

If such a state $|\omega\rangle$ does exist, then we call it a solution of Problem 1. In the above formulation we made no additional assumptions on the dimension of $\chi$ except that it is finite. So even in the case that the dimension of $\chi$ is larger than or equal to that of $\psi$ (and $\varphi$ ), the above problem still makes sense. This enables us to consider the process of partial entanglement recovery in a general mathematical framework. It is also worth pointing out that Problem 1 cannot be directly solved by linear programming methods because the majorization relation $\psi \otimes$ $\chi \prec \varphi \otimes \omega$ cannot be expressed by linear constraints, unless we know how to order a tensor product of two probability vectors, $\varphi \otimes \omega$. The main difficulty here is that the order of 
$\varphi \otimes \omega$ is not related in any simple way to the orders of $\varphi$ and $\omega$.

The principal aim of the present paper is to solve Problem 1 stated above. We first introduce three indices of uniformity for bipartite entangled pure states. With the aid of these indices, we prove that whether $|\chi\rangle$ can save some entanglement lost for the transformation of $|\psi\rangle$ to $|\varphi\rangle$ only depends on the target state $|\varphi\rangle$ and the presence of the equalities in the majorization relation $\psi \prec \varphi$. To be concise, let $|\psi\rangle$ be a state with $m$ distinct Schmidt coefficients, say, $\alpha_{1}^{\prime}>\cdots>\alpha_{m}^{\prime} \geq 0$. If $m>1$, then the maximal local uniformity of $|\psi\rangle$, denoted by $L_{u}(|\psi\rangle)$, is given by the maximal ratio of $\alpha_{i+1}^{\prime}$ and $\alpha_{i}^{\prime}$ for all $1 \leq i \leq m-1$. In contrast, the global uniformity of $|\psi\rangle$, denoted by $g_{u}(|\psi\rangle)$, is given by the ratio of $\alpha_{m}^{\prime}$ and $\alpha_{1}^{\prime}$. In the special case of $m=1$, both indices are defined to be 1 . These indices have many useful properties. Indeed, they are key tools in studying partial entanglement recovery. With these notions, Problem 1 is completely solved in the case where $\psi$ is strictly majorized by $\varphi$ (Theorem 3.2). We achieve this goal by considering two cases. First, Problem 1 is examined carefully for a special case where all nonzero Schmidt coefficients of $|\chi\rangle$ are identical, i.e., $L_{u}(|\chi\rangle)=0$ or $L_{u}(|\chi\rangle)=1$. Second we consider the general case that $0<L_{u}(|\chi\rangle)<1$ and prove:

(1) if $L_{u}(|\chi\rangle)>g_{u}(|\varphi\rangle)$, then $|\chi\rangle$ can recover some entanglement lost for the transformation of $|\psi\rangle$ to $|\varphi\rangle$;

(2) if $L_{u}(|\chi\rangle)=g_{u}(|\varphi\rangle)$, then there is only a special form of $|\varphi\rangle$ for which $|\chi\rangle$ can save some entanglement lost in the transformation of $|\psi\rangle$ to $|\varphi\rangle$; and

(3) if $L_{u}(|\chi\rangle)<g_{u}(|\varphi\rangle)$, then $|\chi\rangle$ cannot recover entanglement lost in any transformation with the target $|\varphi\rangle$.

It should be pointed out that the proof we present provides an explicit construction of the resulting state $|\omega\rangle$. In view of this, the above results are very useful in pursuing practical applications of partial entanglement recovery. Some interesting special cases of these results are also discussed.

For the case where $\psi$ is not strictly majorized by $\varphi$, a complete solution of Problem 1 appears to be very difficult. Nevertheless, two sufficient conditions for partial entanglement recovery are presented (Theorems 4.1 and 4.2). Employing these conditions as tools we show that the genuine partial recovery is not always possible when the dimension of the target state is larger than $2 \times 2$. For example, if $n=3$ and $\alpha_{1}=\beta_{1},|\chi\rangle$ should be at least a $3 \times 3$ entangled state, which means any recovery scheme cannot be genuine. (This result in fact has been obtained implicitly in [12]). When $\alpha_{1}=\beta_{1}$ and $\alpha_{n}=\beta_{n}$, we show that $4 \times 4$-dimensional auxiliary states are necessary and sufficient. In the case where $n=4$, a genuine partial recovery is not possible. On the other hand, even in these special cases, it still makes sense to consider whether $|\chi\rangle$ is useful in recovering entanglement lost in the transformation of $|\psi\rangle$ to $|\varphi\rangle$.

Besides the mathematical characterization of partial entanglement recovery outlined above, we also present an algorithmic approach to Problem 1. Let $n$ and $k$ be the dimensions of $\psi$ (as well as $\varphi$ ) and $\chi$ (as well as $\omega$ ), respectively. Our goal now is to design polynomial time algorithms in $n$ or/and $k$ to solve Problem 1. As mentioned above, the main difficulty in solving Problem 1 lies in the fact that the order of the tensor product $\varphi \otimes \omega$ cannot be determined by a simple method even after we know the orders of $\varphi$ and $\omega$. Thus one cannot apply standard linear programming techniques directly. A naive enumeration of the possible orders of $\varphi \otimes \omega$ yields about $(n k)$ ! results, which is intractable. A simple but powerful lemma is introduced to reduce the number of orders of the tensor product. The basic idea behind this lemma comes from the observation that for a fixed $\varphi, \varphi \otimes \omega$ has at most $O\left((k n)^{2(k-1)}\right)$ different orders when $\omega$ varies. This number of the possible orders is only a polynomial in $n$ when $k$ is treated as a constant. For each possible order, we can employ linear programming methods to solve the majorization inequality $\psi \otimes \chi \prec \varphi \otimes \omega$. Consequently, an algorithm of time complexity $O\left(n^{2 k-1} \log _{2} n\right)$ is obtained (Theorem 5.1). This algorithm is not efficient in the case where $k$ can vary freely. Fortunately, by examining the mathematical structure of partial entanglement recovery carefully, we can further refine this algorithm into a new one with time complexity $O\left(n^{2} k^{4}\right)$ (Theorem 5.2). Therefore we can efficiently determine the feasibility of partial entanglement recovery by using algorithmic methods.

To illustrate the utility of the above results, we show that partial entanglement recovery also happens in situations such as quantum catalysis, mutual catalysis, and multiplecopy transformation. As an interesting application, we consider the generation of maximally entangled states using the scheme of partial entanglement recovery. We prove that any transformation with the Schmidt coefficient vector of the source state being strictly majorized by that of the target state can always concentrate some partially entangled state into a maximally entangled one. We also find a close connection between partial entanglement recovery and quantum catalysis (see [18], [19], [21]). That is, if a transformation can be implemented with certainty by using some quantum catalyst, then entanglement lost in the transformation can be partially recovered by a suitable auxiliary state. Moreover, we show that partial entanglement recovery is directly connected to mutual catalysis [23]. As a consequence, a systematic construction of the instances with mutual catalysis effect is sketched. When we consider the possibility of partial entanglement recovery in multiple-copy transformations (see [15], [19], and [21]), we notice a very interesting phenomenon: although an auxiliary state cannot be used to do partial entanglement recovery for a single-copy transformation, it can recover some entanglement lost in certain multiple-copy transformations.

The rest of the paper is organized as follows. Section II presents some notations and concepts, including the definitions of uniformity indices. In Section III, we present a complete solution to Problem 1 in the case that $\psi$ is strictly majorized by $\varphi$. We consider general transformations in Section IV and give two sufficient conditions for partial entanglement recovery. Some special but interesting cases of these conditions are investigated in detail. In Section $\mathrm{V}$ we discuss the feasibility of partial entanglement recovery from an algorithmic viewpoint and present two algorithms to solve Problem 1. To understand whether partial entanglement recovery is possible in situations such as quantum catalysis, mutual catalysis, and multiplecopy transformation, we give more examples and discussions in Section VI. In Section VII, we draw a brief conclusion. 
The proofs of some lemmas and theorems are completed in Appendices.

\section{PRELIMINARIES}

First, it is helpful to introduce some notations associated with finite dimensional vectors. Let $x$ be an $n$-dimensional vector. The dimension of $x$ is denoted by $\operatorname{dim}(x)$, i.e., $\operatorname{dim}(x)=n$. The notation $x^{\downarrow}$ is used to stand for the vector that is obtained by rearranging the components of $x$ into non-increasing order. Similarly, $x^{\uparrow}$ denotes the vector that is obtained by rearranging the components of $x$ into nondecreasing order. The notation $x^{\oplus k}$ denotes the direct sum of $x$ with itself $k$ many times. In particular, for constant $c, c^{\oplus k}$ is the $k$-dimensional vector $(c, c, \ldots, c)$. If every component of $x$ is nonnegative, then we can write

$$
x^{\downarrow}=\left(x_{1}^{\prime \oplus k_{1}}, \ldots, x_{m}^{\prime \oplus k_{m}}\right),
$$

where $x_{1}^{\prime}>\cdots>x_{m}^{\prime} \geq 0, k_{i} \geq 1$ for each $i=1, \ldots, m$, and $\sum_{i=1}^{m} k_{i}=n$. The above form of $x^{\downarrow}$ is usually called the compact form of $x$. It is obvious that the compact form of a nonnegative vector is unique when the dimension of the vector space under consideration is fixed.

The sum of the $m$ largest components of the vector $x$ is denoted by $e_{m}(x)$. That is, $e_{m}(x)=\sum_{i=1}^{m} x_{i}^{\downarrow}$. It is easily to verify that $e_{m}(x)$ is a continuous function of $x$ for each $m=1, \ldots, n$.

We say that $x$ is majorized by $y$, denoted by $x \prec y$, if

$$
e_{m}(x) \leq e_{m}(y), \text { for all } 1 \leq m \leq n-1
$$

and $e_{n}(x)=e_{n}(y)$. If all inequalities in Eq. (1) are strict and $e_{n}(x)=e_{n}(y)$, then we follow the terminology in [12] and say that $x$ is strictly majorized by $y$, denoted by $x \triangleleft y$.

A vector $x$ is a segment of a vector $y$ if there exist $i \geq 1$ and $k \geq 0$ such that $x=\left(y_{i}, y_{i+1}, \ldots, y_{i+k}\right)$.

Now we apply the above terminology to bipartite entangled pure states. Let $|\psi\rangle$ be an $n \times n$ entangled pure state with ordered Schmidt coefficients $\alpha_{1} \geq \alpha_{2} \geq \cdots \geq \alpha_{n} \geq 0$. As we have mentioned in the introduction, the symbol $\psi$ is used to denote the Schmidt coefficient vector of $|\psi\rangle$, i.e., $\psi=\left(\alpha_{1}, \ldots, \alpha_{n}\right)$, which is just an $n$-dimensional probability vector. We often identify the compact form of $\psi$ with the compact form of state $|\psi\rangle$. We call $|\psi\rangle$ an $n \times n$ maximally entangled state if the compact form of $|\psi\rangle$ reduces to $\left(\frac{1}{n}\right)^{\oplus n}$; otherwise we say that $|\psi\rangle$ is a partially entangled state. If $\varphi^{\prime \downarrow}$ is a segment of $\varphi^{\downarrow}$, then we call $\left|\varphi^{\prime}\right\rangle$ an unnormalized state.

To apply Nielsen's theorem to unnormalized states, it can be restated as: $|\psi\rangle \rightarrow|\varphi\rangle$ if and only if $\psi \prec \varphi$.

We define $S(|\varphi\rangle)$ to be the set of all $n \times n$ entangled pure states $|\psi\rangle$ which can be directly transformed into $|\varphi\rangle$ by LOCC. By Nielsen's theorem, $S(|\varphi\rangle)=\{|\psi\rangle: \psi \prec \varphi\}$. We also define $S^{o}(|\varphi\rangle)$ to be the set of all $n \times n$ states $|\psi\rangle$ such that $\psi$ is strictly majorized by $\varphi$, i.e., $S^{o}(|\varphi\rangle)=$ $\{|\psi\rangle: \psi \triangleleft \varphi\}$. It should be noted that states $|\psi\rangle$ in $S(|\varphi\rangle)$ are required to have the same dimension as $|\varphi\rangle$. Such a requirement forces us to distinguish $S(|\varphi\rangle)$ from $S\left(\left|\varphi^{\prime}\right\rangle\right)$ when $|\varphi\rangle$ and $\left|\varphi^{\prime}\right\rangle$ are essentially the same state but their dimensions are different. For example, let $\varphi=(0.5,0.25,0.25)$ and $\varphi^{\prime}=(0.5,0.25,0.25,0)$. It is obvious that the states $|\varphi\rangle$ and $\left|\varphi^{\prime}\right\rangle$ are essentially the same. However, according to the above definitions, $S(|\varphi\rangle)$ is completely different from $S\left(\left|\varphi^{\prime}\right\rangle\right)$. This design decision in defining $S(|\varphi\rangle)$ enables us to considerably simplify the presentation of our main results. The same remark also applies to the definition of $S^{o}(|\varphi\rangle)$.

In this paper, the phrase 'bipartite entangled pure state' is used frequently. So, for convenience, sometimes we abbreviate it to 'state' or 'quantum state'. This should not cause any confusion from the context.

Now we introduce three notions which are key mathematical tools in describing partial entanglement recovery.

Definition 2.1: Let $|\psi\rangle$ be an $n \times n$ partially entangled state with compact form $\left(\alpha_{1}^{\prime \oplus k_{1}}, \ldots, \alpha_{m}^{\prime \oplus k_{m}}\right)$, where $n=\sum_{i=1}^{m} k_{i}$ and $m>1$. Then

(i) the minimal local uniformity of $|\psi\rangle$ is defined by

$$
l_{u}(|\psi\rangle)=\min \left\{\frac{\alpha_{i+1}^{\prime}}{\alpha_{i}^{\prime}}: 1 \leq i<m\right\} ;
$$

(ii) the maximal local uniformity of $|\psi\rangle$ is defined by

$$
L_{u}(|\psi\rangle)=\max \left\{\frac{\alpha_{i+1}^{\prime}}{\alpha_{i}^{\prime}}: 1 \leq i<m\right\} ;
$$

(iii) the global uniformity of $|\psi\rangle$ is defined by

$$
g_{u}(|\psi\rangle)=\frac{\alpha_{m}^{\prime}}{\alpha_{1}^{\prime}} .
$$

It is easy to see that the minimal local uniformity, the maximal local uniformity, and the global uniformity of a quantum state $|\psi\rangle$ with $\psi^{\downarrow}=\left(\alpha_{1}, \ldots, \alpha_{n}\right)$ may be rewritten in a slightly different way:

$$
\begin{gathered}
l_{u}(|\psi\rangle)=\min \left\{\frac{\alpha_{i+1}}{\alpha_{i}}: 1 \leq i<n\right\} \\
L_{u}(|\psi\rangle)=\max \left\{\frac{\alpha_{i+1}}{\alpha_{i}}: 1 \leq i<n \text { and } \alpha_{i}>\alpha_{i+1}\right\} ; \\
g_{u}(|\psi\rangle)=\frac{\alpha_{n}}{\alpha_{1}} .
\end{gathered}
$$

The above rewriting will help us to simplify some proofs.

From the above rewriting of Definition 2.1 it is easy to see that both $l_{u}(|\psi\rangle)$ and $g_{u}(|\psi\rangle)$ are continuous with respect to $|\psi\rangle$. Thus it is reasonable to define the minimal local uniformity and the global uniformity of a maximally entangled state to be 1 . However, such a continuous property does not hold for the maximal local uniformity. To keep many properties of these indices valid even in the case that the quantum state under consideration is maximally entangled, it is convenient to define the maximal local uniformity of a maximally entangled state to be 1 . Also, for the sake of convenience, when the dimension of the state under consideration is one-dimensional, we define the uniform indices as 1 .

In applying the above definitions of uniformity indices, it should be noted that the dimension of $|\psi\rangle$ is somewhat arbitrary, as one can append zeroes to the vector $\psi$ and thereby increase its dimension without changing the underlying quantum state. Suppose that the number of nonzero components of $\psi$ is $n$. If $|\psi\rangle$ is treated as an $n \times n$ state, all the above three uniformity indices are positive. However, if we append zeroes 
to $\psi$ and yield a state $\left|\psi^{\prime}\right\rangle$, then the uniformity indices of $\left|\psi^{\prime}\right\rangle$ are changed rapidly. For example, let $\psi=(0.5,0.25,0.25)$ and $\psi^{\prime}=(0.5,0.25,0.25,0)$. It is obvious that both the minimal local uniformity and the global uniformity of $|\psi\rangle$ are 0.5 . However, the minimal local uniformity and the global uniformity of $\left|\psi^{\prime}\right\rangle$ are changed into 0 . To avoid any confusion that may be caused by the phenomenon that we just mentioned in the above definition, the dimension of the states are treated as fixed. In other words, if $\left|\psi^{\prime}\right\rangle$ is obtained from $|\psi\rangle$ by appending zeros in its Schmidt coefficient vector, they may be thought of being two different states. Therefore, it is reasonable to allow that sometimes $l_{u}(|\psi\rangle) \neq l_{u}\left(\left|\psi^{\prime}\right\rangle\right.$ ) (as well as $L_{u}(|\psi\rangle) \neq L_{u}\left(\left|\psi^{\prime}\right\rangle\right)$ and $g_{u}(|\psi\rangle) \neq g_{u}\left(\left|\psi^{\prime}\right\rangle\right)$ ).

Some simple but useful properties of the three indices defined above are presented in the following:

Lemma 2.1: Let $|\psi\rangle$ and $|\varphi\rangle$ be two quantum states with compact forms $\psi^{\downarrow}=\left(\alpha_{1}^{\prime \oplus k_{1}}, \ldots, \alpha_{r}^{\prime \oplus k_{r}}\right)$ and $\varphi^{\downarrow}=$ $\left(\beta_{1}^{\prime \oplus l_{1}}, \ldots, \beta_{s}^{\prime \oplus l_{s}}\right)$, respectively. Then

(1) $0 \leq l_{u}(|\psi\rangle), L_{u}(|\psi\rangle), g_{u}(|\psi\rangle) \leq 1$.

(2) $l_{u}^{r-1}(|\psi\rangle) \leq g_{u}(|\psi\rangle) \leq l_{u}(|\psi\rangle)$.

(3) $g_{u}(|\psi\rangle) \leq L_{u}^{r-1}(|\psi\rangle)$.

(4) $g_{u}(|\psi\rangle) \leq l_{u}(|\psi\rangle) \leq L_{u}(|\psi\rangle)$.

(5) if $r=s$ and $\alpha_{i}^{\prime}=\beta_{i}^{\prime}$ for $i=1, \ldots, r$, then $l_{u}(|\psi\rangle)=l_{u}(|\varphi\rangle)$. Similarly, $L_{u}(|\psi\rangle)=L_{u}(|\varphi\rangle)$ and $g_{u}(|\psi\rangle)=g_{u}(|\varphi\rangle)$.

(6) if $|\psi\rangle \rightarrow|\varphi\rangle$, then $g_{u}(|\psi\rangle) \geq g_{u}(|\varphi\rangle)$.

Proof. (1)-(5) follow immediately from Definition 2.1. (6) follows directly from Definition 2.1 and the fact that if $|\psi\rangle \rightarrow|\varphi\rangle$ then $\alpha_{1}^{\prime} \leq \beta_{1}^{\prime}$ and $\alpha_{r}^{\prime} \geq \beta_{s}^{\prime}$.

We give some remarks on the above properties. (1) shows that the three indices of minimal local uniformity, maximal local uniformity and global uniformity are all between 0 and 1 . Moreover, they take value 1 if the state is maximally entangled. The minimal local uniformity and the global uniformity take the value 0 if the state in question has zero as one Schmidt coefficient, while the maximal local uniformity takes value 0 if it is a maximally entangled state in a state space with lower dimension, i.e., with a compact form $\left(\left(\frac{1}{m}\right)^{\oplus m}, 0^{\oplus n-m}\right)$ for some $m<n$. If $l_{u}(|\psi\rangle)=0$ or $l_{u}(|\psi\rangle)=1$, i.e., $|\psi\rangle$ has zero as a Schmidt coefficient or it is maximally entangled, then both the inequalities in (2) hold with equalities. In the case that $0<l_{u}(|\psi\rangle)<1$, the first equality in (2) holds if the distinct Schmidt coefficients of $|\psi\rangle$ form a geometric sequence; while the second equality holds if $|\psi\rangle$ has at most two distinct Schmidt coefficients. The equality in (3) holds if and only if the distinct Schmidt coefficients of $|\psi\rangle$ form a geometric sequence. (4) can be analyzed similarly. (5) means that these indices only depend on distinct Schmidt coefficients of the state. (6) indicates that global uniformity is decreasing under LOCC.

In addition to these trivial properties displayed in Lemma 2.1. the following lemma presents three more interesting properties of global uniformity and minimal local uniformity:

Lemma 2.2: Let $|\psi\rangle$ and $|\varphi\rangle$ be two quantum states. Then

(1) $g_{u}(|\psi\rangle \otimes|\varphi\rangle)=g_{u}(|\psi\rangle) g_{u}(|\varphi\rangle)$. In particular, $g_{u}\left(|\psi\rangle^{\otimes m}\right)=g_{u}^{m}(|\psi\rangle)$ for any $m \geq 1$.

(2) $l_{u}(|\psi\rangle \otimes|\varphi\rangle) \geq \min \left\{l_{u}(|\psi\rangle), l_{u}(|\varphi\rangle)\right\}$.
(3) $l_{u}(|\psi\rangle) \leq l_{u}\left(|\psi\rangle^{\otimes k}\right) \leq \min \left\{\frac{\alpha_{2}^{\prime}}{\alpha_{1}^{\prime}}, \frac{\alpha_{r}^{\prime}}{\alpha_{r-1}^{\prime}}\right\}$, where $\psi^{\downarrow}=$ $\left(\alpha_{1}^{\prime \oplus k_{1}}, \ldots, \alpha_{r}^{\prime \oplus k_{r}}\right)$.

Proof. (1) follows immediately by Definition 2.1. (3) is a simple application of (2) and Definition 2.1. So it is enough to prove (2).

Let $\psi^{\downarrow}=\left(\alpha_{1}, \cdots, \alpha_{m}\right)$ and $\varphi^{\downarrow}=\left(\beta_{1}, \cdots, \beta_{n}\right)$. Since the minimal uniformity $l_{u}$ is a continuous functional, we can assume without loss of generality that all components of $\psi$ and $\varphi$ are positive. Let

$$
a=\alpha_{p} \beta_{q} \text { and } b=\alpha_{r} \beta_{s}, \quad a \leq b,
$$

be any two successive elements of the ordered probability vector $(\psi \otimes \varphi)^{\downarrow}$. It is obvious that $r<m$ or $s<n$. Suppose $r<m$ is the case, let us try to prove

$$
l_{u}(|\psi\rangle) \leq \frac{a}{b} .
$$

Indeed, from the definition of $l_{u}$ we have

$$
\begin{aligned}
l_{u}(|\psi\rangle) & \leq \frac{\alpha_{r+1}}{\alpha_{r}} \\
& =\frac{\alpha_{r+1} \beta_{s}}{\alpha_{r} \beta_{s}} \leq 1 .
\end{aligned}
$$

Thus $\alpha_{r+1} \beta_{s} \leq b$. But, since $a$ and $b$ are successive elements, $\alpha_{r+1} \beta_{s}$ cannot belong to the interval $(a, b)$, that is,

$$
\alpha_{r+1} \beta_{s} \leq a .
$$

From Eqs. (3) and (4) we get immediately Eq. (2).

If $r=m$ then we can be sure $s<n$. Thus we can apply analogous arguments to prove that

$$
l_{u}(|\varphi\rangle) \leq \frac{a}{b}
$$

In both cases one has

$$
\min \left\{l_{u}(|\psi\rangle), l_{u}(|\varphi\rangle)\right\} \leq \frac{a}{b} .
$$

Since this is true for any successive $a \leq b$, we have proved statement (2).

The above lemma deserves some more remarks. Intuitively, (1) shows that the global uniformity is multiplicative under tensor product. (2) means that the tensor product of two states is at least as uniform as one of them. (3) provides an upper bound and a lower bound respectively for the minimal local uniformity of any state consisting of multiple copies of a given state. More interestingly, it shows that the minimal local uniformity of a $2 \times 2$ or $3 \times 3$ state remains invariant under tensor products involving multiple copies.

One of the most interesting applications of the uniformity indices introduced above is that they provide a characterization of when a strict majorization relation holds.

Lemma 2.3: Let $|\varphi\rangle$ and $|\chi\rangle$ be two quantum states, and let $S^{o}(|\varphi\rangle) \otimes|\chi\rangle$ denote the set of all states of the form $|\psi\rangle \otimes|\chi\rangle$ with $|\psi\rangle$ in $S^{o}(|\varphi\rangle)$, i.e., $S^{o}(|\varphi\rangle) \otimes|\chi\rangle=\{|\psi\rangle \otimes|\chi\rangle:|\psi\rangle \in$ $\left.S^{o}(|\varphi\rangle)\right\}$. Then

$$
S^{o}(|\varphi\rangle) \otimes|\chi\rangle \subseteq S^{o}(|\varphi\rangle \otimes|\chi\rangle) \Leftrightarrow l_{u}(|\chi\rangle)>g_{u}(|\varphi\rangle) .
$$

Proof. See Appendix A.

Roughly speaking, the above lemma shows that if the auxiliary state $|\chi\rangle$ is uniform enough, then the strict majorization 
relation $\psi \otimes \chi \triangleleft \varphi \otimes \chi$ can be kept providing $\psi \triangleleft \varphi$, and vice versa. What we would like to emphasize here is that the only constraint on the source state $|\psi\rangle$ is $\psi \triangleleft \varphi$.

In the introduction we have frequently used the notion of partial entanglement recovery. We present a rigorous definition as follows.

Definition 2.2: Let $|\psi\rangle$ and $|\varphi\rangle$ be two $n \times n$ states, and let $|\chi\rangle$ be a $k \times k$ state. We say that $|\chi\rangle$ can do partial entanglement recovery for the transformation of $|\psi\rangle$ to $|\varphi\rangle$ if there exists a $k \times k$ state $|\omega\rangle$ such that

(i) both the transformations of $|\psi\rangle \otimes|\chi\rangle$ to $|\varphi\rangle \otimes|\omega\rangle$ and $|\omega\rangle$ to $|\chi\rangle$ can be realized with certainty under LOCC. That is, $|\psi\rangle \otimes|\chi\rangle \rightarrow|\varphi\rangle \otimes|\omega\rangle$ and $|\omega\rangle \rightarrow|\chi\rangle$;

(ii) the transformation of $|\chi\rangle$ to $|\omega\rangle$ cannot be achieved with certainty under LOCC. That is, $|\chi\rangle \nrightarrow|\omega\rangle$.

Some remarks follow:

1) In the above definition, both the dimensions of the source state $|\psi\rangle$ and the target state $|\varphi\rangle$ are $n \times n$. Similarly, the dimensions of the auxiliary state $|\chi\rangle$ and the resulting state $|\omega\rangle$ are both $k \times k$. These constraints are reasonable since during the transformation process the state space under consideration isn't modified. Intuitively, $|\psi\rangle$ and $|\varphi\rangle$ are two different states of the same two particles entangled between Alice and Bob. The dimensions of these particles are assumed to be finite and fixed. Similar arguments apply to the states $|\chi\rangle$ and $|\omega\rangle$. This is in fact the reason that we have to require that the dimension $|\varphi\rangle$ is fixed in defining $S(|\varphi\rangle), S^{o}(|\varphi\rangle), l_{u}(|\varphi\rangle), L_{u}(|\varphi\rangle)$, and $g_{u}(|\varphi\rangle)$, since all of them are introduced in this paper to describe partial entanglement recovery.

2) According to Nielsen's theorem, the above definition can be rewritten as: a $k \times k$ auxiliary state $|\chi\rangle$ can do partial entanglement recovery for a transformation of $|\psi\rangle$ to $|\varphi\rangle$ if there exists another $k \times k$ state $|\omega\rangle$ such that all three relations $\psi \otimes \chi \prec \varphi \otimes \omega, \omega \prec \chi$, and $\chi^{\downarrow} \neq \omega^{\downarrow}$ hold simultaneously.

$3)$ It is obvious that $(x, 0) \prec(y, 0)$ if and only if $x \prec$ $y$. Without loss of generality, we can assume that the number of nonzero Schmidt coefficients of $|\psi\rangle$ is $n$. In other words, all Schmidt coefficients of the source state are positive.

For technical simplicity, we apply the above discussions not only to normalized but also to unnormalized states. Sometimes we shall use a clause such as ' $|\chi\rangle$ can save some entanglement lost for the transformation of $|\psi\rangle$ to $|\varphi\rangle$ ', and we shall say that $|\psi\rangle$ can transfer some entanglement into the state $|\chi\rangle$ whenever $|\chi\rangle$ can do partial entanglement recovery for the transformation from $|\psi\rangle$ to some unspecified target state $|\varphi\rangle$ to mean that ' $|\chi\rangle$ can do partial entanglement recovery for the transformation of $|\psi\rangle$ to $|\varphi\rangle$ '.

\section{PARTial ENTANglement ReCOVERY FOR A TRANSFORMATION BETWEEN STATES WITH STRICT MAJORIZATION}

In this section, we focus on whether a given auxiliary state $|\chi\rangle$ can do partial entanglement recovery for a transformation of $|\psi\rangle$ to $|\varphi\rangle$ such that $|\psi\rangle$ is in $S^{o}(|\varphi\rangle)$. A necessary and sufficient condition for such a recovery is presented. Thus, a complete characterization of such auxiliary states $|\chi\rangle$ is obtained.

First, we define the distance between $|\psi\rangle$ and $|\varphi\rangle$ to be the Euclidean distance between two $n$-dimensional ordered probability vectors $\psi^{\downarrow}$ and $\varphi^{\downarrow}$, i.e,

$$
\||\psi\rangle-|\varphi\rangle \|=\sqrt{\sum_{i=1}^{n}\left(\psi_{i}^{\downarrow}-\varphi_{i}^{\downarrow}\right)^{2}} .
$$

Before presenting the main result of this section, we prove a useful theorem. Assuming that $|\psi\rangle$ is in $S^{o}(|\varphi\rangle)$, we shall prove that if $l_{u}(|\chi\rangle)>g_{u}(|\varphi\rangle)$ then a suitable collective operation can transform the joint state $|\psi\rangle \otimes|\chi\rangle$ into another joint state $|\varphi\rangle \otimes|\omega\rangle$ such that $|\omega\rangle$ is not 'far from' $|\chi\rangle$. Surprisingly, this result does not depend on which source state $|\psi\rangle$ we have chosen at the beginning.

Theorem 3.1: Let $|\psi\rangle$ and $|\varphi\rangle$ be two states with $|\psi\rangle \in$ $S^{o}(|\varphi\rangle)$. If $|\chi\rangle$ is an auxiliary state such that $l_{u}(|\chi\rangle)>$ $g_{u}(|\varphi\rangle)$, then there exists a positive number $\delta$ such that for any state $|\omega\rangle$ satisfying $\||\omega\rangle-|\chi\rangle \|<\delta$, it holds that

$$
|\psi\rangle \otimes|\chi\rangle \rightarrow|\varphi\rangle \otimes|\omega\rangle .
$$

Proof. Since $l_{u}(|\chi\rangle)>g_{u}(|\varphi\rangle)$ and $|\psi\rangle \in S^{o}(|\varphi\rangle)$, it follows from Lemma 2.3 that

$$
\psi \otimes \chi \triangleleft \varphi \otimes \chi .
$$

Notice that a small enough perturbation on the right hand side of Eq. (6) will not change the relation ' $\triangleleft$ ' since every inequality in Eq. (1) is strict. Thus it is possible to take a sufficiently small positive number $\delta$ such that for any state $|\omega\rangle$ satisfying $\||\omega\rangle-|\chi\rangle \|<\delta$, the relation $\psi \otimes \chi \prec \varphi \otimes \omega$ holds, which confirms the validity of Eq. (5). With that we complete the proof of Theorem 3.1

The following simple corollary of Theorem 3.1 establishes a connection between uniformity indices and partial entanglement recovery.

Corollary 3.1: If $g_{u}(|\varphi\rangle)<l_{u}(|\chi\rangle)<1$, then $|\chi\rangle$ can do partial entanglement recovery for any transformation of $|\psi\rangle$ to $|\varphi\rangle$ with $|\psi\rangle \in S^{o}(|\varphi\rangle)$.

Intuitively, if the minimal local uniformity of a partially entangled pure state $|\chi\rangle$ is larger than the global uniformity of $|\varphi\rangle$, then the transformation of $|\psi\rangle$ to $|\varphi\rangle$ such that $\psi \triangleleft \varphi$ can always increase the entanglement degree of $|\chi\rangle$.

Example 3.1: Let $|\psi\rangle$ and $|\varphi\rangle$ be two $2 \times 2$ states with $\psi^{\downarrow}=(a, 1-a)$ and $\varphi^{\downarrow}=(b, 1-b)$, where $\frac{1}{2}<a<b<1$. The goal here is to find a $2 \times 2$ state that can do partial entanglement recovery for the transformation of $|\psi\rangle$ to $|\varphi\rangle$.

Take an auxiliary state $|\chi(p)\rangle$ with $\chi^{\downarrow}(p)=(p, 1-p)$. It is easy to check that $|\psi\rangle$ is in $S^{o}(|\varphi\rangle)$. By Corollary 3.1, if $|\chi(p)\rangle$ satisfies

$$
g_{u}(|\varphi\rangle)<l_{u}(|\chi(p)\rangle)<1
$$

then $|\chi(p)\rangle$ can be used to do partial entanglement recovery for the transformation $|\psi\rangle \rightarrow|\varphi\rangle$. It is easy to see that Eq. (7) 
is equivalent to

$$
\frac{1-b}{b}<\frac{1-p}{p}<1
$$

or $\frac{1}{2}<p<b$. The desired state $|\omega\rangle$ such that both $|\psi\rangle \otimes|\chi\rangle \rightarrow|\varphi\rangle \otimes|\omega\rangle$ and $|\omega\rangle \rightarrow|\chi\rangle$ hold can be taken as $|\omega\rangle=|\chi(p-\epsilon)\rangle$ with a suitably small positive number $\epsilon$. It is obvious that $|\chi\rangle \nrightarrow|\omega\rangle$ whenever $\epsilon$ is positive but small enough.

In Example 3.1, the condition of $p<b$ means that $|\chi(p)\rangle$ is more entangled than $|\varphi\rangle$. A simple analysis shows that this condition is also necessary to guarantee that $|\chi(p)\rangle$ does partial entanglement recovery for the transformation of $|\psi\rangle$ to $|\varphi\rangle$. So we rediscover the main result in [10]: for $2 \times 2$ dimensional states, the auxiliary state $|\chi\rangle$ can do nontrivial partial entanglement recovery for a transformation with target state $|\varphi\rangle$ if and only if $|\chi\rangle$ is more entangled than $|\varphi\rangle$.

Example 3.2: This is a generalization of Example 3.1 Let $|\psi\rangle$ and $|\varphi\rangle$ be two states such that $|\psi\rangle$ is in $S^{o}(|\varphi\rangle)$. Our aim here is to decide whether there exists some $2 \times 2$ state that can do partial entanglement recovery for the transformation of $|\psi\rangle$ to $|\varphi\rangle$.

Take an auxiliary state $|\chi(p)\rangle$ with $\chi^{\downarrow}(p)=(p, 1-p)$. By Corollary 3.1, if

$$
g_{u}(|\varphi\rangle)<l_{u}(|\chi(p)\rangle)<1
$$

then $|\chi(p)\rangle$ can do partial entanglement recovery for the transformation of $|\psi\rangle$ to $|\varphi\rangle$. Moreover, Eq. (8) is equivalent to

$$
\frac{1}{2}<p<\frac{1}{1+g_{u}(|\varphi\rangle)} .
$$

Therefore, the entanglement lost in the transformation of $|\psi\rangle$ to $|\varphi\rangle$ can always be partially recovered by a $2 \times 2$ state $|\chi(p)\rangle$ satisfying Eq. (9). Again, the desired state $|\omega\rangle$ such that both $|\psi\rangle \otimes|\chi\rangle \rightarrow|\varphi\rangle \otimes|\omega\rangle$ and $|\omega\rangle \rightarrow|\chi\rangle$ can be taken as $|\omega\rangle=|\chi(p-\epsilon)\rangle$ with a suitably small positive number $\epsilon$.

In Example 3.2, we show that the entanglement lost in a transformation of $|\psi\rangle$ to $|\varphi\rangle$ such that $|\psi\rangle \in S^{o}(|\varphi\rangle)$ can always be partially recovered by a $2 \times 2$ state $|\chi\rangle$, and the explicit construction of such a state $|\chi\rangle$ is also presented. This is a considerable refinement of Theorem 1 in [12]. We also point out that in the proof of Theorem 1 in [12], an important constraint on $p$, i.e., $p \beta_{n}<(1-p) \beta_{1}$ or $l_{u}(|\chi(p)\rangle)>g_{u}(|\varphi\rangle)$, is missing, therefore the case (ii) in the proof of Theorem 1 in [12] is possible if $x=s=n$ and $y=t=0$, which makes the proof there invalid.

Corollary 3.1 only provides us with a sufficient condition for which $|\chi\rangle$ can be used to receive some entanglement lost in a transformation of $|\psi\rangle$ to $|\varphi\rangle$ with $|\psi\rangle \in S^{o}(|\varphi\rangle)$. However, this condition is too strong to be satisfied in many cases. Nevertheless, the following theorem gives a weaker condition, and indeed it provides a complete characterization of states $|\chi\rangle$ that can be used to do partial entanglement recovery for a transformation with target state $|\varphi\rangle$ and source state $|\psi\rangle$ in $S^{o}(|\varphi\rangle)$.

Theorem 3.2: Let $|\psi\rangle$ and $|\varphi\rangle$ be two $n \times n$ states such that $|\psi\rangle$ is in $S^{o}(|\varphi\rangle)$, and let $|\chi\rangle$ be a $k \times k$ auxiliary state. Then $|\chi\rangle$ can do partial entanglement recovery for the transformation of $|\psi\rangle$ to $|\varphi\rangle$ if and only if one of the following three cases holds:

(i) $\quad L_{u}(|\chi\rangle)=0$ and $n a \geq n^{\prime}(a+1)$. Here $a$ and $n^{\prime}$ are the numbers of nonzero Schmidt coefficients of $|\chi\rangle$ and $|\varphi\rangle$, respectively;

(ii) $g_{u}(|\varphi\rangle)<L_{u}(|\chi\rangle)<1$;

(iii) $L_{u}(|\chi\rangle)=g_{u}(|\varphi\rangle)$ and $\varphi^{\downarrow}=\left(\chi^{\prime \oplus m} / C\right)^{\downarrow}$. Here $\chi^{\prime}$ is a segment of $\chi \downarrow$ with only two distinct components, $C$ is a normalization factor, and $m \geq 1$.

Moreover, if none of the above cases holds, then $|\chi\rangle$ cannot do partial entanglement recovery for any transformation of $|\psi\rangle$ to $|\varphi\rangle$ such that $|\psi\rangle$ is in $S(|\varphi\rangle)$.

Proof. See Appendix B.

To better understand the above theorem, we give the following remarks:

1) The case that $L_{u}(|\chi\rangle)=1$ is not included in cases (i), (ii), and (iii). Hence a simple corollary of Theorem 3.2 is that a maximally entangled state cannot be used to do partial entanglement recovery. This is reasonable since for such a state $|\chi\rangle$, there does not exist a $k \times k$ state $|\omega\rangle$ which is more entangled than $|\chi\rangle$.

2) The case that $L_{u}(|\chi\rangle)=0$ is slightly different from the above case and is more interesting. Although $|\chi\rangle$ is a maximally entangled state in a state space of lower dimension $a \times a$ with $a<k$, it is only partially entangled when it is considered as a $k \times k$ state. Hence it is still possible to transform $|\psi\rangle \otimes|\chi\rangle$ into another state $|\varphi\rangle \otimes|\omega\rangle$, where $|\omega\rangle$ is more entangled than $|\chi\rangle$. Case (i) shows that the necessary and sufficient condition is that the Schmidt numbers of $|\chi\rangle$ and $|\varphi\rangle$ satisfy a simple inequality $n a \geq n^{\prime}(a+1)$. In some sense, the solution in this case explains why the dimensions of the states need to be fixed.

3) Case (ii) means that if $|\chi\rangle$ is partially entangled and the maximal local uniformity of $|\chi\rangle$ is larger than the global uniformity of $|\varphi\rangle$, then $|\chi\rangle$ can be used to save some entanglement lost in the transformation of $|\psi\rangle$ to $|\varphi\rangle$. This case provides a feasible sufficient condition for partial entanglement recovery.

4) Case (iii) is of special interest. It supplies the solution at the critical point $L_{u}(|\chi\rangle)=g_{u}(|\varphi\rangle)$. As we will see, the proof of this case is very complicated. We include this case for the following two reasons. First, from the aspect of the completeness of the solution. Including such a special case enables us to completely solve the feasibility of partial entanglement recovery for all $\psi$ and $\varphi$ with $\psi \triangleleft \varphi$. Second, from the special form that $|\varphi\rangle$ should satisfy. A careful observation shows that $|\varphi\rangle$ has only two different Schmidt coefficients and should be constructed by repeating a segment of $\chi^{\downarrow}$ finitely many times. In our opinion this provides new insight into the process of partial entanglement recovery. In addition, in the proof of this case we have extensively employed the techniques introduced in the present paper and the properties of majorization. Hopefully, these proof techniques will be useful in solving other problems 
in quantum entanglement theory.

In sum, Theorem 3.2 provides a necessary and sufficient condition under which $|\chi\rangle$ can do partial entanglement recovery for some transformation with the target state $|\varphi\rangle$. Therefore it can be treated as a basic result about partial entanglement recovery. In view of Theorem 3.2 it seems reasonable to use maximal local uniformity to describe the partial entanglement recovery power of an auxiliary state.

It is worth noting that in the above theorem, the choice of $|\psi\rangle$ has some free degree. That is, if $|\chi\rangle$ can be used to do partial entanglement recovery for a transformation of $|\psi\rangle$ to $|\varphi\rangle$ such that $|\psi\rangle$ is in $S^{o}(|\varphi\rangle)$, then for any $\left|\psi^{\prime}\right\rangle \in S^{o}(|\varphi\rangle)$, $|\chi\rangle$ can also recover entanglement lost in the transformation of $\left|\psi^{\prime}\right\rangle$ to $|\varphi\rangle$.

Theorem 3.2 has many interesting corollaries. We only consider the following one where the auxiliary state $|\chi\rangle$ is $2 \times 2$-dimensional.

Corollary 3.2: If $|\chi\rangle$ and $|\varphi\rangle$ are two partially entangled states with $\chi^{\downarrow}=(p, 1-p)$ and $\varphi^{\downarrow}=\left(\beta_{1}, \ldots, \beta_{n}\right)$, then $|\chi\rangle$ can be used to do partial entanglement recovery for the transformation of $|\psi\rangle$ to $|\varphi\rangle$ such that $|\psi\rangle$ is in $S^{o}(|\varphi\rangle)$ if and only if one of the following three cases holds:

(i) $p=1$ and $n \geq 2 n^{\prime}$, where $n^{\prime}$ is the number of nonzero components of $\varphi$;

(ii) $\frac{1}{2}<p<\frac{\beta_{1}}{\beta_{1}+\beta_{n}}$;

(iii) $p=\frac{\beta_{1}}{\beta_{1}+\beta_{n}}$ and $|\varphi\rangle$ has a special form such that $\varphi^{\downarrow}=$ $\left((p, 1-p)^{\oplus k} / k\right)^{\downarrow}$ for some $k \geq 1$.

Moreover, if none of (i)-(iii) is satisfied, then $|\chi\rangle$ cannot do partial entanglement recovery for any transformation of $|\psi\rangle$ to $|\varphi\rangle$ such that $|\psi\rangle$ is in $S(|\varphi\rangle)$.

The most interesting part of the Corollary 3.2 is case (iii). The following example demonstrates this point.

Example 3.3: Let $|\chi\rangle,\left|\varphi^{\prime}\right\rangle,\left|\varphi^{\prime \prime}\right\rangle$, and $\left|\varphi^{\prime \prime \prime}\right\rangle$ be four states with $\chi^{\downarrow}=(p, 1-p), \varphi^{\prime \downarrow}=(p, p, 1-p, 1-p) / 2, \varphi^{\prime \prime \downarrow}=$ $(p, p, 1-p) /(1+p)$, and $\varphi^{\prime \prime \prime} \downarrow=(p, 1-p, 1-p) /(2-p)$, where $\frac{1}{2}<p<1$. Obviously,

$$
L_{u}(|\chi\rangle)=g_{u}\left(\left|\varphi^{\prime}\right\rangle\right)=g_{u}\left(\left|\varphi^{\prime \prime}\right\rangle\right)=g_{u}\left(\left|\varphi^{\prime \prime \prime}\right\rangle\right)=\frac{1-p}{p} .
$$

By Corollary 3.2, it is easy to see that $|\chi\rangle$ can do partial entanglement recovery for any transformation of $|\psi\rangle$ to $\left|\varphi^{\prime}\right\rangle$ with $|\psi\rangle \in S^{o}\left(\left|\varphi^{\prime}\right\rangle\right)$ since $\varphi^{\downarrow}=\left((p, 1-p)^{\oplus 2} / 2\right)^{\downarrow}$.

However, again by the above corollary, $|\chi\rangle$ cannot recover anything for any transformations with the target states $\left|\varphi^{\prime \prime}\right\rangle$ or $\left|\varphi^{\prime \prime \prime}\right\rangle$.

Until now we only deal with the transformations of $|\psi\rangle$ to $|\varphi\rangle$ such that $\psi$ is strictly majorized by $\varphi$. What about the other cases? In next section, we will prove two more general theorems about partial entanglement recovery where $|\psi\rangle$ and $|\varphi\rangle$ only need to satisfy the non-strict majorization relation $\psi \prec \varphi$.

\section{PARTIAL ENTANGLEMENT RECOVERY FOR A GENERAL TRANSFORMATION}

In this section we deal with partial entanglement recovery for a class of more general transformations. Before proceeding to the main results, it will be helpful to introduce some notations. Let $x$ and $y$ be two finite dimensional vectors. We write $x \sqsubset y$ or $y \sqsupset x$ if $x_{1}^{\downarrow}<y_{1}^{\downarrow}$ and $x_{1}^{\uparrow}>y_{1}^{\uparrow}$. Roughly speaking, $x \sqsubset y$ means that the values of the extreme components of $x$ are strictly bounded by those of $y$. We use the formal expression $\frac{x^{\prime}}{x^{\prime \prime}} \sqsupset \frac{y^{\prime}}{y^{\prime \prime}}$ as a convenient rewriting of $x^{\prime} \otimes y^{\prime \prime} \sqsupset x^{\prime \prime} \otimes y^{\prime}$.

For simplicity, in this section we only deal with vectors that are already in non-increasing order. That is, for a finite dimensional vector $x$, we assume that $x=x^{\downarrow}$.

We now introduce the following concept.

Definition 4.1: A decomposition of a vector $x$ is a sequence of vectors $x^{1}, \ldots, x^{m}$ satisfying

(i) each of these vectors has dimension at least one, i.e., $\operatorname{dim}\left(x^{i}\right) \geq 1$; and

(ii) $x$ is the direct sum of these vectors, i.e., $x=$ $\left(x^{1}, \ldots, x^{m}\right)$, or simply, $x=\oplus_{i=1}^{m} x^{m}$.

The following simple lemma provides a special decomposition of two vectors $x$ and $y$ such that $x \prec y$.

Lemma 4.1: If $x$ and $y$ are vectors satisfying $x \prec y$, then $x$ and $y$ can be uniquely decomposed as $x=\left(x^{1}, \ldots, x^{m}\right)$ and $y=\left(y^{1}, \ldots, y^{m}\right)$ such that

(i) $x^{i} \triangleleft y^{i}$ or $x^{i}=y^{i}$ for each $i=1, \ldots, m$; and

(ii) there does not exist an index $i$ such that $x^{i}=y^{i}$ and $x^{i+1}=y^{i+1}$ hold simultaneously.

Proof. The proof is simple, and the details are omitted.

The decompositions of $x$ and $y$ in Lemma 4.1 are called the normal decompositions of $x$ and $y$.

Motivated by Lemma 4.1 we shall define two index sets $I_{x, y}$ and $D_{x, y}$ for any vectors $x$ and $y$ satisfying $x \prec y$. Suppose that $x$ and $y$ have normal decompositions as in Lemma 4.1. Then we define

$$
I_{x, y}=\left\{i: x^{i}=y^{i} \text { and } 1 \leq i \leq m\right\}
$$

and

$$
D_{x, y}=\left\{i: x^{i} \triangleleft y^{i} \text { and } 1 \leq i \leq m\right\} .
$$

It is obvious that

$$
I_{x, y} \cap D_{x, y}=\emptyset \text { and } I_{x, y} \cup D_{x, y}=\{1, \ldots, m\} .
$$

One can easily check that $x \triangleleft y$ is equivalent to $I_{x, y}=\emptyset$ and $D_{x, y}=\{1\}$.

In what follows, we only consider the auxiliary state with positive Schmidt coefficients, as our major purpose here is to find the states that can do partial entanglement recovery for a given transformation. For simplicity, the maximally entangled state is also not considered.

With these preliminaries, we present one of the main results in this section, which gives a sufficient condition under which $|\chi\rangle$ can do partial entanglement recovery for a transformation of $|\psi\rangle$ to $|\varphi\rangle$.

Theorem 4.1: Let $|\psi\rangle$ and $|\varphi\rangle$ be two states with normal decompositions $\psi=\left(\psi^{1}, \ldots, \psi^{m}\right)$ and $\varphi=\left(\varphi^{1}, \ldots, \varphi^{m}\right)$ such that $\psi$ is majorized by $\varphi$, and let $|\chi\rangle$ be an auxiliary state with a similar decomposition to $|\psi\rangle$ and $|\varphi\rangle$, say, $\chi=$ $\left(\chi^{1}, \ldots, \chi^{m}\right)$. If

$$
\frac{\chi^{i}}{\chi^{j}} \sqsupset \frac{\varphi^{i}}{\varphi^{j}} \text {, for all } i \in I_{\psi, \varphi} \text { and } j \in D_{\psi, \varphi}
$$


and

$$
\min \left\{l_{u}\left(\left|\chi^{i}\right\rangle\right): 1 \leq i \leq m\right\}>\max \left\{g_{u}\left(\left|\varphi^{i}\right\rangle\right): i \in D_{\psi, \varphi}\right\},
$$

then $|\chi\rangle$ can do partial entanglement recovery for the transformation of $|\psi\rangle$ to $|\varphi\rangle$.

Moreover, if $|\chi\rangle$ satisfies Eqs. (10) and (11), then there exists a positive number $\delta$ such that for any state $|\omega\rangle$ with a decomposition $\omega=\left(\omega^{1}, \ldots, \omega^{m}\right)$ satisfying

(i) $\omega^{i}=\chi^{i}$ for any $i \in I_{\psi, \varphi}$; and

(ii) $\left\|\omega^{i}-\chi^{i}\right\|<\delta$ and $\sum \omega^{i}=\sum \chi^{i}$ for any $i \in D_{\psi, \varphi}$, the transformation of $|\psi\rangle \otimes|\chi\rangle$ to $|\varphi\rangle \otimes|\omega\rangle$ can be realized with certainty by LOCC, i.e., $|\psi\rangle \otimes|\chi\rangle \rightarrow|\varphi\rangle \otimes|\omega\rangle$.

Proof. See Appendix C.

Here we give some remarks:

1) In the above theorem whether $|\chi\rangle$ can save entanglement lost for the transformation of $|\psi\rangle$ to $|\varphi\rangle$ does not directly depend on the choice of the source state $|\psi\rangle$. It only depends on the decomposition of the target state $|\varphi\rangle$ and the index sets $I_{\psi, \varphi}$ and $D_{\psi, \varphi}$. For this reason, in what follows, it is not necessary to specify the source state $|\psi\rangle$ clearly. We only need to give a decomposition of $\varphi$ and two index sets $I$ and $D$. Based on these conditions, we can identify a class of auxiliary states $|\chi\rangle$ that can do partial entanglement recovery for any transformation of $|\psi\rangle$ to $|\varphi\rangle$ with source state $|\psi\rangle$ satisfying $I_{\psi, \varphi}=I$ and $D_{\psi, \varphi}=D$.

2) When $|\chi\rangle$ can be used to do partial entanglement recovery for the transformation of $|\psi\rangle$ to $|\varphi\rangle$, the more entangled state $|\omega\rangle$ generated from $|\chi\rangle$ is also explicitly given by the above theorem. It should be noted that in general the resulting state $|\omega\rangle$ is determined by the states $|\chi\rangle,|\psi\rangle$ and $|\varphi\rangle$ together, although the choice of $|\chi\rangle$ doesn't depend on the source state $|\psi\rangle$. In other words, sometimes there may not exist a universal state $|\omega\rangle$ in the sense that $\left|\psi^{\prime}\right\rangle \otimes|\chi\rangle \rightarrow|\varphi\rangle \otimes|\omega\rangle,|\omega\rangle \rightarrow|\chi\rangle$ and $|\chi\rangle \nrightarrow|\omega\rangle$ hold for all states $\left|\psi^{\prime}\right\rangle$ with $D_{\psi^{\prime}, \varphi}=D_{\psi, \varphi}$ and $I_{\psi^{\prime}, \varphi}=I_{\psi, \varphi}$

We now examine some special cases of Theorem 4.1 The first special case is that both $D$ and $I$ are singletons.

Corollary 4.1: Let $|\varphi\rangle$ and $|\chi\rangle$ be two states with decompositions $\varphi=\left(\varphi^{1}, \varphi^{2}\right)$ and $\chi=\left(\chi^{1}, \chi^{2}\right)$, and let $I=\{1\}$ and $D=\{2\}$. If $|\varphi\rangle$ and $|\chi\rangle$ satisfy

$$
\frac{\chi^{1}}{\chi^{2}} \sqsupset \frac{\varphi^{1}}{\varphi^{2}}
$$

and

$$
\min \left\{l_{u}\left(\left|\chi^{1}\right\rangle\right), l_{u}\left(\left|\chi^{2}\right\rangle\right)\right\}>g_{u}\left(\left|\varphi^{2}\right\rangle\right)
$$

then $|\chi\rangle$ can do partial entanglement recovery for any transformation of $|\psi\rangle$ to $|\varphi\rangle$ with source state $|\psi\rangle$ such that $I_{\psi, \varphi}=I$ and $D_{\psi, \varphi}=D$.

A corresponding result for the dual of case $I=\{2\}$ and $D=\{1\}$ can be obtained by exchanging $\chi^{1}$ with $\chi^{2}$ and $\varphi^{1}$ with $\varphi^{2}$ in Eq. (12) and Eq. (13), respectively.

For the sake of convenience, for two $n$-dimensional vectors $x$ and $y$ with $x \prec y$, we define $\Delta_{x, y}$ as the set of all indices $m$ such that the inequality in Eq. (1) holds with an equality, i.e.,

$$
\Delta_{x, y}=\left\{m: e_{m}(x)=e_{m}(y) \text { and } 1 \leq m \leq n-1\right\} .
$$

Note that $1 \in \Delta_{x, y}$ is equivalent to $x_{1}=y_{1}$ and $n-1 \in \Delta_{\psi, \varphi}$ is equivalent to $x_{n}=y_{n}$.

Now we present two examples to illustrate the use of Corollary 4.1

Example 4.1: Let $|\psi\rangle$ and $|\varphi\rangle$ be two $n \times n$ states such that $|\psi\rangle$ is in $S(|\varphi\rangle)(n>2)$. Assume that $\Delta_{\psi, \varphi}=\{1\}$. We hope to find an auxiliary state $|\chi\rangle$ with the minimal dimension to do partial entanglement recovery for the transformation of $|\psi\rangle$ to $|\varphi\rangle$.

To be more specific, let $\varphi=\left(\beta_{1}, \ldots, \beta_{n}\right)$. Since $\Delta_{\psi, \varphi}=$ $\{1\}$, it is obvious that $\varphi$ has a normal decomposition $\varphi=$ $\left(\varphi^{1}, \varphi^{2}\right)$, where $\varphi^{1}=\left(\beta_{1}\right)$ and $\varphi^{2}=\left(\beta_{2}, \ldots, \beta_{n}\right)$. Moreover, $I_{\psi, \varphi}=\{1\}$ and $D_{\psi, \varphi}=\{2\}$. Take an auxiliary state $|\chi\rangle$ with $\chi=\left(\chi^{1}, \chi^{2}\right)$, where $\chi^{1}=\left(\gamma_{1}\right), \chi^{2}=\left(\gamma_{2}, \gamma_{3}\right)$, and $\gamma_{1}>\gamma_{2}>\gamma_{3}>0$.

By Corollary 4.1, if $|\chi\rangle$ satisfies Eqs. 12]-(13), then $|\chi\rangle$ can do partial entanglement recovery for the transformation of $|\psi\rangle$ to $|\varphi\rangle$. So we have

$$
\begin{gathered}
\gamma_{1} / \gamma_{2}>\beta_{1} / \beta_{2}, \\
\gamma_{1} / \gamma_{3}<\beta_{1} / \beta_{n},
\end{gathered}
$$

and

$$
\gamma_{3} / \gamma_{2}>\beta_{n} / \beta_{2}
$$

By Eqs. (14) and (16), we can take positive numbers $\lambda$ and $\mu$ such that

$$
\gamma_{1}=\gamma_{2}(1+\lambda) \beta_{1} / \beta_{2}
$$

and

$$
\gamma_{3}=\gamma_{2}(1+\mu) \beta_{n} / \beta_{2} .
$$

Substituting Eqs. (17) and (18) into Eq. (15) yields $0<\lambda<$ $\mu$. Moreover, the constraint $\gamma_{2}>\gamma_{3}$ and Eq. (18) yield $\mu<$ $\left(\beta_{2}-\beta_{n}\right) / \beta_{n} \cdot \gamma_{2}$ is used to make the following normalization condition satisfied:

$$
\sum_{i=1}^{3} \gamma_{i}=1 .
$$

Notice that $\beta_{2}>\beta_{n}$. One can easily check that such $\left(\gamma_{1}, \gamma_{2}, \gamma_{3}\right)$ satisfying Eqs. (17)-19) is a solution of the system of inequalities defined by Eqs. (14)-116). The parameters $\lambda$ and $\mu$ satisfy $0<\lambda<\mu<\left(\beta_{2}-\beta_{n}\right) / \beta_{n}$.

Thus by Corollary 4.1 the $3 \times 3$ auxiliary state $|\chi\rangle$ can do partial entanglement recovery for the transformation of $|\psi\rangle$ to $|\varphi\rangle$. Moreover, the state $|\omega\rangle$ such that both $|\psi\rangle \otimes|\chi\rangle \rightarrow|\varphi\rangle \otimes|\omega\rangle$ and $|\omega\rangle \rightarrow|\chi\rangle$ hold can be chosen as $\omega=\left(\gamma_{1}, \gamma_{2}-\epsilon, \gamma_{3}+\epsilon\right)$ with a sufficiently small positive number $\epsilon$.

We point out that the existence of such an auxiliary state $|\chi\rangle$ with $\chi=\left(\gamma_{1}, \gamma_{2}, \gamma_{3}\right)$ has been proven in Theorem 3 in [12], where $|\chi\rangle$ is of the form $\chi(p, q)=(p, q, 1-p-q)$, $p \geq q \geq 1-p-q \geq 0$. However, an important constraint on the $p$ and $q$ or the region $R$, i.e., $(1-p-q) \beta_{2}>q \beta_{n}$, is missing in [12]. Thus an additional case which is not included 
in Case (i) and Case (ii) in [12] is possible, which makes that proof invalid.

A dual case of Example 4.1 is as follows:

Example 4.2: Let $|\psi\rangle$ and $|\varphi\rangle$ be two $n \times n$ states such that $|\psi\rangle$ is in $S(|\varphi\rangle)(n>2)$. Assume that $\Delta_{\psi, \varphi}=\{n-1\}$. We hope to find an auxiliary state $|\chi\rangle$ with the minimal dimension to do partial entanglement recovery for the transformation of $|\psi\rangle$ to $|\varphi\rangle$.

To be more specific, let $\varphi=\left(\beta_{1}, \ldots, \beta_{n}\right)$. Since $\Delta_{\psi, \varphi}=$ $\{n-1\}$, it is easy to check that $\varphi$ has a normal decomposition $\varphi=\left(\varphi^{1}, \varphi^{2}\right)$, where $\varphi^{1}=\left(\beta_{1}, \ldots, \beta_{n-1}\right)$ and $\varphi^{2}=\left(\beta_{n}\right)$. Similarly, let an auxiliary sate $|\chi\rangle$ have a decomposition $\chi=$ $\left(\chi^{1}, \chi^{2}\right)$, where $\chi^{1}=\left(\gamma_{1}, \gamma_{2}\right), \chi^{2}=\left(\gamma_{3}\right)$, and $\gamma_{1}>\gamma_{2}>$ $\gamma_{3}>0$. By Eqs. (12) and (13) again, noticing that $I_{\psi, \varphi}=\{2\}$ and $D_{\psi, \varphi}=\{1\}$, we have the following system of inequalities:

$$
\begin{gathered}
\gamma_{3} / \gamma_{1}>\beta_{n} / \beta_{1}, \\
\gamma_{3} / \gamma_{2}<\beta_{n} / \beta_{n-1},
\end{gathered}
$$

and

$$
\gamma_{2} / \gamma_{1}>\beta_{n-1} / \beta_{1}
$$

By using a similar argument as in Example 4.1 we can take

$$
\gamma_{2}=\gamma_{1}(1+\mu) \beta_{n-1} / \beta_{1}
$$

and

$$
\gamma_{3}=\gamma_{1}(1+\lambda) \beta_{n} / \beta_{1},
$$

where $0<\lambda<\mu<\left(\beta_{1}-\beta_{n-1}\right) / \beta_{n-1} .(\lambda<\mu$ is deduced by substituting Eqs. (23) and (24) into Eq. (21), $\mu<\left(\beta_{1}-\right.$ $\left.\beta_{n-1}\right) / \beta_{n-1}$ comes from Eq. (23) and $\left.\gamma_{1}>\gamma_{2}\right) \cdot \gamma_{1}$ is taken to validate the following normalization condition

$$
\sum_{i=1}^{3} \gamma_{i}=1 .
$$

Since $\beta_{1}>\beta_{n-1}$, one can easily check that such a state $|\chi\rangle$ with $\chi=\left(\gamma_{1}, \gamma_{2}, \gamma_{3}\right)$ is a solution of the inequalities system defined by Eqs. (20)-(22). Thus by Corollary $4.1|\chi\rangle$ can do partial entanglement recovery for the transformation of $|\psi\rangle$ to $|\varphi\rangle$. Again, the desired state $|\omega\rangle$ such that both $|\psi\rangle \otimes|\chi\rangle \rightarrow|\varphi\rangle \otimes|\omega\rangle$ and $|\omega\rangle \rightarrow|\chi\rangle$ hold can be chosen as $\omega=\left(\gamma_{1}-\epsilon, \gamma_{2}+\epsilon, \gamma_{3}\right)$ with a suitably small positive number $\epsilon$.

If one of the cases $\Delta_{\psi, \varphi}=\{1\}$ or $\Delta_{\psi, \varphi}=\{n-1\}$ occurs, we can always use a $3 \times 3$ state $|\chi\rangle$ to partially recover entanglement lost in the transformation of $|\psi\rangle$ to $|\varphi\rangle$. The explicit construction of such $|\chi\rangle$ has also been presented in the above examples.

The following corollary is another important special case of Theorem 4.1 .

Corollary 4.2: Let $|\varphi\rangle$ and $|\chi\rangle$ be two states with $\varphi=$ $\left(\varphi^{1}, \varphi^{2}, \varphi^{3}\right)$ and $\chi=\left(\chi^{1}, \chi^{2}, \chi^{3}\right) . I=\{1,3\}$ and $D=\{2\}$. If $|\chi\rangle$ and $|\varphi\rangle$ satisfy

$$
\begin{aligned}
& \frac{\chi^{1}}{\chi^{2}} \sqsupset \frac{\varphi^{1}}{\varphi^{2}}, \\
& \frac{\chi^{3}}{\chi^{2}} \sqsupset \frac{\varphi^{3}}{\varphi^{2}},
\end{aligned}
$$

and

$$
\min \left\{l_{u}\left(\left|\chi^{1}\right\rangle\right), l_{u}\left(\left|\chi^{2}\right\rangle\right), l_{u}\left(\left|\chi^{3}\right\rangle\right)\right\}>g_{u}\left(\left|\varphi^{2}\right\rangle\right),
$$

then $|\chi\rangle$ can do partial entanglement recovery for any transformation of $|\psi\rangle$ to $|\varphi\rangle$ with $I_{\psi, \varphi}=I$ and $D_{\psi, \varphi}=D$.

A very interesting application of the above corollary is the following:

Example 4.3: Let $|\psi\rangle$ and $|\varphi\rangle$ be two $n \times n$ states such that $|\psi\rangle$ is in $S(|\varphi\rangle)(n>3)$. Assume $\Delta_{\psi, \varphi}=\{1, n-1\}$. Our purpose here is to find an auxiliary state to do partial entanglement recovery for the transformation of $|\psi\rangle$ to $|\varphi\rangle$.

Take a $4 \times 4$ auxiliary state $|\chi\rangle$ with $\chi=\left(\gamma_{1}, \gamma_{2}, \gamma_{3}, \gamma_{4}\right)$. Let us decompose $\varphi$ and $\chi$, respectively, into $\varphi=\left(\varphi^{1}, \varphi^{2}, \varphi^{3}\right)$ and $\chi=\left(\chi^{1}, \chi^{2}, \chi^{3}\right)$, where $\varphi^{1}=\left(\beta_{1}\right), \varphi^{2}=\left(\beta_{2}, \ldots, \beta_{n-1}\right)$, $\varphi^{3}=\left(\beta_{n}\right), \chi^{1}=\left(\gamma_{1}\right), \chi^{2}=\left(\gamma_{2}, \gamma_{3}\right), \chi^{3}=\left(\gamma_{4}\right)$, and $\gamma_{1}>\gamma_{2}>\gamma_{3}>\gamma_{4}>0$. Since $\Delta_{\psi, \varphi}=\{1, n-1\}$, it is easy to check that $I_{\psi, \varphi}=\{1,3\}$ and $D_{\psi, \varphi}=\{2\}$. Thus by Corollary 4.2. $|\chi\rangle$ can do partial entanglement recovery for the transformation of $|\psi\rangle$ to $|\varphi\rangle$ if Eqs. (26)-(28) hold. A routine calculation leads to the following solution of Eqs. (26)-228:

$$
\begin{gathered}
\gamma_{1}=\gamma_{2}(1+\lambda) \beta_{1} / \beta_{2}, \\
\gamma_{3}=\gamma_{2}(1+\eta)(1+\mu) \beta_{n-1} / \beta_{2}, \\
\gamma_{4}=\gamma_{2}(1+\mu) \beta_{n} / \beta_{2},
\end{gathered}
$$

where $1+\lambda<(1+\eta)(1+\mu)<\beta_{2} / \beta_{n-1}, \lambda, \mu$, and $\eta$ are all positive real numbers, and $\gamma_{2}$ is used to validate the normalization condition

$$
\sum_{i=1}^{4} \gamma_{i}=1 .
$$

So such an auxiliary state $|\chi\rangle$ for partial entanglement recovery always exists.

Ultimately, to partially recover entanglement lost in the transformation of $|\psi\rangle$ to $|\varphi\rangle$, it is sufficient to use an auxiliary state $|\chi\rangle$ with dimension $4 \times 4$. Again, the more entangled state $|\omega\rangle$ generated from $|\chi\rangle$ after the recovery process can be chosen as $\omega=\left(\gamma_{1}, \gamma_{2}-\epsilon, \gamma_{3}+\epsilon, \gamma_{4}\right)$, where $\epsilon$ is a sufficiently small positive number.

In [12], it is proven that any $3 \times 3$ state cannot be used to partially recover entanglement lost in the transformation of $|\psi\rangle$ to $|\varphi\rangle$ with $\psi \prec \varphi$ and $\Delta_{\psi, \varphi}=\{1, n-1\}$. By the above example, we are able to show that $4 \times 4$ auxiliary states are necessary and sufficient to do partial entanglement recovery for this special case.

In practice, we hope that the dimension of the auxiliary state $|\chi\rangle$ is as small as possible. In Theorem 4.1, if there are two successive integers $i$ and $i+1$ both contained in $D_{\psi, \varphi}$ (in $I_{\psi, \varphi}$ this case cannot happen), we in fact can combine $\chi^{i}$ with $\chi^{i+1}$ to reduce the dimension of $\chi$. So a careful investigation of the structure of $D_{\psi, \varphi}$ is necessary.

Let us see a simple example. Suppose that for states $|\psi\rangle$ and $|\varphi\rangle, I_{\psi, \varphi}=\{1,4,7,12\}$ and $D_{\psi, \varphi}=\{2,3,5,6,8,9,10,11\}$. By the construction in Theorem 4.1, we should use an auxiliary state $|\chi\rangle$ with $\chi=\left(\chi^{1}, \ldots, \chi^{12}\right)$, where each $\chi^{i}\left(i \in D_{\psi, \varphi}\right)$ has dimension at least 2 . Thus the vector 
$\chi$ has dimension at least $\left|I_{\psi, \varphi}\right|+2\left|D_{\psi, \varphi}\right|=20$. If we combine the successive integers in $D_{\psi, \varphi}$ together, we have $D_{\psi, \varphi}^{\prime}=\{\{2,3\},\{5,6\},\{8,9,10,11\}\}$, and the dimension of $\chi$ is reduced to $\left|I_{\psi, \varphi}\right|+2\left|D_{\psi, \varphi}^{\prime}\right|=10$.

More formally, suppose that $|\psi\rangle$ and $|\varphi\rangle$ are two states with normal decompositions $\psi=\left(\psi^{1}, \ldots, \psi^{m}\right)$ and $\varphi=$ $\left(\varphi^{1}, \ldots, \varphi^{m}\right)$. Let

$$
I_{\psi, \varphi}=\left\{k_{1}, \ldots, k_{p}\right\}, 1 \leq p \leq m,
$$

where

$$
0=k_{0}<k_{1}<\cdots<k_{p}<k_{p+1}=m+1 .
$$

We define

$$
D_{\psi, \varphi}^{\prime}=\left\{D_{i}: D_{i} \neq \emptyset \text { and } 0 \leq i \leq p\right\}
$$

where

$$
D_{i}=\left\{s: k_{i}+1 \leq s \leq k_{i+1}-1\right\}
$$

The constraint $D_{i} \neq \emptyset$ in the definition of $D_{\psi, \varphi}^{\prime}$ needs a careful explanation. For any $1 \leq i \leq p$, we have that $k_{i} \in I_{\psi, \varphi}$ implies $k_{i}+1 \notin I_{\psi, \varphi}$. So $D_{i} \neq \emptyset$ in this case. However, if $k_{1}=1$ or $k_{p}=m$ then we have $D_{0}=\emptyset$ or $D_{p}=\emptyset$, respectively. To avoid these two trivial cases, the constraint $D_{i} \neq \emptyset$ is necessary. In particular, if $I_{\psi, \varphi}=\emptyset$ then $D_{\psi, \varphi}^{\prime}=\left\{D_{\psi, \varphi}\right\}=$ $\{\{1, \ldots, m\}\}$. For the sake of convenience, we also define

$$
I_{\psi, \varphi}^{\prime}=\left\{\{i\}: i \in I_{\psi, \varphi}\right\} .
$$

In the following discussions, we shall use the elements of $I_{\psi, \varphi}^{\prime}$ and $D_{\psi, \varphi}^{\prime}$ as indices. We define the natural order of the elements in $I_{\psi, \varphi}^{\prime} \cup D_{\psi, \varphi}^{\prime}$ as

$$
D_{0}<\left\{k_{1}\right\}<D_{1}<\left\{k_{2}\right\}<\cdots<\left\{k_{p}\right\}<D_{p}
$$

where we assume that any term which doesn't exist should be omitted automatically without affecting the orders of other terms.

Suppose that $J$ is a finite set of integers. We use the notations $\max J$ and $\min J$ to denote the maximal and the minimal elements of $J$, respectively. For any real function $f($. defined on $J$, the expression arg $\min _{k \in J} f(k)$ denotes the index $i \in J$ such that $f(i)=\min _{k \in J} f(k)$ (here we assume that there is a unique $i$ of $J$ that can attain the minimum).

Now we can present another condition for the existence of partial entanglement recovery, which complements Theorem 4.1

Theorem 4.2: Let $|\psi\rangle$ and $|\varphi\rangle$ be two states with normal decompositions $\psi=\left(\psi^{1}, \ldots, \psi^{m}\right)$ and $\varphi=\left(\varphi^{1}, \ldots, \varphi^{m}\right)$ such that $\psi$ is majorized by $\varphi$, and let $|\chi\rangle$ be an auxiliary state with a decomposition $\chi=\left(\chi^{D_{0}}, \chi^{\left\{k_{1}\right\}}, \chi^{D_{1}}, \ldots, \chi^{\left\{k_{p}\right\}}, \chi^{D_{p}}\right)=$ $\oplus_{i \in I_{\psi, \varphi}^{\prime} \cup D_{\psi, \varphi}^{\prime}} \chi^{i}$. If

$$
\frac{\chi^{\{i\}}}{\chi^{J}} \sqsupset \frac{\varphi^{i}}{\varphi^{J_{i}}}
$$

for all $i \in I_{\psi, \varphi}, J \in D_{\psi, \varphi}^{\prime}$ and $J_{i}=\arg \min _{k \in J}|i-k|$,

$$
\min \left\{l_{u}\left(\left|\chi^{J}\right\rangle\right): J \in D_{\psi, \varphi}^{\prime}\right\}>\max \left\{g_{u}\left(\left|\varphi^{i}\right\rangle\right): i \in D_{\psi, \varphi}\right\}
$$

and

$$
\begin{aligned}
& \min \left\{l_{u}\left(\left|\chi^{\{i\}}\right\rangle\right): i \in I_{\psi, \varphi}\right\}>\max \bigcup_{J \in D_{\psi, \varphi}^{\prime}}\left\{g_{u}\left(\left|\varphi^{\max J}\right\rangle\right),\right. \\
& \left.g_{u}\left(\left|\varphi^{\min { }^{\psi, \varphi}}\right\rangle\right)\right\},
\end{aligned}
$$

then $|\chi\rangle$ can do partial entanglement recovery for the transformation of $|\psi\rangle$ to $|\varphi\rangle$.

Moreover, if $|\chi\rangle$ satisfies Eqs. (29)-(31), then there exists a positive number $\delta$ such that for any state $|\omega\rangle$ with a

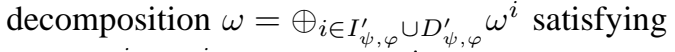

(i) $\omega^{i}=\chi^{i}$ for any $i \in I_{\psi, \varphi}^{\prime}$; and

(ii) $\left\|\omega^{i}-\chi^{i}\right\|<\delta$ and $\sum \omega^{i}=\sum \chi^{i}$ for any $i \in D_{\psi, \varphi}^{\prime}$, the transformation of $|\psi\rangle \otimes|\chi\rangle$ to $|\varphi\rangle \otimes|\omega\rangle$ can be realized with certainty by LOCC, i.e., $|\psi\rangle \otimes|\chi\rangle \rightarrow|\varphi\rangle \otimes|\omega\rangle$.

Proof. The proof is similar to Theorem 4.1, and we omit the details.

The key idea in the above theorem is to let all vectors between $\varphi^{k_{i}+1}$ and $\varphi^{k_{i+1}-1}$ correspond to a single $\chi^{D_{i}}$. This reduces the dimension of $\chi$ efficiently.

An interesting special case of Theorem 4.2 is when the majorization $\psi \prec \varphi$ splits into $m$ strict majorizations: $\psi^{i} \triangleleft \varphi^{i}$. We state this result in the following:

Corollary 4.3: Let $|\psi\rangle$ and $|\varphi\rangle$ be two states such that $|\psi\rangle$ is in $S(|\varphi\rangle)$. Suppose that $\psi$ and $\varphi$ have normal decompositions $\psi=\left(\psi^{1}, \ldots, \psi^{m}\right)$ and $\varphi=\left(\varphi^{1}, \ldots, \varphi^{m}\right)$, and let $I_{\psi, \varphi}=\emptyset$. If $|\chi\rangle$ is an auxiliary state such that

$$
l_{u}(|\chi\rangle)>\max \left\{g_{u}\left(\left|\varphi^{i}\right\rangle\right): 1 \leq i \leq m\right\},
$$

then $|\chi\rangle$ can do partial entanglement recovery for the transformation of $|\psi\rangle$ to $|\varphi\rangle$.

Proof. In fact, in this special case, $D_{\psi, \varphi}^{\prime}=\left\{D_{\psi, \varphi}\right\}=$ $\{\{1, \ldots, m\}\}, I_{\psi, \varphi}^{\prime}=\emptyset$. Thus, by Theorem 4.2 to do partial entanglement recovery, the only non-trivial condition that $|\chi\rangle$ should satisfy is Eq. 30, which is exactly the assumption of the present corollary.

It is easy to check that in the above corollary $|\chi\rangle$ can be chosen as a $2 \times 2$ state. However, by Theorem 4.1 we can only find a state $|\chi\rangle$ of dimension at least $2 m \times 2 m$.

By summarizing Theorems 3.2, 4.1, and 4.2, we have the following:

Theorem 4.3: Suppose that $|\psi\rangle$ and $|\varphi\rangle$ are two $n \times n$ states such that $\psi \prec \varphi$. We can always find an auxiliary state $|\chi\rangle$ to do partial entanglement recovery for the transformation of $|\psi\rangle$ to $|\varphi\rangle$, where the dimension of $\chi$ is between $2 \times 2$ and $n \times n$. Moreover, such a state $|\chi\rangle$ can only depend on the target state $|\varphi\rangle$ and the presence of equalities in the majorization $\psi \prec \varphi$.

Proof. The proof follows immediately from Theorems 3.2 4.1 and 4.2

The upper bound $n \times n$ cannot always be reduced to $(n-$ $1) \times(n-1)$. We have seen in Example 4.3 that when $n=4$, an auxiliary state $|\chi\rangle$ of dimension $4 \times 4$ is needed to do partial entanglement recovery for the transformation of $|\psi\rangle$ to $|\varphi\rangle$ such that $\Delta_{\psi, \varphi}=\{1,3\}$. 
We conclude this section by giving an example to illustrate the use of Theorem 4.2. This example is taken from [13].

Example 4.4: Let $|\psi\rangle$ and $|\varphi\rangle$ be two $n \times n$ states such that $|\psi\rangle$ is in $S(|\varphi\rangle)(n>6)$. Assume that $\Delta_{\psi, \varphi}=\{2,3,5\}$. The goal here is to find an auxiliary state $|\chi\rangle$ to do partial entanglement recovery for the transformation of $|\psi\rangle$ to $|\varphi\rangle$.

To be specific, let $\varphi=\left(\beta_{1}, \ldots, \beta_{n}\right)$. It is easy to check that $\varphi$ has a normal decomposition $\varphi=\left(\varphi^{1}, \varphi^{2}, \varphi^{3}, \varphi^{4}\right)$, where $\varphi^{1}=\left(\beta_{1}, \beta_{2}\right), \varphi^{2}=\left(\beta_{3}\right), \varphi^{3}=\left(\beta_{4}, \beta_{5}\right)$, and $\varphi^{4}=\left(\beta_{6}, \ldots, \beta_{n}\right)$. Also, $I_{\psi, \varphi}=\{2\}$ and $D_{\psi, \varphi}=\{1,3,4\}$. So $I_{\psi, \varphi}^{\prime}=\{\{2\}\}$ and $D_{\psi, \varphi}^{\prime}=\{\{1\},\{3,4\}\}$.

Take a $5 \times 5$ auxiliary state $|\chi\rangle$ with $\chi=$ $\left(\chi^{\{1\}}, \chi^{\{2\}}, \chi^{\{3,4\}}\right)$, where $\chi^{\{1\}}=\left(\gamma_{1}, \gamma_{2}\right), \chi^{\{2\}}=\left(\gamma_{3}\right)$, $\chi^{\{3,4\}}=\left(\gamma_{4}, \gamma_{5}\right)$, and $\gamma_{1}>\cdots>\gamma_{5}>0$. By Theorem 4.2. Eq. (29) yields

$$
\frac{\chi^{\{2\}}}{\chi^{\{1\}} \sqsupset \frac{\varphi^{2}}{\varphi^{1}}}
$$

and

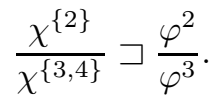

Eq. (30) yields

$$
\begin{aligned}
\min \left\{l_{u}\left(\left|\chi^{\{1\}}\right\rangle\right), l_{u}\left(\left|\chi^{\{3,4\}}\right\rangle\right)\right\}> & \max \left\{g_{u}\left(\left|\varphi^{1}\right\rangle\right),\right. \\
& \left.g_{u}\left(\left|\varphi^{3}\right\rangle\right), g_{u}\left(\left|\varphi^{4}\right\rangle\right)\right\} .
\end{aligned}
$$

Eq. (31) yields

$$
l_{u}\left(\left|\chi^{\{2\}}\right\rangle\right)>\max \left\{g_{u}\left(\left|\varphi^{1}\right\rangle\right), g_{u}\left(\left|\varphi^{3}\right\rangle\right), g_{u}\left(\left|\varphi^{4}\right\rangle\right)\right\},
$$

which is automatically satisfied since $l_{u}\left(\left|\chi^{\{2\}}\right\rangle\right)=1$ while the right hand side of Eq. (35) is strictly less than 1.

More explicitly, we have

$$
\frac{\gamma_{3}}{\gamma_{1}}>\frac{\beta_{3}}{\beta_{1}} \text { and } \frac{\gamma_{3}}{\gamma_{2}}<\frac{\beta_{3}}{\beta_{2}},
$$

and

$$
\frac{\gamma_{3}}{\gamma_{4}}>\frac{\beta_{3}}{\beta_{4}} \text { and } \frac{\gamma_{3}}{\gamma_{5}}<\frac{\beta_{3}}{\beta_{5}}
$$

and

$$
\min \left\{\frac{\gamma_{2}}{\gamma_{1}}, \frac{\gamma_{5}}{\gamma_{4}}\right)>\max \left\{\frac{\beta_{2}}{\beta_{1}}, \frac{\beta_{5}}{\beta_{4}}, \frac{\beta_{n}}{\beta_{6}}\right\} .
$$

With a routine calculation one can check that

$$
\begin{gathered}
\gamma_{1}=\mu \gamma_{3} \frac{\beta_{1}}{\beta_{3}}, \\
\gamma_{2}=(1+\eta) \gamma_{3} \frac{\beta_{2}}{\beta_{3}}, \\
\gamma_{4}=h \gamma_{3} \frac{\beta_{4}}{\beta_{3}}, \\
\gamma_{5}=(1+\lambda) \gamma_{3} \frac{\beta_{5}}{\beta_{3}}
\end{gathered}
$$

is a solution of the system of inequalities defined by Eqs. (36)-(38), where $\gamma_{3}>0$ is used to satisfy the normalization condition:

$$
\sum_{i=1}^{5} \gamma_{i}=1
$$

The parameters $\eta, \lambda, \mu$, and $h$ satisfy

$$
0<\eta<\frac{\beta_{1}-\beta_{2}}{\beta_{2}}, \quad 0<\lambda<\frac{\beta_{4}-\beta_{5}}{\beta_{5}} .
$$

and

$$
\begin{aligned}
& (1+\eta) \frac{\beta_{2}}{\beta_{1}}<\mu<\min \left\{(1+\eta) \frac{\beta_{2} \beta_{4}}{\beta_{1} \beta_{5}},(1+\eta) \frac{\beta_{2} \beta_{6}}{\beta_{1} \beta_{n}}, 1\right\}, \\
& (1+\lambda) \frac{\beta_{5}}{\beta_{4}}<h<\min \left\{(1+\lambda) \frac{\beta_{1} \beta_{5}}{\beta_{2} \beta_{4}},(1+\eta) \frac{\beta_{5} \beta_{6}}{\beta_{4} \beta_{n}}, 1\right\} .
\end{aligned}
$$

Notice that $\beta_{1}>\beta_{2}, \beta_{4}>\beta_{5}$, and $\beta_{6}>\beta_{n}$, and such a state $|\chi\rangle$ with $\chi=\left(\gamma_{1}, \ldots, \gamma_{5}\right)$ always exists. So we have actually constructed a class of states $|\chi\rangle$ with dimension $5 \times 5$ that can do partial entanglement recovery for the transformation of $|\psi\rangle$ to $|\varphi\rangle$.

\section{A POLYNOMIAL TIME ALGORITHM FOR PARTIAL ENTANGLEMENT RECOVERY}

In this section we study partial entanglement recovery from the algorithmic viewpoint. We present a polynomial algorithm of time complexity $O\left(n^{2} k^{4}\right)$ to decide whether $|\chi\rangle$ can be used to recover some entanglement lost in the transformation of $|\psi\rangle$ to $|\varphi\rangle$, where $n$ and $k$ are the dimensions of $\varphi$ and $\chi$, respectively.

The key part of Problem 1 is to solve the majorization relation $\psi \otimes \chi \prec \varphi \otimes \omega$. As argued before, the main difficulty here is how to deal with the order of the tensor product $\varphi \otimes \omega$ when $\omega$ varies. We will develop some techniques to overcome this difficulty. Notice that the map from $\omega$ to $\varphi \otimes \omega$ is an affine one. To make our discussions more general and more readable, in what follows we consider affine maps instead of tensor products.

To be concise, some concepts are introduced first.

Definition 5.1: Let $f$ and $g$ be real functions defined on $\mathcal{R}^{m}$, and let $\mathcal{S} \subset \mathcal{R}^{m}$. $f$ and $g$ are said to be comparable on $\mathcal{S}$ if

(i) $\forall x \in \mathcal{S}, f(x) \geq g(x)$; or

(ii) $\forall x \in \mathcal{S}, f(x) \leq g(x)$.

Let $F$ be a map from $\mathcal{R}^{m}$ to $\mathcal{R}^{n}$. We write $F(x)=$ $\left(f_{1}(x), \cdots, f_{n}(x)\right)$, where each $f_{i}$ is a real function defined on $\mathcal{R}^{m}$.

Definition 5.2: $F$ is said to have a fixed order on $\mathcal{S}$ if for any $1 \leq i<j \leq n, f_{i}$ and $f_{j}$ are comparable on $\mathcal{S}$.

Suppose $F$ has a fixed order on $\mathcal{S}$, and assume that whether $f_{i}$ and $f_{j}$ are comparable on $\mathcal{S}$ can be determined in $O(1)$ time. Then there exists a common algorithm which can sort the entries of $F(x)$ into non-increasing order for any $x \in \mathcal{S}$ in $O\left(n \log _{2} n\right)$ time. This fact is extremely useful in the following discussions.

If some entries of $F$ are not comparable on $\mathcal{S}$, then by the above definition $F$ does not have a fixed order. An important question naturally arises: how many different orders can $F$ have on $\mathcal{S}$ ?

Definition 5.3: $F$ is said to have at most $M$ different orders on $\mathcal{S}$ if there exists a decomposition of $\mathcal{S}$, say, $\mathcal{S}_{1}, \cdots, \mathcal{S}_{M}$, such that

(i) $\mathcal{S}=\mathcal{S}_{1} \cup \cdots \cup \mathcal{S}_{M}$; and 
(ii) $F$ has a fixed order on each $\mathcal{S}_{i}, i=1, \cdots, M$.

Now let $F$ be an affine map, $F(x)=A x+b$, where $A \in$ $\mathcal{R}^{n \times m}$ and $b \in \mathcal{R}^{n}$. At first glance, $F$ may have $n$ ! different orders on $\mathcal{R}^{m}$. However, this is not always true. A somewhat surprising fact is that the number of different orders of $F$ can be dramatically reduced to $O\left(n^{2 m}\right)$ when $m$ is a constant.

Lemma 5.1: $F$ has at most $O\left(n^{2 m}\right)$ different orders on $\mathcal{R}^{m}$.

Proof. For any $1 \leq i<j \leq n$, the difference of $f_{i}(x)$ and $f_{j}(x)$ crosses zero (from positive to negative, or negative to positive) if and only if $x$ crosses the hyperplane determined by the equation $f_{i}(x)-f_{j}(x)=0$, or more precisely,

$$
\Gamma_{i j}=\left\{\left(x_{1}, \cdots, x_{m}\right): \sum_{s=1}^{m}\left(a_{i s}-a_{j s}\right) x_{s}+\left(b_{i}-b_{j}\right)=0\right\} .
$$

It should be noted that there are two cases where $\Gamma_{i j}$ does not define a legal hyperplane. The first case is $\Gamma_{i j}=\emptyset$ and the second one is $\Gamma_{i j}=\mathcal{R}^{m}$. We will exclude these cases since in both of them $f_{i}$ and $f_{j}$ remain comparable whatever $x$ varies. Denote

$$
\Gamma=\left\{\Gamma_{i j}: \Gamma_{i j} \neq \emptyset \text { and } \Gamma_{i j} \neq \mathcal{R}^{m}, 1 \leq i<j \leq n\right\} .
$$

The number of hyperplanes in $\Gamma$ is less than or equal to $n(n-1) / 2$. These hyperplanes divide $\mathcal{R}^{m}$ into at most $O\left((n(n-1) / 2)^{m}\right)=O\left(n^{2 m}\right)$ different parts. $F$ has a fixed order on each part. With that we complete the proof.

It is obvious that the above lemma holds for any subset of $\mathcal{R}^{m}$.

Lemma 5.1 indicates that we can decompose $\mathcal{R}^{m}$ into $O\left(n^{2 m}\right)$ parts, $\mathcal{D}_{1}, \cdots, \mathcal{D}_{M}$, such that on each part, $F$ has a fixed order. In practice it is important to construct these parts explicitly. To see how this procedure can be done efficiently, let us first examine a special case where $m=1$.

Example 5.1: Let $F(x)=\left(a_{1} x-b_{1}, \cdots, a_{n} x-b_{n}\right)$, where $x \geq 0$. For simplicity assume $a_{i} \neq a_{j}, b_{i} \neq b_{j}, a_{i}, b_{i}>0$ for any $1 \leq i<j \leq n$.

By Lemma 5.1, $F$ has at most $O\left(n^{2}\right)$ different orders when $x$ varies as a non-negative number. In what follows we will show how to determine these orders explicitly.

Step 1. For each $1 \leq i<j \leq n$, solve equation $a_{i} x-b_{i}=$ $a_{j} x-b_{j}$. The solution is given by $\theta_{i j}=\left(b_{i}-b_{j}\right) /\left(a_{i}-a_{j}\right)$. Let $\Gamma=\left\{\theta_{i j}: 1 \leq i<j \leq n\right\} \cup\{0\}$. The number of elements of $\Gamma$ is denoted by $M$. It is easy to see that $M \leq n(n-1) / 2+1$.

Step 2. Sort the elements in $\Gamma$ into non-decreasing order, say $0=c_{0}<c_{1}<\cdots<c_{M-1}$.

Step 3. Construct a sequence of intervals: $\mathcal{D}_{1}=\left[c_{0}, c_{1}\right], \cdots$, $\mathcal{D}_{M}=\left[c_{M-1},+\infty\right)$.

It is clear that $F$ has a fixed order on each interval. The above procedure is completed in $O\left(n^{2}\right)+O\left(M \log _{2} M\right)+$ $O(M)=O\left(n^{2} \log _{2} n\right)$ time.

It is notable that the leftmost interval $\left[c_{0}, c_{1}\right]$ can be located in $O\left(n^{2}\right)$ time. This fact will be useful in the following discussions.

To deal with the general case, we need a lemma in computational geometry. Let $\mathcal{H}$ be a set of $n$ hyperplanes in $\mathcal{R}^{d}$ with $d>1$. Then $\mathcal{H}$ divides $\mathcal{R}^{d}$ into $O\left(n^{d}\right)$ parts with pairwise disjoint interiors. We call the set of these parts a $d$-arrangement of $\mathcal{H}$. A celebrated result in computational geometry shows that the $d$-arrangement of $\mathcal{H}$ can be enumerated efficiently [24].

Lemma 5.2: The $d$-arrangement of $n$ hyperplanes may be computed in time $O\left(n^{d}\right)$.

Employing Lemma 5.2, we can easily see that the above decomposition $\mathcal{D}_{1}, \cdots, \mathcal{D}_{M}$ can be computed in $O\left(n^{2 m}\right)$ time in the case that $m>1$.

With the aid of Lemma 5.1, we are able to solve a majorization inequality of the form $c \prec A x+b$ by using linear programming methods.

Lemma 5.3: The majorization inequality $c \prec F(x)$ can be solved in $O\left(n^{2 m+1} \log _{2} n\right)$ time, where $m$ is treated as a constant.

Proof By Lemma 5.1, $F$ has at most $M=O\left(n^{2 m}\right)$ different orders on $\mathcal{R}^{m}$. Let us decompose $\mathcal{R}^{m}$ into $M$ parts and enumerate them as $\mathcal{D}_{1}, \cdots, \mathcal{D}_{M}$. On each part $\mathcal{D}_{i}, F$ has a fixed order. This procedure needs time $O\left(n^{2 m}\right)$. In what follows we will show on each part, the majorization inequality $c \prec F(x)$ can be solved in $O\left(n \log _{2} n\right)$ time by using standard methods of linear programming. Hence we obtain an algorithm with time complexity $O\left(n^{2 m}\right)+O\left(n^{2 m} n \log _{2} n\right)=$ $O\left(n^{2 m+1} \log _{2} n\right)$ to solve the desired majorization inequality on $\mathcal{R}^{m}$.

Let us concentrate on a specific $\mathcal{D}_{i}$. An algorithm to solve the majorization inequality on $\mathcal{D}_{i}$ is as follows:

Step 1. Sort $c$ and $F(x)$ into non-increasing order, respectively. Assume $c^{\downarrow}=\left(c^{(1)}, \cdots, c^{(n)}\right)$ and $F^{\downarrow}(x)=$ $\left(a^{(1)}(x), \cdots, a^{(n)}(x)\right)$.

Step 2. Transform the majorization inequality $c \prec F(x)$ into the following linear system of inequalities:

$$
\sum_{s=1}^{l} c^{(s)} \leq \sum_{s=1}^{l} a^{(s)}(x), \quad 1 \leq l \leq n
$$

with equality holding when $l=n$.

Step 3. Solve the system of inequalities in Eq. (39) using standard techniques of linear programming.

Now let us calculate the time complexity of each step. It is obvious that $c$ can be sorted non-increasingly in $O\left(n \log _{2} n\right)$ time. Since $F$ has a fixed order on $\mathcal{D}_{i}, F(x)$ can also be sorted into non-increasing order in $O\left(n \log _{2} n\right)$ time. So Step 1 can be completed in $O\left(n \log _{2} n\right)$ time. To figure out the time complexity of Step 2, we need the following simple fact: the linear transform of $\left(y_{1}, \cdots, y_{n}\right)$ to $\left(y_{1}, y_{1}+y_{2}, \cdots, y_{1}+y_{2}+\right.$ $\left.\cdots+y_{n}\right)$ needs only $O(n)$ time. So Step 2 needs $O(n m)=$ $O(n)$ time. The time complexity of Step 3 needs a careful analysis. By applying the well-known Karmarkar's algorithm in the theory of linear programming [25] directly, Step 3 needs $O\left(n^{3.5}\right)$ time. However, in [26], it has been shown that linear programming can be solved in linear time $O(n)$ when the dimension of variable $x$ is fixed. Hence the total time to solve $c \prec F(x)$ on $D_{i}$ is

$$
O\left(n \log _{2} n\right)+O(n)+O(n)=O\left(n \log _{2} n\right) .
$$

With that we complete the proof of Lemma 5.3 
Now we are able to present our algorithms about partial entanglement recovery. The first algorithm to solve Problem 1 is a direct consequence of Lemma 5.3.

Theorem 5.1: Problem 1 is solvable in $O\left(n^{2 k-1} \log _{2} n\right)$ time, where $k$ is treated as a constant.

Proof. The key here is to solve the majorization inequality $\psi \otimes \chi \prec \varphi \otimes \omega$. Notice that when $\varphi$ is fixed, the map from $\omega$ to $\varphi \otimes$ is an affine one. So Lemma 5.3 works. A subtle point here is that $\omega$ is a $k$-dimensional probability vector and has only $k-1$ independent parameters. In addition, the relations $\omega \prec \chi$ and $\chi^{\downarrow} \neq \omega^{\downarrow}$ can easily be cast into linear constraints of $\omega$. The total number of these constraints is at most $O(k !)=O(1)$ when $k$ is a constant. Hence the time complexity is in fact $O\left((n k)^{2(k-1)+1} \log _{2} n\right)+O(1)=O\left(n^{2 k-1} \log _{2} n\right)$.

The main advantage of the above algorithm is that it can determine all the resulting states $|\omega\rangle$ in the process of partial entanglement recovery. However, this algorithm is efficient only when $k$ is treated as a constant. If $k$ varies freely, it will turn into exponential time complexity and cannot be efficient anymore. To further reduce the time complexity, some lemmas are necessary.

Let $\chi^{\downarrow}=\left(\gamma_{1}, \cdots, \gamma_{k}\right)$. For the sake of convenience, we assume all $k$ entries of $\chi$ are distinct. The general case can be considered similarly by using the compact form of $\chi$. For each $1 \leq i<j \leq k$ and $\epsilon>0$ we introduce the following vector:

$$
\chi(i, j, \epsilon)=\left(\gamma_{1}, \cdots, \gamma_{i}-\epsilon, \cdots, \gamma_{j}+\epsilon, \cdots, \gamma_{k}\right) .
$$

To keep the order of $\chi(i, j, \epsilon)$ fixed when $\epsilon$ varies, the constraints

$$
\gamma_{i}-\epsilon \geq \gamma_{i+1} \text { and } \gamma_{j-1} \geq \gamma_{j}+\epsilon
$$

should be satisfied. Let $\delta_{i j}$ be $\left(\gamma_{i}-\gamma_{i+1}\right) / 2$ if $j=i+1$, and be $\min \left\{\gamma_{i}-\gamma_{i+1}, \gamma_{j-1}-\gamma_{j}\right\}$ otherwise. Then $\epsilon \in\left[0, \delta_{i j}\right]$.

The following two lemmas exhibit some interesting properties of the solutions of Problem 1. Interestingly, the first lemma shows that we only need to consider the solution $|\omega\rangle$ with the Schmidt coefficient vector of a special form given in Eq. (40).

Lemma 5.4: Problem 1 has a solution if and only if there exist $1 \leq i<j \leq k$ and $\epsilon \in\left(0, \delta_{i j}\right]$ such that $\psi \otimes \chi \prec$ $\varphi \otimes \chi(i, j, \epsilon)$.

Proof. Sufficiency: Suppose such $i, j$, and $\epsilon$ do exist. It is easy to verify $\chi(i, j, \epsilon) \prec \chi$ and $\chi^{\downarrow} \neq \chi^{\downarrow}(i, j, \epsilon)$. These facts, together with the hypothesis $\psi \otimes \chi \prec \varphi \otimes \chi(i, j, \epsilon)$, indicate that $\chi(i, j, \epsilon)$ is a solution of Problem 1 .

Necessity: Assume Problem 1 has a solution $|\omega\rangle$. Then we have $\psi \otimes \chi \prec \varphi \otimes \omega, \omega \prec \chi$, and $\omega^{\downarrow} \neq \chi^{\downarrow}$. The existence of $i, j$, and $\epsilon$ such that $\psi \otimes \varphi \prec \varphi \otimes \chi(i, j, \epsilon)$ follows directly from the following two facts:

(a) If $\omega \prec \chi$ and $\chi^{\downarrow} \neq \omega^{\downarrow}$ then there exist $1 \leq i<j \leq k$ and $\epsilon \in\left(0, \delta_{i j}\right]$ such that $\omega \prec \chi(i, j, \epsilon) \prec \chi$. This is a direct consequence of Lemma 7.1 in Appendix B.

(b) Any state $\left|\chi^{\prime}\right\rangle$ such that $\omega \prec \chi^{\prime} \prec \chi$ and $\chi^{\downarrow} \neq \chi^{\prime \downarrow}$ is also a solution of Problem 1. This follows directly from our formulation of Problem 1.

Lemma 5.5: If $\left|\chi\left(i, j, \epsilon_{0}\right)\right\rangle$ is a solution of Problem 1, then for any $0<\epsilon<\epsilon_{0},|\chi(i, j, \epsilon)\rangle$ is also a solution.

Proof. Immediately from the formulation of Problem 1 and Eq. (40).

We are now in a position to state the main result of this section, the promised algorithm of time complexity $O\left(n^{2} k^{4}\right)$.

Theorem 5.2: Problem 1 is solvable in $O\left(n^{2} k^{4}\right)$ time.

Proof. By Lemma 5.4, we only need to consider the following problem: for each specific pair $(i, j)$ such that $1 \leq i<j \leq k$, decide whether there exists $\epsilon \in\left(0, \delta_{i j}\right]$ such that $\psi \otimes \chi \prec \varphi \otimes \chi(i, j, \epsilon)$. In what follows we show that this problem can be solved in $O\left(n^{2} k^{2}\right)$ time. Then by enumerating all possible pairs of $(i, j)$, we get an $O\left(k(k-1) / 2 n^{2} k^{2}\right)=$ $O\left(n^{2} k^{4}\right)$ time algorithm to solve Problem 1.

Let us begin with two specific indices $i$ and $j$. By Lemma 5.1. the number of the different orders of $\varphi \otimes \chi(i, j, \epsilon)$ is at most $O\left((n k)^{2}\right)$ when $\epsilon$ varies in $\left[0, \delta_{i j}\right]$. With Lemma 5.5 in mind, it is enough to consider one special order among them. More precisely, suppose the interval $\left[0, \delta_{i j}\right]$ is divided into $M$ parts (intervals), namely,

$$
\mathcal{D}_{1}=\left[c_{0}, c_{1}\right], \mathcal{D}_{2}=\left[c_{1}, c_{2}\right], \cdots, \mathcal{D}_{M}=\left[c_{M-1}, c_{M}\right],
$$

where $0=c_{0}<c_{1}<\cdots<c_{M}=\delta_{i j}$, and $M=O\left((n k)^{2}\right)$. On each interval $\varphi \otimes \chi(i, j, \epsilon)$ has a fixed order. By lemma 5.5 if $\left|\chi\left(i, j, \epsilon_{0}\right)\right\rangle$ is a solution of Problem 1 , then any $|\chi(i, j, \epsilon)\rangle$ such that $0<\epsilon \leq \min \left\{\epsilon_{0}, c_{1}\right\}$ is also a solution. So we need only to consider the leftmost interval $\mathcal{D}_{1}$. Our algorithm goes as follows:

Step 1: Find $c_{1}$;

Step 2: Sort $\psi \otimes \chi$ and $\varphi \otimes \chi(i, j, \epsilon)$ into non-increasing order, respectively, where $\epsilon \in\left[0, c_{1}\right]$;

Step 3: Solve the system of inequalities induced by the majorization relation $\psi \otimes \chi \prec \varphi \otimes \chi(i, j, \epsilon)$.

Step 4: Output: if a solution of $\epsilon>0$ is obtained in Step 3, then Problem 1 has a solution $|\chi(i, j, \epsilon)\rangle$; otherwise Problem 1 does not has a solution of the form $|\chi(i, j, \epsilon)\rangle$ for fixed $i$ and $j$, and $\epsilon \in\left(0, \delta_{i j}\right]$.

Step 1 requires that we search for the smallest positive elements among $M$ items, which requires $O(M)=O\left((n k)^{2}\right)$ time (see also Example 5.1). Step 2 needs $O\left(n k \log _{2} n k\right)$ time. Step 3 merely needs $O(n k)$ time since there is only a single parameter $\epsilon$. Step 4 only needs $O(1)$ time. In sum, only

$$
O\left((n k)^{2}\right)+O\left(n k \log _{2} n k\right)+O(n k)+O(1)=O\left((n k)^{2}\right)
$$

time is required.

In view of Theorem 5.2, we can say that Problem 1 is efficiently solvable. It also suggests that we can study the process of partial entanglement recovery using algorithmic methods.

To conclude our discussions about Problem 1, we would like to address an important issue for further study. In almost all the results we obtained so far, we are only concerned with the feasibility of partial entanglement recovery, while the 
efficiency of this process has not been touched yet. These results are of limited use in practice, when we hope to minimize entanglement lost in LOCC transformations. In other words, we require the resulting state $|\omega\rangle$ to be not only more entangled than $|\chi\rangle$, but also an "optimal" one that we can achieve in this process. Using entropy of entanglement as a measure, we suggest the following optimization problem. We also note that some aspects of the efficiency of partial entanglement recovery have been discussed in [14].

Open problem: Given a triple of states $(|\psi\rangle,|\varphi\rangle,|\chi\rangle)$ such that $\psi \prec \varphi$, let $\Omega=\{\omega: \psi \otimes \chi \prec \varphi \otimes \omega$ and $\omega \prec \chi\}$. Maximize $E(|\omega\rangle)$, subject to $\omega \in \Omega$.

In the above problem we remove the constraint $\chi^{\downarrow} \neq$ $\omega^{\downarrow}$. This makes $\Omega$ compact. Thus the continuous function $E(|\omega\rangle)$ can attain its maximum on $\Omega$. Suppose $\left|\omega_{0}\right\rangle$ is one of the states attaining the maximum. Noticing that the entropy of entanglement decreases under majorization, we have the following simple relation

$$
E(|\chi\rangle) \leq E\left(\left|\omega_{0}\right\rangle\right) \leq E(|\psi\rangle)-E(|\varphi\rangle)+E(|\chi\rangle),
$$

where the first inequality is from $\omega_{0} \prec \chi$, and the second inequality is from $\psi \otimes \chi \prec \varphi \otimes \omega_{0}$ and the additivity of entropy of entanglement. The first inequality is an equality if and only if $\omega^{\downarrow}=\chi^{\downarrow}$ for any $\omega \in \Omega$, i.e., $|\chi\rangle$ cannot do partial entanglement recovery for the transformation of $|\psi\rangle$ to $|\varphi\rangle$. The second inequality is an equality if and only if $(\psi \otimes$ $\chi)^{\downarrow}=\left(\varphi \otimes \omega_{0}\right)^{\downarrow}$. Theorem 5.2 in fact provides a polynomial time algorithm to determine whether the first inequality holds strictly. How to design efficient algorithms to find the optimal state $\left|\omega_{0}\right\rangle$ seems to be a challenging and worthwhile problem.

\section{SOME APPLICATIONS}

In this section, we establish some interesting connections of partial entanglement recovery to the generation of maximally entangled states, quantum catalysis, mutual catalysis, and multiple-copy entanglement transformation.

\section{A. How to obtain maximally entangled states by using partial entanglement recovery}

Maximally entangled states play a crucial role in many striking applications of quantum entanglement such as quantum superdense coding [2] and quantum teleportation [3]. It is very important to generate such states in practical information processing. Under the constraint of LOCC, a natural way to obtain a maximally entangled state is to concentrate a large number of partially entangled states [5]. However, such a concentrating protocol involves infinitely many copies of the source state while in practice only finitely many copies can be available. One can find various deterministic protocols based on Nielsen's theorem [6] and probabilistic protocols based on Vidal's theorem [17] (see also [16]). It has been shown that two $2 \times 2$ partially entangled states sometimes can be concentrated into an EPR pair deterministically [10]. An extensive generalization of such a deterministic concentration protocol was presented in [11], where the maximal number of Bell states that can be concentrated from a finite number of partially entangled states was derived. In what follows, we consider deterministic transformations only.

The following theorem shows that almost all deterministic entanglement transformations can concentrate a partially entangled pure state into a maximally entangled state with the same dimension providing that they are close enough to each other.

Theorem 6.1: Let $|\psi\rangle$ be a state in $S^{o}(|\varphi\rangle)$ and let $\left|\Phi^{+}\right\rangle=$ $\sum_{i=1}^{k} \frac{1}{\sqrt{k}}|i\rangle|i\rangle$ be a $k \times k$ maximally entangled state. Then there exists a positive number $\delta$ such that for any $k \times k$ state $|\chi\rangle$ satisfying $\||\chi\rangle-\left|\Phi^{+}\right\rangle \|<\delta$, the transformation of $|\psi\rangle \otimes|\chi\rangle$ to $|\varphi\rangle \otimes\left|\Phi^{+}\right\rangle$can be realized with certainty by LOCC, i.e., $|\psi\rangle \otimes|\chi\rangle \rightarrow|\varphi\rangle \otimes\left|\Phi^{+}\right\rangle$

Proof. This is a simple application of Lemma 2.3. Since $S^{o}(|\varphi\rangle)$ is not empty and $l_{u}\left(\left|\Phi^{+}\right\rangle\right)=1>g_{u}(|\varphi\rangle)$, it follows from Lemma 2.3 that

$$
\psi \otimes \Phi^{+} \triangleleft \varphi \otimes \Phi^{+} .
$$

An arbitrary but small enough perturbation on $\left|\Phi^{+}\right\rangle$in the left hand side of Eq. (41) can still keep the relation ' $\triangleleft$ '. Hence the existence of $\delta$ is proven.

The above theorem tells us that for any given $|\psi\rangle \in S^{o}(|\varphi\rangle)$ and $k>1$, we can find a partially entangled pure state $|\chi\rangle$ satisfying $|\psi\rangle \otimes|\chi\rangle \rightarrow|\varphi\rangle \otimes\left|\Phi^{+}\right\rangle$. It is obvious that $|\chi\rangle$ depends not only on $|\varphi\rangle$ and $k$, but also $|\psi\rangle$. At first glance, this seems to be contradicting our result about partial entanglement recovery, which states the auxiliary state $|\chi\rangle$ for partial entanglement recovery only depends on the target state $|\varphi\rangle$ and the presence of equalities in the majorization $\psi \prec \varphi$. The key point is when we consider whether $|\chi\rangle$ can be used to do partial entanglement recovery for a transformation with the target state $|\varphi\rangle$, the resulting state $|\omega\rangle$ is not specified; while the resulting state here is given and is maximally entangled. By Nielsen's theorem, $|\chi\rangle$ should be determined by the relation $\psi \otimes \chi \prec \varphi \otimes \Phi^{+}$, which obviously depends on the source state, the target state and $k$.

Theorem 6.1 confirms the existence of the partially entangled state $|\chi\rangle$. But it cannot yield a complete characterization of $|\chi\rangle$. To obtain such a characterization, we need to apply Nielsen's theorem and solve the corresponding majorization relation directly. To illustrate this procedure better, let us examine a simple case where $|\psi\rangle$ and $|\varphi\rangle$ are both $2 \times 2$ dimensional. In particular, the following example deals with the case of $k=2$.

Example 6.1: Let $|\psi\rangle,|\varphi\rangle$, and $\left|\Phi^{+}\right\rangle$be three $2 \times 2$ states with $\psi=(a, 1-a), \varphi=(b, 1-b)$, and $\Phi^{+}=\left(\frac{1}{2}, \frac{1}{2}\right)$, where $\frac{1}{2}<a<b \leq 1$. We are going to find a $2 \times 2$ partially entangled state $|\chi\rangle$ such that the transformation of $|\psi\rangle$ to $|\varphi\rangle$ can concentrate $|\chi\rangle$ into the maximally entangled state $\left|\Phi^{+}\right\rangle$.

Suppose that $|\chi\rangle$ is of the form $\chi=(p, 1-p)$, where $\frac{1}{2}<p<1$. By Nielsen's theorem, we only need $|\chi\rangle$ to satisfy

$$
\psi \otimes \chi \prec \varphi \otimes \Phi^{+} \text {. }
$$

Notice that $\varphi \otimes \Phi^{+}$has only two distinct components $\frac{1}{2} b$ and $\frac{1}{2}(1-b)$. By the definition of majorization, the above equation holds if and only if

$$
a p \leq \frac{1}{2} b
$$


and

$$
(1-a)(1-p) \geq \frac{1}{2}(1-b) .
$$

Hence

$$
\frac{1}{2}<p<\min \left\{\frac{b}{2 a}, \frac{1-2 a+b}{2(1-a)}\right\} .
$$

Note that $\frac{1}{2}<a<b<1$, so the above equation can be simplified into

$$
\frac{1}{2}<p \leq \frac{b}{2 a},
$$

which is exactly the result obtained in [10].

More generally, suppose that the $k \times k$ auxiliary state $|\chi\rangle$ is of the form $\chi=\left(\gamma_{1}, \ldots, \gamma_{k}\right)$. Then to obtain a $k \times k$ maximally entangled state $\left|\Phi^{+}\right\rangle$from the above transformation of $|\psi\rangle$ to $|\varphi\rangle$, it suffices to have $\psi \otimes \chi \prec \varphi \otimes \Phi^{+}$, which is equivalent to

$$
\gamma_{1} a \leq \frac{b}{k}
$$

and

$$
\gamma_{k}(1-a) \geq \frac{1-b}{k}
$$

Thus

$$
\frac{1-b}{k(1-a)} \leq \gamma_{k}<\gamma_{1} \leq \frac{b}{k a} .
$$

If $k=2$, we can show that Eq. (43) can be reduced to Eq. (42).

One can similarly consider the general case where both $k$ and $n$ are arbitrary positive integers.

\section{B. Partial entanglement recovery and quantum catalysis}

In the above discussions, we always assume that the source state $|\psi\rangle$ is comparable to the target state $|\varphi\rangle$, i.e., the transformation of $|\psi\rangle$ to $|\varphi\rangle$ can be realized with certainty under LOCC. How about the case where $|\psi\rangle$ and $|\varphi\rangle$ are not comparable? The general answer to this question remains unknown.

In [14] a special case where the transformation of $|\psi\rangle$ to $|\varphi\rangle$ has a catalyst state $|c\rangle$ such that $|\psi\rangle \otimes|c\rangle \rightarrow|\varphi\rangle \otimes|c\rangle$ [18], i.e., the transformation of $|\psi\rangle$ to $|\varphi\rangle$ can be realized under ELOCC, was examined carefully. It was shown that the problem of doing partial entanglement recovery for the transformation of $|\psi\rangle$ to $|\varphi\rangle$ with $\psi \nprec \varphi$ may be reduced to the problem of finding a catalyst state $|c\rangle$ and then seeking a suitable auxiliary state $|\chi\rangle$ to do partial entanglement recovery for the new transformation of $|\psi\rangle \otimes|c\rangle$ to $|\varphi\rangle \otimes|c\rangle$ such that $\psi \otimes c \prec \varphi \otimes c$. For this purpose, in [14] an algorithm of time complexity $O((n k) !)$ was proposed to find a $k \times k$ catalyst $|c\rangle$ for a transformation of $|\psi\rangle$ to $|\varphi\rangle$ in which the source state and the target state are both $n \times n$-dimensional.

However, the above algorithm is intractable since it is of exponential time complexity. In [22] a polynomial time algorithm of $n$ for fixed $k$ was given. With the aid of this efficient algorithm, one can quickly determine whether an $n \times n$ incomparable pair has a $k \times k$ catalyst. Then by the results obtained in the present paper, such as Theorems 3.2. 4.1, or 4.2. a state $|\chi\rangle$ that can do partial entanglement recovery for the transformation of $|\psi\rangle$ to $|\varphi\rangle$ can be explicitly constructed.
Therefore, if the transformation of $|\psi\rangle$ to $|\varphi\rangle$ can be realized with certainty under ELOCC, then we can find an auxiliary state $|\chi\rangle$ to do partial entanglement recovery for this transformation.

\section{Partial entanglement recovery and mutual catalysis}

In [23], an interesting phenomenon named mutual catalysis was demonstrated. If $|\psi\rangle \nrightarrow|\varphi\rangle$ and $|\alpha\rangle \nrightarrow|\beta\rangle$ but $|\psi\rangle \otimes$ $|\alpha\rangle \rightarrow|\varphi\rangle \otimes|\beta\rangle$, we say that $|\psi\rangle$ and $|\alpha\rangle$ can be mutually catalyzed by each other. The trivial case such that $|\psi\rangle \rightarrow|\beta\rangle$ and $|\alpha\rangle \rightarrow|\varphi\rangle$ is not necessary to consider. With the help of the results obtained in previous sections, one can easily construct many non-trivial instances with the mutual catalysis effect. First, let us reexamine an example from [23].

Example 6.2: Let $|\psi\rangle,|\varphi\rangle,|\alpha\rangle$, and $|\beta\rangle$ be four states with $\psi=(0.33,0.32,0.3,0.05), \varphi=(0.6,0.2,0.14,0.06), \alpha=$ $(0.6,0.3,0.1,0)$, and $\beta=(0.46,0.46,0.08,0)$. It is easy to see that both the transformations of $|\psi\rangle$ to $|\varphi\rangle$ and of $|\alpha\rangle$ to $|\beta\rangle$ cannot happen with certainty even under ELOCC. But we do have $|\psi\rangle \otimes|\alpha\rangle \rightarrow|\varphi\rangle \otimes|\beta\rangle$ in a non-trivial way. This is just the effect of mutual catalysis.

From another point of view, this example can be treated as a special instance of partial entanglement recovery. To see this, let us relabel the above four states as follows: $\chi=(0.6,0.3,0.1,0), \omega=(0.6,0.2,0.14,0.06)$, $\psi=(0.33,0.32,0.3,0.05)$, and $\varphi=(0.46,0.46,0.08,0)$. It is obvious that $\psi \triangleleft \varphi$. Noticing that $l_{u}(|\omega\rangle)>g_{u}(|\varphi\rangle)=0$, we have that $\psi \otimes \omega \triangleleft \varphi \otimes \omega$ by Lemma 2.3. A small perturbation on $\omega$ will generate $\chi=\omega+(0,0.1,-0.04,-0.06)$. Note that $E(|\chi\rangle)=1.2955<E(|\omega\rangle)=1.5472$. So the entropy of entanglement of $|\chi\rangle$ is enhanced.

The above example suggests a connection between partial entanglement recovery and mutual catalysis. More generally, any pairs $\{|\psi\rangle,|\chi\rangle\}$ and $\{|\varphi\rangle,|\omega\rangle\}$ such that $|\psi\rangle \otimes|\chi\rangle \rightarrow$ $|\varphi\rangle \otimes|\omega\rangle,|\chi\rangle \nrightarrow|\omega\rangle,|\chi\rangle \nrightarrow|\varphi\rangle$, and $|\psi\rangle \nrightarrow|\varphi\rangle \otimes|\omega\rangle$ can be treated as nontrivial instances of mutual catalysis. These pairs can be easily obtained with the aid of lemma 2.3. Furthermore, one can choose the state $|\omega\rangle$ satisfying $\omega \prec \chi$ but $\chi \nprec \omega$. We omit the construction details.

\section{Multiple-copy is essential for partial entanglement recov- ery}

Multiple-copy entanglement transformation is another interesting topic in quantum entanglement theory. Let us review this concept briefly. In [15], it was demonstrated that sometimes multiple copies of a source state may be transformed into the same number of copies of a target state although the transformation cannot happen for a single copy. That is, for some states $|\psi\rangle$ and $|\varphi\rangle$, although the transformation of $|\psi\rangle$ to $|\varphi\rangle$ cannot be realized with certainty by LOCC, there may exist $m>1$ such that the transformation of $|\psi\rangle^{\otimes m}$ to $|\varphi\rangle^{\otimes m}$ can be achieved deterministically. This kind of transformation that uses multiple copies of a source state and then transforms all of them together into the same number of copies of a target state is intuitively called 'multiple-copy entanglement 
transformation', or MLOCC for short. See [15], [19], [20], and [21] for more about MLOCC.

It may be of interest to study the relations between partial entanglement recovery and multiple-copy entanglement transformation. To our surprise, entanglement lost in a multiplecopy entanglement transformation can be recovered more easily than that in a single-copy transformation when the auxiliary state is specified. To demonstrate this point, we need the following theorem as a useful tool.

Theorem 6.2: Let $|\varphi\rangle$ and $|\chi\rangle$ be two partially entangled states. If $|\chi\rangle$ has at least two distinct nonzero Schmidt coefficients, then there exists a positive integer $k_{0}$ such that for any $k \geq k_{0}$ and $|\psi\rangle \in S^{o}(|\varphi\rangle)$, entanglement lost in the transformation of $|\psi\rangle^{\otimes k}$ to $|\varphi\rangle^{\otimes k}$ can be partially recovered by $|\chi\rangle$.

The most interesting part of the above theorem is that the choice of $k_{0}$ only depends on $|\chi\rangle$ and $|\varphi\rangle$.

Proof. First, applying Lemma 1 in [20] yields that $\psi \triangleleft \varphi$ implies $\psi^{\otimes k} \triangleleft \varphi^{\otimes k}$ for any $k \geq 1$. Second, notice that $g_{u}\left(|\varphi\rangle^{\otimes k}\right)=g_{u}^{k}(|\varphi\rangle)$ and $g_{u}(|\varphi\rangle)<1$. By the assumption on $|\chi\rangle$, we have $0<L_{u}(|\chi\rangle)<1$. Thus there exists $k_{0} \geq 1$ such that $L_{u}(|\chi\rangle)>g_{u}^{k}(|\varphi\rangle)$ for any $k \geq k_{0}$. Therefore, by Theorem 3.2. we deduce that $|\chi\rangle$ can be used to do partial entanglement recovery for the transformation of $|\psi\rangle^{\otimes k}$ to $|\varphi\rangle^{\otimes k}$ such that $k \geq k_{0}$ and $|\psi\rangle \in S^{o}(|\varphi\rangle)$.

Let us take now two states $|\varphi\rangle$ and $|\chi\rangle$ such that $0<$ $L_{u}(|\chi\rangle)<g_{u}(|\varphi\rangle)<1$. By Theorem 3.2, $|\chi\rangle$ cannot do partial entanglement recovery for any transformation with the target $|\varphi\rangle$ since $L_{u}(|\chi\rangle)<g_{u}(|\varphi\rangle)$. On the other hand, it is easy to see that $|\varphi\rangle$ and $|\chi\rangle$ satisfy the assumptions of Theorem 6.2 Hence, there exists $k_{0}$ such that for any $k \geq k_{0}$ and $|\psi\rangle \in S^{o}(|\varphi\rangle)$, entanglement lost in the $k$-copy transformation, i.e., the transformation of $|\psi\rangle^{\otimes k}$ to $|\varphi\rangle^{\otimes k}$, can be partially recovered by $|\chi\rangle$.

We give an intuitive explanation for the above theorem. If the auxiliary state $|\chi\rangle$ cannot do partial entanglement recovery for the transformations with the target state $|\varphi\rangle$, then the target state is too uniform, and it is too entangled relative to $|\chi\rangle$. So for any state $|\psi\rangle$ that can be transformed into $|\varphi\rangle$ under LOCC, the extra entanglement left (except the necessary part to finish the transformation of $|\psi\rangle$ to $|\varphi\rangle$ ) is not enough to be transferred into $|\chi\rangle$. But if multiple copies of the source state are provided, the extra entanglement will accumulate. Such extra entanglement can be transferred into the state $|\chi\rangle$ when it exceeds a threshold.

It is also interesting to investigate the partial entanglement recovery power when multiple copies of the auxiliary state $|\chi\rangle$ are available. We restrict ourselves to the special case that $|\chi\rangle$ is $2 \times 2$-dimensional. A surprising result appears as the following:

Theorem 6.3: Let $|\chi\rangle$ be a $2 \times 2$-dimensional partially entangled state and $|\varphi\rangle$ be any partially entangled state. If $L_{u}(|\chi\rangle)<g_{u}(|\varphi\rangle)$, then for any $k \geq 1,|\chi\rangle^{\otimes k}$ cannot do partial entanglement recovery for any transformation with target state $|\varphi\rangle$.

Intuitively, if the auxiliary state $|\chi\rangle$ is $2 \times 2$-dimensional, then more copies of $|\chi\rangle$ do not provide any extra power of partial entanglement recovery if $L_{u}(|\chi\rangle)<g_{u}(|\varphi\rangle)$. This is very reasonable since the key point of such a recovery is the difference between the entanglement resource of the source state and that of the target state, which keeps invariant during the process of increasing the number of copies of the auxiliary state.

Proof. By (3) of Lemma 2.2 we have that $L_{u}\left(|\chi\rangle^{\otimes k}\right)=L_{u}(|\chi\rangle)$ since $|\chi\rangle$ is a $2 \times 2$ state. So by the assumption $L_{u}(|\psi\rangle)<g_{u}(|\varphi\rangle)$, it follows from Theorem 3.2 that $|\chi\rangle^{\otimes k}$ cannot be used to do partial entanglement recovery for any transformation with the target state $|\varphi\rangle$.

In the case when $L_{u}(|\chi\rangle)=g_{u}(|\varphi\rangle)$, however, the partial entanglement recovery capability of $|\chi\rangle^{\otimes k}$ may be strictly more powerful than that of $|\chi\rangle$ for suitably large $k$. That is, $|\chi\rangle^{\otimes k}$ can do partial entanglement recovery for some transformation with the target state $|\varphi\rangle$ while $|\chi\rangle$ cannot. See the following example.

Example 6.3: Let $|\chi\rangle$ be a state with $\chi=(p, 1-p)$, where $\frac{1}{2}\langle p<1$. Then by Theorem 3.2 we know that $\mid \chi\rangle$ cannot do partial entanglement recovery for any transformation with target state $|\varphi\rangle$ such that $\varphi=(p, p, p, p, 1-p, 1-p) /(2+2 p)$.

However, by Theorem 3.2 again, $|\chi\rangle^{\otimes 2}$ with $\chi^{\otimes 2}=\left(p^{2}, p(1-p), p(1-p),(1-p)^{2}\right)$ can do partial entanglement recovery for any transformation of $|\psi\rangle$ to $|\varphi\rangle$ such that $|\psi\rangle$ is in $S^{o}(|\varphi\rangle)$.

A more general result in this special case is: if $|\varphi\rangle$ has only two distinct nonzero Schmidt coefficients, then for a sufficiently large $k,|\chi\rangle^{\otimes k}$ can always do partial entanglement recovery for any transformation of $|\psi\rangle$ to $|\varphi\rangle$ with $|\psi\rangle \in$ $S^{o}(|\varphi\rangle)$; otherwise such a recovery is impossible for arbitrarily large $k$.

\section{CONCLUSION}

To summarize, we obtain a complete characterization of an auxiliary bipartite entangled state $|\chi\rangle$ that can do partial entanglement recovery for the transformation of $|\psi\rangle$ to $|\varphi\rangle$ where $\psi$ is strictly majorized by $\varphi$. It is interesting that the choice of the auxiliary state can only depend on the target state $|\varphi\rangle$ and the presence of the equalities in the majorization relation $\psi \prec \varphi$. We further propose two sufficient conditions for $|\chi\rangle$ that can be used to do partial entanglement recovery for a class of transformations of $|\psi\rangle$ to $|\varphi\rangle$ with $\psi \prec \varphi$. We also study the feasibility of partial entanglement recovery from the algorithmic viewpoint. A polynomial algorithm of time complexity $O\left(n^{2} k^{4}\right)$ is presented for deciding the possibility of partial entanglement recovery. As applications, we establish some interesting connections of partial entanglement recovery to the generation of maximally entangled states, quantum catalysis, mutual catalysis, and multiple-copy entanglement transformation. We hope the results presented here may help us to manipulate quantum entanglement more economically.

\section{Appendix A: Proof of Lemma 2.3}

Take $|\psi\rangle \in S^{o}(|\varphi\rangle)$. Assume $\psi^{\downarrow}=\left(\alpha_{1}, \alpha_{2}, \ldots, \alpha_{n}\right), \varphi^{\downarrow}=$ $\left(\beta_{1}, \beta_{2}, \ldots, \beta_{n}\right)$, and $\chi^{\downarrow}=\left(\gamma_{1}, \gamma_{2}, \ldots, \gamma_{k}\right)$. If $k=1$ then the 
result follows trivially. In what follows we assume $k>1$.

First we prove that if $l_{u}(|\chi\rangle)>g_{u}(|\varphi\rangle)$ then $|\psi\rangle \otimes|\chi\rangle$ is in $S^{o}(|\varphi\rangle \otimes|\chi\rangle)$. In other words, we shall prove

$$
e_{l}(\psi \otimes \chi)<e_{l}(\varphi \otimes \chi)
$$

for any $1 \leq l<n k$.

We rewrite

$$
e_{l}(\psi \otimes \chi)=\sum_{i=1}^{k} e_{l_{i}}\left(\gamma_{i} \psi\right),
$$

where $0 \leq l_{i} \leq n$ and $\sum_{i=1}^{k} l_{i}=l$. Easily see that $\gamma_{i} \psi \triangleleft \gamma_{i} \varphi$ for all $1 \leq i \leq k$. So we merely need to consider the following two cases:

Case 1: There exists $1 \leq s \leq k$ such that $0<l_{s}<n$. In this case, $e_{l_{s}}\left(\gamma_{s} \psi\right)<e_{l_{s}}\left(\gamma_{s} \varphi\right)$ holds. Then Eq. (44) follows from

$$
\begin{aligned}
e_{l}(\psi \otimes \chi) & =\sum_{i=1}^{k} e_{l_{i}}\left(\gamma_{i} \psi\right) \\
& <\sum_{i=1}^{k} e_{l_{i}}\left(\gamma_{i} \varphi\right) \\
& \leq e_{l}(\varphi \otimes \chi),
\end{aligned}
$$

where the equality is by Eq. (45), and the second inequality is by the definition of $e_{l}(\varphi \otimes \chi)$.

Case 2: For any $1 \leq i \leq k, l_{i} \in\{0, n\}$. Let $h$ be the maximal index satisfying $l_{h}=n$. Then $1 \leq h<k$; otherwise $h=k$ implies $l=n k$, which contradicts the assumption $l<$ $n k$. Noticing $l_{u}(|\chi\rangle)>g_{u}(|\varphi\rangle)$, we have $\gamma_{h+1} / \gamma_{h}>\beta_{n} / \beta_{1}$, or

$$
\gamma_{h} \beta_{n}<\gamma_{h+1} \beta_{1} .
$$

By the definition of $e_{n h}(\psi \otimes \chi)$ and the assumption on $h$, we further have

$$
\begin{aligned}
e_{n h}(\psi \otimes \chi) & =\sum_{i=1}^{h} e_{n}\left(\gamma_{i} \psi\right) \\
& =\sum_{i=1}^{h-1} e_{n}\left(\gamma_{i} \varphi\right)+\sum_{i=1}^{n-1} \gamma_{h} \beta_{i}+\gamma_{h} \beta_{n},
\end{aligned}
$$

where the second equality is due to $e_{n}(\psi)=e_{n}(\varphi)$.

Substituting Eq. (46) into the above equation, we have

$$
\begin{aligned}
e_{n h}(\psi \otimes \chi) & <\sum_{i=1}^{h-1} e_{n}\left(\gamma_{i} \varphi\right)+\sum_{i=1}^{n-1} \gamma_{h} \beta_{i}+\gamma_{h+1} \beta_{1} \\
& \leq e_{n h}(\varphi \otimes \chi),
\end{aligned}
$$

where the second inequality is by the definition of $e_{n h}(\varphi \otimes \chi)$.

Therefore Eq. (44) holds for any $1 \leq l<n k$. By the definition, we have $|\psi\rangle \otimes|\chi\rangle$ is in $S^{o}(|\varphi\rangle \otimes|\chi\rangle)$. (Note that $e_{n k}(\psi \otimes \chi)=e_{n k}(\varphi \otimes \chi)$ holds for any $\chi$ whenever $\left.\psi \triangleleft \varphi\right)$.

Conversely, suppose $|\psi\rangle \otimes|\chi\rangle$ is in $S^{o}(|\varphi\rangle \otimes|\chi\rangle)$, while there exists some $1 \leq h<k$, such that

$$
\frac{\gamma_{h+1}}{\gamma_{h}} \leq \frac{\beta_{n}}{\beta_{1}},
$$

or equivalently,

$$
\gamma_{h} \beta_{n} \geq \gamma_{h+1} \beta_{1}
$$

Then we have

$$
\begin{aligned}
e_{n h}(\varphi \otimes \chi) & =\sum_{i=1}^{h} e_{n}\left(\gamma_{i} \varphi\right) \\
& =\sum_{i=1}^{h} e_{n}\left(\gamma_{i} \psi\right) \\
& \leq e_{n h}(\psi \otimes \chi),
\end{aligned}
$$

which contradicts the assumption that $e_{l}(\psi \otimes \chi)<e_{l}(\varphi \otimes \chi)$ for any $1 \leq l<n k$.

With that we complete the proof of Lemma 2.3

\section{APPEndix B: PRoof of Theorem 3.2}

To prove Theorem 3.2, we need the following three auxiliary facts about majorization.

Lemma 7.1: Let $y \in \mathcal{R}^{n}$ with compact form $y^{\downarrow}=$ $\left(y_{1}^{\prime \oplus k_{1}}, \ldots, y_{s}^{\prime \oplus k_{s}}\right)$, and let $x \in \mathcal{R}^{n}$ satisfying $x \prec y$ but $y \nprec x$. Then there exists $z \in \mathcal{R}^{n}$ such that $x \prec z \prec y$ and

$$
\begin{aligned}
z^{\downarrow}= & {\left[y_{1}^{\prime \oplus k_{1}}, \ldots, y_{i}^{\prime \oplus k_{i}-1}, y_{i}^{\prime}-\epsilon,\right.} \\
& \left.\ldots, y_{j}^{\prime}+\epsilon, y_{j}^{\prime \oplus k_{j}-1}, \ldots, y_{s}^{\prime \oplus k_{s}}\right],
\end{aligned}
$$

for some $1 \leq i<j \leq s$ and $\epsilon>0$.

Proof. This is a direct consequence of B.1. Lemma in [7] (page 21).

Lemma 7.2: If $x^{\prime} \prec y^{\prime}$ and $x^{\prime \prime} \prec y^{\prime \prime}$, then $\left(x^{\prime}, x^{\prime \prime}\right) \prec$ $\left(y^{\prime}, y^{\prime \prime}\right)$.

Proof. See part (i) of A.7. Lemma in [7] (page 121).

Lemma 7.3: Let $x^{\downarrow}=\left(x^{\prime \downarrow}, x^{\prime \prime \downarrow}\right)$ and $y^{\downarrow}=\left(y^{\prime} \downarrow, y^{\prime \prime} \downarrow\right)$. If $x \prec y$ and $x^{\prime} \prec y^{\prime}$ (or $x^{\prime \prime} \prec y^{\prime \prime}$ ), then $x^{\prime \prime} \prec y^{\prime \prime}$ (resp. $x^{\prime} \prec y^{\prime}$ ).

Proof. Suppose $x^{\prime}, y^{\prime} \in \mathcal{R}^{m}$ and $x^{\prime \prime}, y^{\prime \prime} \in \mathcal{R}^{n}$. By the assumption, we have

$$
e_{l}\left(x^{\prime \prime}\right)=e_{m+l}(x)-e_{m}\left(x^{\prime}\right)
$$

and

$$
e_{l}\left(y^{\prime \prime}\right)=e_{m+l}(y)-e_{m}\left(y^{\prime}\right)
$$

for any $1 \leq l \leq n$.

Noticing $x^{\prime} \prec y^{\prime}$ and $x \prec y$, we also have

$$
e_{m}\left(x^{\prime}\right)=e_{m}\left(y^{\prime}\right)
$$

and

$$
e_{l}(x) \leq e_{l}(y)
$$

for any $1 \leq l \leq m+n$ and the inequality is an equality if $l=m+n$. Thus Eqs. (47) - (50) give

$$
e_{l}\left(x^{\prime \prime}\right) \leq e_{l}\left(y^{\prime \prime}\right)
$$

for any $1 \leq l \leq n$, with the equality holding when $l=n$. That means $x^{\prime \prime} \prec y^{\prime \prime}$. If $x \prec y$ and $x^{\prime \prime} \prec y^{\prime \prime}$, we can prove $x^{\prime} \prec y^{\prime}$ similarly.

Now we proceed to the proof of Theorem 3.2 We first deal with the case where all the nonzero Schmidt coefficients of $|\chi\rangle$ are identical, i.e., $L_{u}(|\chi\rangle)=0$ or $L_{u}(|\chi\rangle)=1$.

Assume $\chi^{\downarrow}=\left(\left(\frac{1}{a}\right)^{\oplus a}, 0^{\oplus k-a}\right)$. If $a=k$, then $|\chi\rangle$ is a $k \times k$ maximally entangled state. There cannot be another $k \times k$ state $|\omega\rangle$ that is more entangled than $|\chi\rangle$. So partial entanglement recovery is not possible in this case.

Now suppose $1 \leq a<k$. If $|\chi\rangle$ can do partial entanglement recovery for some transformation of $|\psi\rangle$ to $|\varphi\rangle$ such that $|\psi\rangle$ is in $S(|\varphi\rangle)$, then the state $|\omega\rangle$ such that $\psi \otimes \chi \prec \varphi \otimes \omega$ and $\omega \prec \chi$, but $\chi^{\downarrow} \neq \omega^{\downarrow}$ should have at least $a+1$ nonzero Schmidt coefficients. By the property of majorization, the number of nonzero Schmidt coefficients of $\psi \otimes \chi$ is not less than that of $\varphi \otimes \omega$, i.e.,

$$
n^{\prime \prime} a \geq n^{\prime}(a+1)
$$


where $n^{\prime \prime}$ denotes the number of nonzero Schmidt coefficients of $|\psi\rangle$. Obviously, $n^{\prime \prime} \leq n$, thus we have

$$
n a \geq n^{\prime}(a+1) .
$$

Conversely, if Eq. (51) holds, we will show that $|\chi\rangle$ can be used to do partial entanglement recovery for any transformation of $|\psi\rangle$ to $|\varphi\rangle$ with $|\psi\rangle \in S^{o}(|\varphi\rangle)$. Let us take

$$
\chi(\epsilon)=\left(\left(\frac{1}{a}\right)^{\oplus a-1}, \frac{1}{a}-\epsilon, \epsilon, 0^{\oplus k-a-1}\right),
$$

where $\epsilon$ is a suitably small positive number. We have that

$$
\chi(\epsilon) \prec \chi \text { and } \chi^{\downarrow} \neq \chi^{\downarrow}(\epsilon)
$$

for any $0<\epsilon<\frac{1}{2 a}$. Noticing $|\psi\rangle \in S^{o}(|\varphi\rangle)$, by Lemma 2.3 we have

$$
\left(\psi \otimes\left(\frac{1}{a}\right)^{\oplus a}\right) \triangleleft\left(\varphi \otimes\left(\frac{1}{a}\right)^{\oplus a}\right) .
$$

Combining the above equation with Eq. (51) gives

$$
\left(\psi \otimes\left(\frac{1}{a}\right)^{\oplus a}\right) \triangleleft\left(\varphi \otimes\left(\left(\frac{1}{a}\right)^{\oplus a}, 0\right)\right)_{n a}^{1},
$$

where the notation $(x)_{j}^{i}$ denotes the segment $\left(x_{i}^{\downarrow}, \ldots, x_{j}^{\downarrow}\right)$ of $x^{\downarrow}$. Since a sufficiently small perturbation on the right-hand side of the above equation cannot change the relation $\triangleleft$, we have that

$$
\left(\psi \otimes\left(\frac{1}{a}\right)^{\oplus a}\right) \triangleleft\left(\varphi \otimes\left(\left(\frac{1}{a}\right)^{\oplus a-1}, \frac{1}{a}-\epsilon, \epsilon\right)\right)_{n a}^{1}
$$

for small enough positive number $\epsilon$.

Appending suitable number of zeros on the both sides of Eq. 52) gives

$$
\psi \otimes \chi \prec \varphi \otimes \chi(\epsilon),
$$

which proves that $|\chi\rangle$ can do partial entanglement recovery for the transformation of $|\psi\rangle$ to $|\varphi\rangle$.

Now we turn to the general case that $|\chi\rangle$ has at least two nonzero distinct Schmidt coefficients, i.e., $0<L_{u}(|\chi\rangle)<1$. We shall consider the following three cases: (i) $L_{u}(|\chi\rangle)>$ $g_{u}(|\varphi\rangle)$; (ii) $L_{u}(|\chi\rangle)<g_{u}(|\varphi\rangle)$; and (iii) $L_{u}(|\chi\rangle)=g_{u}(|\varphi\rangle)$.

First, we deal with case (i). We shall prove that if $L_{u}(|\chi\rangle)>$ $g_{u}(|\varphi\rangle)$, then $|\chi\rangle$ can do partial entanglement recovery for the transformation of $|\psi\rangle$ to $|\varphi\rangle$ such that $|\psi\rangle$ is in $S^{o}(|\varphi\rangle)$.

Suppose $\chi^{\downarrow}=\left(\gamma_{1}^{\oplus k_{1}}, \ldots, \gamma_{m}^{\oplus k_{m}}\right)$. Then there exists $1 \leq$ $i<m$ such that

$$
g_{u}(|\varphi\rangle)<L_{u}(|\chi\rangle)=\frac{\gamma_{i+1}}{\gamma_{i}}<1
$$

Let us denote $\left(\gamma_{1}^{\oplus k_{1}}, \ldots, \gamma_{i}^{\oplus k_{i}-1}\right), \quad\left(\gamma_{i}, \gamma_{i+1}\right)$, and $\left(\gamma_{i+1}^{\oplus k_{i+1}-1}, \ldots, \gamma_{m}^{\oplus k_{m}}\right)$ by $\gamma^{\prime}$, $\gamma^{\prime \prime}$, and $\gamma^{\prime \prime \prime}$, respectively. If $k_{i}=1$ or $k_{i+1}=1$, we simply omit the meaningless terms $\gamma^{\prime}$ or $\gamma^{\prime \prime \prime}$, respectively. For any $|\psi\rangle \in S^{o}(|\varphi\rangle)$, we have

$$
\begin{aligned}
& \psi \otimes \gamma^{\prime} \prec \varphi \otimes \gamma^{\prime}, \\
& \psi \otimes \gamma^{\prime \prime} \triangleleft \varphi \otimes \gamma^{\prime \prime},
\end{aligned}
$$

and

$$
\psi \otimes \gamma^{\prime \prime \prime} \prec \varphi \otimes \gamma^{\prime \prime \prime},
$$

where Eq. (55) comes from Eq. (53) and Lemma 2.3. So for a sufficiently small positive number $\epsilon$, we have

$$
\psi \otimes \gamma^{\prime \prime} \triangleleft \varphi \otimes \gamma^{\prime \prime}(\epsilon),
$$

where $\gamma^{\prime \prime}(\epsilon)=\left(\gamma_{i}-\epsilon, \gamma_{i+1}+\epsilon\right)$. By Eqs. (54), (56) and (57), applying Lemma 7.2 gives

$$
\psi \otimes \chi \prec \varphi \otimes \omega,
$$

where $\chi^{\downarrow}=\left(\gamma^{\prime}, \gamma^{\prime \prime}, \gamma^{\prime \prime}\right)$ and $\omega^{\downarrow}=\left(\gamma^{\prime}, \gamma^{\prime \prime}(\epsilon), \gamma^{\prime \prime \prime}\right)$. It is obvious that $\omega \prec \chi$ but $\chi^{\downarrow} \neq \omega^{\downarrow}$.

Second we deal with case (ii). Suppose that $L_{u}(|\chi\rangle)<$ $g_{u}(|\varphi\rangle)$. We shall prove that $|\chi\rangle$ cannot do partial entanglement recovery for any transformation $|\psi\rangle \rightarrow|\varphi\rangle$ such that $|\psi\rangle$ is in $S(|\varphi\rangle)$.

By contradiction, suppose that there exists a state $|\omega\rangle$ such that $\psi \otimes \chi \prec \varphi \otimes \omega, \omega \prec \chi$ and $\chi^{\downarrow} \neq \omega^{\downarrow}$. For any state $\left|\chi^{\prime}\right\rangle$ such that $\omega \prec \chi^{\prime} \prec \chi$ we have

$$
\psi \otimes \chi \prec \varphi \otimes \chi^{\prime},
$$

where we have used the assumption $\psi \otimes \chi \prec \varphi \otimes \omega$.

By Lemma 7.1, $\chi^{\prime}$ can be chosen as

$$
\chi^{\prime \downarrow}=\left(\gamma^{\prime}, \gamma^{\prime \prime}(\epsilon), \gamma^{\prime \prime \prime}\right),
$$

where $\gamma^{\prime}=\left(\gamma_{1}^{\oplus k_{1}}, \ldots, \gamma_{i}^{\oplus k_{i}-1}\right), \gamma^{\prime \prime}(\epsilon)=\left(\gamma_{i}-\epsilon, \ldots, \gamma_{j}+\epsilon\right)$, $\gamma^{\prime \prime \prime}=\left(\gamma_{j}^{\oplus k_{j}-1}, \ldots, \gamma_{m}^{\oplus k_{m}}\right), 1 \leq i<j \leq m$, and $\epsilon$ is an arbitrarily positive but small enough real number. In particular,

$$
\chi^{\downarrow}=\left(\gamma^{\prime}, \gamma^{\prime \prime}, \gamma^{\prime \prime \prime}\right),
$$

where $\gamma^{\prime \prime}=\gamma^{\prime \prime}(0)=\left(\gamma_{i}, \ldots, \gamma_{j}\right)$. However, we shall prove that such two indices $i$ and $j$ cannot exist, and thus complete the proof of this case.

For simplicity, let $n=\operatorname{dim}(\varphi)$. By the assumption $L_{u}(|\chi\rangle)<g_{u}(|\varphi\rangle)$, it follows that

$$
\gamma_{s} \beta_{n}>\gamma_{s+1} \beta_{1}, \text { for any } 1 \leq s \leq m-1,
$$

where $\beta_{1}$ and $\beta_{n}$ are the greatest and the least components of $\varphi$, respectively. Notice that $\psi \prec \varphi$. Applying part (6) of Lemma2.1 gives $g_{u}(|\psi\rangle) \geq g_{u}(|\varphi\rangle)$, thus $L_{u}(|\chi\rangle)<g_{u}(|\psi\rangle)$, or more explicitly,

$$
\gamma_{s} \alpha_{n}>\gamma_{s+1} \alpha_{1}, \text { for any } 1 \leq s \leq m-1,
$$

where $\alpha_{1}$ and $\alpha_{n}$ are the greatest and the least components of $\psi$, respectively.

Eqs. (60) and 62) imply

$$
(\psi \otimes \chi)^{\downarrow}=\left(\left(\psi \otimes \gamma^{\prime}\right)^{\downarrow},\left(\psi \otimes \gamma^{\prime \prime}\right)^{\downarrow},\left(\psi \otimes \gamma^{\prime \prime \prime}\right)^{\downarrow}\right) .
$$

Eqs. (59), 61), and $\epsilon>0$ imply

$$
\left(\varphi \otimes \chi^{\prime}\right)^{\downarrow}=\left(\left(\varphi \otimes \gamma^{\prime}\right)^{\downarrow},\left(\varphi \otimes \gamma^{\prime \prime}(\epsilon)\right)^{\downarrow},\left(\varphi \otimes \gamma^{\prime \prime \prime}\right)^{\downarrow}\right) .
$$

Applying Lemma 7.3 to Eqs. 63 and 64 yields

$$
\psi \otimes \gamma^{\prime \prime} \prec \varphi \otimes \gamma^{\prime \prime}(\epsilon),
$$

where we have used the assumption $\psi \prec \varphi$ and Eq. (58).

According to Eq. 61, we can take a sufficiently small positive number $\epsilon$ such that

$$
\left(\gamma_{i}-\epsilon\right) \beta_{n}>\left(\gamma_{i+1}+\epsilon\right) \beta_{1} .
$$


Then

$$
\begin{aligned}
e_{n}\left(\varphi \otimes \gamma^{\prime \prime}(\epsilon)\right) & =\left(\gamma_{i}-\epsilon\right) e_{n}(\varphi) \\
& <\gamma_{i} e_{n}(\psi) \\
& \leq e_{n}\left(\psi \otimes \gamma^{\prime \prime}\right),
\end{aligned}
$$

which contradicts Eq. (65).

Finally, we deal with case (iii), i.e., $L_{u}(|\chi\rangle)=g_{u}(|\varphi\rangle)$. This case is much more complicated than the previous two cases. It is in fact the most non-trivial part of Theorem 3.2 Since this case is of considerable interest, we will present a detailed proof for it. To keep the proof as readable as possible, the lengthy proof is divided into two easier lemmas. It is worth noting that both lemmas are interesting in their own right.

The first lemma shows that an auxiliary state can do partial entanglement recovery for a specific transformation if and only if some of its segments can do partial entanglement recovery for the same transformation.

Lemma 7.4: Let $|\psi\rangle$ and $|\varphi\rangle$ be two states such that $\psi \prec$ $\varphi$, and let $|\chi\rangle$ be a partially entangled state with compact form $\chi^{\downarrow}=\left(\gamma_{1}^{\oplus k_{1}}, \ldots, \gamma_{m}^{\oplus k_{m}}\right)$ for some $m>1$. If $L_{u}(|\chi\rangle)=$ $g_{u}(|\varphi\rangle)$, then the following two statements are equivalent:

(i) $|\chi\rangle$ can do partial entanglement recovery for the transformation of $|\psi\rangle$ to $|\varphi\rangle$;

(ii) There exists an index $i$ such that the unnormalized state $\left|\chi^{\prime}\right\rangle$ with $\chi^{\prime \downarrow}=\left(\gamma_{i}^{\oplus k_{i}}, \gamma_{i+1}^{\oplus k_{i+1}}\right)$ can do partial entanglement recovery for the transformation of $|\psi\rangle$ to $|\varphi\rangle$, where $1 \leq i<m$ and $\frac{\gamma_{i+1}}{\gamma_{i}}=g_{u}(|\varphi\rangle)$.

Proof. The essential part of the lemma is (i) $\Rightarrow$ (ii). Suppose that $|\chi\rangle$ can do partial entanglement recovery for the transformation of $|\psi\rangle$ to $|\varphi\rangle$. That is, there exists a state $|\omega\rangle$ satisfying $\psi \otimes \chi \prec \varphi \otimes \omega, \omega \prec \chi$, and $\chi^{\downarrow} \neq \omega^{\downarrow}$. Moreover, by Lemma 7.2, we can assume that $|\omega\rangle$ is of the following form:

$$
\omega^{\downarrow}=\left(\gamma^{\prime}, \gamma^{\prime \prime}(\epsilon), \gamma^{\prime \prime \prime}\right),
$$

where $\gamma^{\prime}=\left(\gamma_{1}^{\oplus k_{1}}, \ldots, \gamma_{i-1}^{\oplus k_{i-1}}\right), \gamma^{\prime \prime}(\epsilon)=\left(\gamma_{i}^{\oplus k_{i}-1}, \gamma_{i}-\right.$ $\left.\epsilon, \ldots, \gamma_{j}+\epsilon, \gamma_{j}^{\oplus k_{j}-1}\right), \gamma^{\prime \prime \prime}=\left(\gamma_{j+1}^{\oplus k_{j+1}}, \ldots, \gamma_{m}^{\oplus k_{m}}\right), 1 \leq i<$ $j \leq m$, and $\epsilon>0$. To make $\left(\gamma^{\prime \prime}(\epsilon)\right)^{\downarrow}=\gamma^{\prime \prime}(\epsilon)$ hold, we have assumed that $\epsilon$ satisfies $\gamma_{p}-\epsilon>\gamma_{p+1}+\epsilon$ for any $1 \leq p \leq m-1$. We also have

$$
\chi^{\downarrow}=\left(\gamma^{\prime}, \gamma^{\prime \prime}(0), \gamma^{\prime \prime \prime}\right),
$$

where $\gamma^{\prime \prime}(0)=\left(\gamma_{i}^{\oplus k_{i}}, \ldots, \gamma_{j}^{\oplus k_{j}}\right)$. have

By the assumptions $L_{u}(|\chi\rangle)=g_{u}(|\varphi\rangle)$ and $\psi \prec \varphi$, we

$$
(\psi \otimes \chi)^{\downarrow}=\left(\left(\psi \otimes \gamma^{\prime}\right)^{\downarrow},\left(\psi \otimes \gamma^{\prime \prime}(0)\right)^{\downarrow},\left(\psi \otimes \gamma^{\prime \prime \prime}\right)^{\downarrow}\right) .
$$

and

$$
(\varphi \otimes \omega)^{\downarrow}=\left(\left(\varphi \otimes \gamma^{\prime}\right)^{\downarrow},\left(\varphi \otimes \gamma^{\prime \prime}(\epsilon)\right)^{\downarrow},\left(\varphi \otimes \gamma^{\prime \prime \prime}\right)^{\downarrow}\right) .
$$

Applying Lemma 7.3 to Eqs. (66) and (67) yields

$$
\psi \otimes \gamma^{\prime \prime}(0) \prec \varphi \otimes \gamma^{\prime \prime}(\epsilon),
$$

where we have used the assumptions that $\psi \otimes \chi \prec \varphi \otimes \omega$ and $\psi \prec \varphi$.

Therefore, for the simplicity of notations and without any loss of generality, we can assume that $i=1$ and $j=m$. More directly, we can write $\gamma^{\prime \prime}(0)$ and $\gamma^{\prime \prime}(\epsilon)$ as $\chi$ and $\omega$, respectively. This, of course, will not cause any confusion.
We shall prove $m=2$ and $\frac{\gamma_{2}}{\gamma_{1}}=g_{u}(|\varphi\rangle)$ to complete the proof of the lemma.

For simplicity, we assume that $n=\operatorname{dim}(\psi)$ in the rest of proof.

First, we prove $1<m \leq 3$. By contradiction, suppose that $m>3$. Let us decompose

$$
\chi=\left(\chi^{\prime}(0), \chi^{\prime \prime}(0)\right)
$$

and

$$
\omega=\left(\chi^{\prime}(\epsilon), \chi^{\prime \prime}(\epsilon)\right)
$$

where

$$
\chi^{\prime}(\epsilon)=\left(\gamma_{1}^{\oplus k_{1}-1}, \gamma_{1}-\epsilon, \gamma_{2}^{\oplus k_{2}}\right)
$$

and

$$
\chi^{\prime \prime}(\epsilon)=\left(\gamma_{3}^{\oplus k_{3}}, \ldots, \gamma_{m}+\epsilon, \gamma_{m}^{\oplus k_{m}-1}\right) .
$$

Again, $L_{u}(|\chi\rangle)=g_{u}(|\varphi\rangle)$ and $\psi \prec \varphi$ give

$$
\gamma_{3} / \gamma_{2} \leq g_{u}(|\varphi\rangle) \text { and } \gamma_{3} / \gamma_{2} \leq g_{u}(|\psi\rangle) \text {. }
$$

That immediately yields

$$
(\psi \otimes \chi)^{\downarrow}=\left(\left(\psi \otimes \chi^{\prime}(0)\right)^{\downarrow},\left(\psi \otimes \chi^{\prime \prime}(0)\right)^{\downarrow}\right)
$$

and

$$
(\varphi \otimes \omega)^{\downarrow}=\left(\left(\varphi \otimes \chi^{\prime}(\epsilon)\right)^{\downarrow},\left(\varphi \otimes \chi^{\prime \prime}(\epsilon)\right)^{\downarrow}\right) .
$$

So,

$$
\begin{aligned}
e_{\left(k_{1}+k_{2}\right) n}(\psi \otimes \chi) & =e_{\left(k_{1}+k_{2}\right) n}\left(\psi \otimes \chi^{\prime}(0)\right) \\
& =k_{1} \gamma_{1}+k_{2} \gamma_{2}
\end{aligned}
$$

and

$$
\begin{aligned}
e_{\left(k_{1}+k_{2}\right) n}(\varphi \otimes \omega) & =e_{\left(k_{1}+k_{2}\right) n}\left(\varphi \otimes \chi^{\prime}(\epsilon)\right) \\
& =k_{1} \gamma_{1}+k_{2} \gamma_{2}-\epsilon,
\end{aligned}
$$

thus

$$
e_{\left(k_{1}+k_{2}\right) n}(\psi \otimes \chi)>e_{\left(k_{1}+k_{2}\right) n}(\varphi \otimes \omega)
$$

for any small $\epsilon>0$. This contradicts the assumption $\psi \otimes \chi \prec$ $\varphi \otimes \omega$. Hence $1<m \leq 3$

Second, we prove that for any $1 \leq i \leq m-1$, it holds that $\gamma_{i+1} / \gamma_{i}=\beta_{n} / \beta_{1}$, where $\beta_{1}$ and $\beta_{n}$ are the greatest and the least components of $\varphi$, respectively. By contradiction, we need to consider two cases: (1) $m=2$ and (2) $m=3$.

(1) $m=2$. Suppose that $\gamma_{2} / \gamma_{1}<\beta_{n} / \beta_{1}$. Let us choose a suitably small positive number $\epsilon$ such that

$$
\left(\gamma_{2}+\epsilon\right) \beta_{1}<\left(\gamma_{1}-\epsilon\right) \beta_{n} .
$$

A routine calculation shows that

$$
\begin{aligned}
e_{k_{1} n}(\psi \otimes \chi) & =e_{k_{1} n}\left(\psi \otimes \gamma_{1}^{\oplus k_{1}}\right) \\
& =k_{1} \gamma_{1}
\end{aligned}
$$

and

$$
\begin{aligned}
e_{k_{1} n}(\varphi \otimes \omega) & =e_{\left(k_{1}-1\right) n}\left(\varphi \otimes \gamma_{1}^{\oplus k_{1}-1}\right)+e_{n}\left(\left(\gamma_{1}-\epsilon\right) \varphi\right) \\
& =k_{1} \gamma_{1}-\epsilon,
\end{aligned}
$$

which yields

$$
e_{k_{1} n}(\psi \otimes \chi)>e_{k_{1} n}(\varphi \otimes \omega)
$$

for any small $\epsilon>0$. That again contradicts $\psi \otimes \chi \prec \varphi \otimes \omega$. 
(2) $m=3$. Suppose that $\gamma_{2} / \gamma_{1}<\beta_{n} / \beta_{1}$ or $\gamma_{3} / \gamma_{2}<$ $\beta_{n} / \beta_{1}$. We only consider the case where $\gamma_{3} / \gamma_{2}<\beta_{n} / \beta_{1}$, and the left case is similar to case (i). Choose a suitably small positive number $\epsilon$ such that

$$
\left(\gamma_{3}+\epsilon\right) \beta_{1}<\gamma_{2} \beta_{n}
$$

Then a simple analysis shows

$$
\begin{aligned}
e_{\left(k_{1}+k_{2}\right) n}(\psi \otimes \chi) & =e_{k_{1} n}\left(\psi \otimes \gamma_{1}^{\oplus k_{1}}\right)+e_{k_{2} n}\left(\psi \otimes \gamma_{2}^{\oplus k_{2}}\right) \\
& =k_{1} \gamma_{1}+k_{2} \gamma_{2}
\end{aligned}
$$

and

$$
\begin{aligned}
e_{\left(k_{1}+k_{2}\right) n}(\varphi \otimes \omega) & =e_{\left(k_{1}-1\right) n}\left(\varphi \otimes \gamma_{1}^{\oplus k_{1}-1}\right) \\
& +e_{n}\left(\left(\gamma_{1}-\epsilon\right) \varphi\right)+e_{k_{2} n}\left(\varphi \otimes \gamma_{2}^{\oplus k_{2}}\right) \\
& =k_{1} \gamma_{1}+k_{2} \gamma_{2}-\epsilon,
\end{aligned}
$$

which yields

$$
e_{\left(k_{1}+k_{2}\right) n}(\psi \otimes \chi)>e_{\left(k_{1}+k_{2}\right) n}(\varphi \otimes \omega) .
$$

That is a contradiction with $\psi \otimes \chi \prec \varphi \otimes \omega$.

Third, we prove that $m=2$. By contradiction, we shall show that if $m=3$ then

$$
\psi \otimes \chi \prec \varphi \otimes \omega
$$

cannot hold for any small enough positive number $\epsilon$, where $\chi^{\downarrow}=\left(\gamma_{1}^{\oplus k_{1}}, \gamma_{2}^{\oplus k_{2}}, \gamma_{3}^{\oplus k_{3}}\right), \omega^{\downarrow}=\left(\gamma_{1}^{\oplus k_{1}-1}, \gamma_{1}-\epsilon, \gamma_{2}^{\oplus k_{2}}, \gamma_{3}+\right.$ $\left.\epsilon, \gamma_{3}^{\oplus k_{3}-1}\right), \gamma_{2} / \gamma_{1}=\gamma_{3} / \gamma_{2}=g_{u}(|\varphi\rangle)$.

To be specific, let $\varphi^{\downarrow}=\left(\beta_{1}^{\oplus m_{1}}, \ldots, \beta_{h}^{\oplus m_{h}}\right)$ for some $h \geq 2$. Obviously, $n=\operatorname{dim}(\psi)=\sum_{t=1}^{h} m_{t}$. Choose $\epsilon$ such that

$$
\left(\gamma_{1}-\epsilon\right) \beta_{i}>\gamma_{1} \beta_{i+1}
$$

and

$$
\left(\gamma_{3}+\epsilon\right) \beta_{i+1}<\gamma_{3} \beta_{i}
$$

for any $1 \leq i \leq h-1$.

In addition to the above constraints, we also need $\epsilon$ satisfying

$$
\left(\gamma_{1}-\epsilon\right) \gamma_{h}>\left(\gamma_{3}+\epsilon\right) \beta_{1}
$$

A direct calculation gives

$$
(\psi \otimes \chi)^{\downarrow}=\left(\varphi^{\prime}(0), \varphi^{\prime \prime}(0)\right)
$$

and

$$
(\varphi \otimes \omega)^{\downarrow}=\left(\varphi^{\prime}(\epsilon), \varphi^{\prime \prime}(\epsilon)\right),
$$

where

$$
\begin{aligned}
\varphi^{\prime}(\epsilon)= & {\left[\gamma_{1} \beta_{1}^{\oplus\left(k_{1}-1\right) m_{1}},\left(\gamma_{1}-\epsilon\right) \beta_{1}^{\oplus m_{1}},\right.} \\
& \left.\ldots, \gamma_{1} \beta_{h-1}^{\oplus\left(k_{1}-1\right) m_{h-1}},\left(\gamma_{1}-\epsilon\right) \beta_{h-1}^{\oplus m_{h-1}}\right],
\end{aligned}
$$

and

$$
\begin{aligned}
\varphi^{\prime \prime}(\epsilon)= & {\left[\gamma_{1} \beta_{h}^{\oplus\left(k_{1}-1\right) m_{h}+k_{2} m_{1}},\left(\gamma_{1}-\epsilon\right) \beta_{h}^{\oplus m_{h}},\right.} \\
& \left.\ldots,\left(\gamma_{3}+\epsilon\right) \beta_{h}^{\oplus m_{h}}, \gamma_{3} \beta_{h}^{\oplus\left(k_{3}-1\right) m_{h}}\right],
\end{aligned}
$$

where we have used $\gamma_{1} \beta_{h}=\gamma_{2} \beta_{1}$.

It can be readily verified that

$$
\begin{aligned}
e_{k_{1} n}(\psi \otimes \chi) & =e_{k_{1}\left(n-m_{h}\right)}\left(\varphi^{\prime}(0)\right)+e_{k_{1} m_{h}}\left(\varphi^{\prime \prime}(0)\right) \\
& =k_{1} \gamma_{1}
\end{aligned}
$$

and

$$
\begin{aligned}
e_{k_{1} n}(\varphi \otimes \omega) & =e_{k_{1}\left(n-m_{h}\right)}\left(\varphi^{\prime}(\epsilon)\right)+e_{k_{1} m_{h}}\left(\varphi^{\prime \prime}(\epsilon)\right) \\
& =\left(k_{1} \gamma_{1}-\epsilon\right)\left(1-m_{h} \beta_{h}\right)+e_{k_{1} m_{h}}\left(\varphi^{\prime \prime}(\epsilon)\right) .
\end{aligned}
$$

To calculate $e_{k_{1} m_{h}}\left(\varphi^{\prime \prime}(\epsilon)\right)$, we need to consider the following two cases:

(a) $k_{2} m_{1} \geq m_{h}$. Then

$$
\begin{aligned}
e_{k_{1} m_{h}}\left(\varphi^{\prime \prime}(\epsilon)\right) & =e_{k_{1} m_{h}}\left(\gamma_{1} \beta_{h}^{\oplus k_{1} m_{h}}\right) \\
& =k_{1} m_{h} \gamma_{1} \beta_{h},
\end{aligned}
$$

thus

$$
\begin{aligned}
e_{k_{1} n}(\varphi \otimes \omega) & =k_{1} \gamma_{1}-\epsilon\left(1-m_{h} \beta_{h}\right) \\
& <e_{k_{1} n}(\psi \otimes \chi)
\end{aligned}
$$

providing $\epsilon>0$.

(b) $k_{2} m_{1}<m_{h}$. Then

$$
\begin{aligned}
e_{k_{1} m_{h}}\left(\varphi^{\prime \prime}(\epsilon)\right) & =e_{l_{1}}\left(\gamma_{1} \beta_{h}^{\oplus l_{1}}\right)+e_{l_{2}}\left(\left(\gamma_{1}-\epsilon\right) \beta_{h}^{\oplus l_{2}}\right) \\
& =k_{1} m_{h} \gamma_{1} \beta_{h}-\epsilon\left(m_{h}-k_{2} m_{1}\right) \beta_{h},
\end{aligned}
$$

where $l_{1}=\left(k_{1}-1\right) m_{h}+k_{2} m_{1}$ and $l_{2}=m_{h}-k_{2} m_{1}$. Thus

$$
\begin{aligned}
e_{k_{1} n}(\varphi \otimes \omega) & =k_{1} \gamma_{1}-\epsilon\left(1-k_{2} m_{1} \beta_{h}\right) \\
& <k_{1} \gamma_{1}-\epsilon\left(1-m_{h} \beta_{h}\right) \\
& <e_{k_{1} n}(\psi \otimes \chi)
\end{aligned}
$$

providing $\epsilon>0$. In the above two cases we have used $\gamma_{1} \beta_{h}=$ $\gamma_{2} \beta_{1}$ to simplify the calculations.

Both the above two cases contradict $\psi \otimes \chi \prec \varphi \otimes \omega$. Thus $m=3$ is impossible.

With that we complete the proof of Lemma 7.4

By Lemma 7.4, under the condition $L_{u}(|\chi\rangle)=g_{u}(|\varphi\rangle)$, we only need to consider a special form of $|\chi\rangle$. More precisely, $\chi$ has only two distinct components. The following lemma will just handle such a special form of $|\chi\rangle$.

Lemma 7.5: Let $|\chi\rangle$ be a partially entangled state with compact form $\chi^{\downarrow}=\left(\beta_{1}^{\oplus k_{1}}, \beta_{2}^{\oplus k_{2}}\right)$ for some $\beta_{1}>\beta_{2}>0$, and let $|\varphi\rangle$ be another state satisfying $g_{u}(|\varphi\rangle)=L_{u}(|\chi\rangle)$. Then $|\chi\rangle$ can do partial entanglement recovery for the transformation of $|\psi\rangle$ to $|\varphi\rangle$ such that $|\psi\rangle$ is in $S^{o}(|\varphi\rangle)$ if and only if

$$
\varphi^{\downarrow}=\left(\frac{\chi^{\prime \oplus m}}{C}\right)^{\downarrow},
$$

where $\chi^{\prime}$ is a segment of $\chi^{\downarrow}$ with two distinct components, $C$ is a normalization factor, and $m \geq 1$.

Moreover, if $|\chi\rangle$ and $|\varphi\rangle$ don't satisfy Eq. (69), then $|\chi\rangle$ cannot do partial entanglement recovery for any transformation of $|\psi\rangle$ to $|\varphi\rangle$ such that $|\psi\rangle$ is in $S(|\varphi\rangle)$.

Proof. We first prove that if $|\chi\rangle$ can do partial entanglement recovery for some transformation of $|\psi\rangle$ to $|\varphi\rangle$ with $\psi \prec \varphi$, i.e., there exists a state $|\omega\rangle$ satisfying $\psi \otimes \chi \prec \varphi \otimes \omega, \omega \prec \chi$, and $\chi^{\downarrow} \neq \omega^{\downarrow}$, then $|\chi\rangle$ and $|\varphi\rangle$ should satisfy Eq. (69).

Suppose that $|\varphi\rangle$ and $|\omega\rangle$ have compact forms

$$
\varphi^{\downarrow}=\left(\beta_{1}^{\oplus m_{1}}, \ldots, \beta_{h}^{\oplus m_{h}}\right)
$$

and

$$
\omega^{\downarrow}=\left(\gamma_{1}^{\oplus k_{1}-1}, \gamma_{1}-\epsilon, \gamma_{2}+\epsilon, \gamma_{2}^{\oplus k_{2}-1}\right) .
$$

We shall prove that if $\psi \otimes \chi \prec \varphi \otimes \omega$ and $\omega \prec \chi$ for any sufficiently small positive number $\epsilon$, then

$$
h=2 \text { and } \frac{1}{k_{2}} \leq \frac{m_{1}}{m_{2}} \leq k_{1} .
$$


Or more compactly, $\varphi$ has the form as in Eq. (69).

The condition $L_{u}(|\chi\rangle)=g_{u}(|\varphi\rangle)$ is equivalent to

$$
\gamma_{2} \beta_{1}=\gamma_{1} \beta_{h}
$$

For any $1 \leq i \leq h-1$, choose $\epsilon$ such that

$$
\left(\gamma_{1}-\epsilon\right) \beta_{i}>\gamma_{1} \beta_{i+1}
$$

and

$$
\left(\gamma_{2}+\epsilon\right) \beta_{i+1}<\gamma_{2} \beta_{i}
$$

In addition to the above conditions, we also choose $\epsilon$ satisfying

$$
\left(\gamma_{1}-\epsilon\right) \beta_{h-1}>\left(\gamma_{2}+\epsilon\right) \beta_{1}
$$

and

$$
\left(\gamma_{1}-\epsilon\right) \beta_{h}>\left(\gamma_{2}+\epsilon\right) \beta_{2}
$$

By the condition $L_{u}(|\chi\rangle)=g_{u}(|\varphi\rangle)$ and $\psi \prec \varphi$, it is easy to verify that

$$
(\psi \otimes \chi)^{\downarrow}=\left(\left(\psi \otimes \gamma_{1}^{\oplus k_{1}}\right)^{\downarrow},\left(\psi \otimes \gamma_{2}^{\oplus k_{2}}\right)^{\downarrow}\right) .
$$

Take

$$
n=\operatorname{dim}(\varphi)=\sum_{i=1}^{h} m_{i}
$$

Then by Eq. (76),

$$
e_{k_{1} n}(\psi \otimes \chi)=k_{1} \gamma_{1}
$$

By Eqs. (71)-(75), a careful analysis gives

$$
(\varphi \otimes \omega)^{\downarrow}=\left(\varphi^{\prime}, \varphi^{\prime \prime}\right),
$$

where

$$
\begin{aligned}
\varphi^{\prime}= & {\left[\gamma_{1} \beta_{1}^{\oplus m_{1}\left(k_{1}-1\right)},\left(\gamma_{1}-\epsilon\right) \beta_{1}^{\oplus m_{1}},\right.} \\
& \left.\ldots, \gamma_{1} \beta_{h-1}^{\oplus m_{h-1}\left(k_{1}-1\right)},\left(\gamma_{1}-\epsilon\right) \beta_{h-1}^{\oplus m_{h-1}}\right]
\end{aligned}
$$

and

$$
\begin{aligned}
& \varphi^{\prime \prime}=\left[\left(\gamma_{2}+\epsilon\right) \beta_{1}^{\oplus m_{1}}, \gamma_{1} \beta_{h}^{\oplus m_{h}\left(k_{1}-1\right)+m_{1}\left(k_{2}-1\right)},\right. \\
& \left.\left(\gamma_{1}-\epsilon\right) \beta_{h}^{\oplus m_{h}}, \ldots, \gamma_{2} \beta_{h}^{\oplus m_{h}\left(k_{2}-1\right)}\right] \text {. }
\end{aligned}
$$

So

$$
\begin{aligned}
e_{k_{1} n}(\varphi \otimes \omega) & =e_{k_{1}\left(n-m_{h}\right)}\left(\varphi^{\prime}\right)+e_{k_{1} m_{h}}\left(\varphi^{\prime \prime}\right) \\
& =\left(k_{1} \gamma_{1}-\epsilon\right)\left(1-m_{h} \beta_{h}\right)+e_{k_{1} m_{h}}\left(\varphi^{\prime \prime}\right) .
\end{aligned}
$$

We need to consider the following four cases according to the values of $e_{k_{1} m_{h}}\left(\varphi^{\prime \prime}\right)$ :

Case (a): $m_{1}>k_{1} m_{h}$. Then it is obvious that

$$
\begin{aligned}
e_{k_{1} m_{h}}\left(\varphi^{\prime \prime}\right) & =e_{k_{1} m_{h}}\left(\left(\gamma_{2}+\epsilon\right) \beta_{1}^{\oplus k_{1} m_{h}}\right) \\
& =k_{1} m_{h}\left(\gamma_{2}+\epsilon\right) \beta_{1} .
\end{aligned}
$$

Combining Eqs. (79) with (80), we have

$$
e_{k_{1} n}(\varphi \otimes \omega)=k_{1} \gamma_{1}+\epsilon\left(k_{1} m_{h} \beta_{1}+m_{h} \beta_{h}-1\right),
$$

where we have used the relation $\gamma_{1} \beta_{h}=\gamma_{2} \beta_{1}$ to simplify the calculations.

Since $\psi \otimes \chi \prec \varphi \otimes \omega$, it follows that $e_{k_{1} n}(\varphi \otimes \omega) \geq e_{k_{1} n}(\psi \otimes$ $\chi)$, i.e.,

$$
k_{1} \gamma_{1}+\epsilon\left(k_{1} m_{h} \beta_{1}+m_{h} \beta_{h}-1\right) \geq k_{1} \gamma_{1} .
$$

Or equivalently,

$$
k_{1} m_{h} \beta_{1}+m_{h} \beta_{h} \geq 1 .
$$

However, by $m_{1}>k_{1} m_{h}$ and $\sum_{i=1}^{h} m_{i} \beta_{i}=1$, it follows that

$$
k_{1} m_{h} \beta_{1}+m_{h} \beta_{h}<m_{1} \beta_{1}+m_{h} \beta_{h} \leq 1,
$$

which contradicts Eq. (81).

Case (b): $m_{h} \leq m_{1} \leq k_{1} m_{h}$. It is easy to calculate that

$$
\begin{aligned}
e_{k_{1} m_{h}}\left(\varphi^{\prime \prime}\right)= & e_{m_{1}}\left(\left(\gamma_{2}+\epsilon\right) \beta_{1}^{\oplus m_{1}}\right) \\
& +e_{k_{1} m_{h}-m_{1}}\left(\gamma_{1} \beta_{h}^{\oplus k_{1} m_{h}-m_{1}}\right) \\
= & \epsilon m_{1} \beta_{1}+k_{1} m_{h} \gamma_{1} \beta_{h} .
\end{aligned}
$$

By Eqs. (79) and (82), it follows that

$$
e_{k_{1} n}(\varphi \otimes \omega)=k_{1} \gamma_{1}+\epsilon\left(m_{1} \beta_{1}+m_{h} \beta_{h}-1\right) .
$$

Since $\psi \otimes \chi \prec \varphi \otimes \omega$, it follows that $e_{k_{1} n}(\varphi \otimes \omega) \geq e_{k_{1} n}(\psi \otimes$ $\chi)$, i.e.

$$
k_{1} \gamma_{1}+\epsilon\left(m_{1} \beta_{1}+m_{h} \beta_{h}-1\right) \geq k_{1} \gamma_{1} .
$$

Or equivalently,

$$
m_{1} \beta_{1}+m_{h} \beta_{h} \geq 1
$$

It is easy to verify that Eq. 83) holds if and only if $h=2$.

Case (c): $m_{1} \leq m_{h} \leq k_{2} m_{1}$. Similar to Case (b), $\psi \otimes \chi \prec$ $\varphi \otimes \omega$ holds for any small enough positive $\epsilon$ if and only if $h=2$.

Case (d): $m_{h}>k_{2} m_{1}$. Similar to Case (a), this also causes a contradiction.

Summarizing the above four cases, we obtain that $|\varphi\rangle$ should satisfy Eq. (70), which is equivalent to Eq. 69).

Now we turn to prove that the condition in Eq. (69) is also sufficient for partial entanglement recovery. Suppose that $|\varphi\rangle$ and $|\omega\rangle$ are with compact forms

$$
\varphi^{\downarrow}=\left(\beta_{1}^{\oplus m_{1}}, \beta_{2}^{\oplus m_{2}}\right)
$$

and

$$
\omega^{\downarrow}=\left(\gamma_{1}^{\oplus k_{1}-1}, \gamma_{1}-\epsilon, \gamma_{2}+\epsilon, \gamma_{2}^{\oplus k_{2}-1}\right),
$$

where

$$
\frac{\beta_{2}}{\beta_{1}}=\frac{\gamma_{2}}{\gamma_{1}} \text { and } \frac{1}{k_{2}} \leq \frac{m_{1}}{m_{2}} \leq k_{1} .
$$

Take $|\psi\rangle \in S^{o}(|\varphi\rangle)$. We shall prove that for a sufficiently small positive number $\epsilon$, the transformation of $|\psi\rangle \otimes|\chi\rangle$ to $|\varphi\rangle \otimes|\omega\rangle$ can be realized with certainty under LOCC.

By the assumptions $L_{u}(|\chi\rangle)=g_{u}(|\varphi\rangle)$ and $\psi \prec \varphi$, it is easy to verify that

$$
(\psi \otimes \chi)^{\downarrow}=\left(\psi^{\prime}, \psi^{\prime \prime}\right),
$$

where $\psi^{\prime}=\left(\psi \otimes \gamma_{1}^{\oplus k_{1}}\right)^{\downarrow}$ and $\psi^{\prime \prime}=\left(\psi \otimes \gamma_{2}^{\oplus k_{2}}\right)^{\downarrow}$. Similarly,

$$
(\varphi \otimes \chi)^{\downarrow}=\left(\varphi^{\prime}, \varphi^{\prime \prime}\right),
$$

where $\varphi^{\prime}=\left(\varphi \otimes \gamma_{1}^{\oplus k_{1}}\right)^{\downarrow}$ and $\varphi^{\prime \prime}=\left(\varphi \otimes \gamma_{2}^{\oplus k_{2}}\right)^{\downarrow}$.

By Eq. (84), it holds that $\gamma_{1} \beta_{2}=\gamma_{2} \beta_{1}$. Hence we also have

$$
\varphi^{\prime}=\left(\gamma_{1} \beta_{1}^{\oplus k_{1} m_{1}}, \gamma_{2} \beta_{1}^{\oplus k_{1} m_{2}}\right)
$$

and

$$
\varphi^{\prime \prime}=\left(\gamma_{1} \beta_{2}^{\oplus k_{2} m_{1}}, \gamma_{2} \beta_{2}^{\oplus k_{2} m_{2}}\right) .
$$


Similarly,

$$
(\varphi \otimes \omega)^{\downarrow}=\left(\varphi^{\prime}(\epsilon), \varphi^{\prime \prime}(\epsilon)\right),
$$

where

$$
\begin{aligned}
\varphi^{\prime}(\epsilon)= & {\left[\gamma_{1} \beta_{1}^{\oplus\left(k_{1}-1\right) m_{1}},\left(\gamma_{1}-\epsilon\right) \beta_{1}^{\oplus m_{1}},\right.} \\
& \left.\left(\gamma_{2}+\epsilon\right) \beta_{1}^{\oplus m_{1}}, \gamma_{2} \beta_{1}^{\oplus k_{1} m_{2}-m_{1}}\right]
\end{aligned}
$$

and

$$
\begin{aligned}
\varphi^{\prime \prime}(\epsilon)= & {\left[\gamma_{1} \beta_{2}^{\oplus m_{1} k_{2}-m_{2}},\left(\gamma_{1}-\epsilon\right) \beta_{2}^{\oplus m_{2}},\right.} \\
& \left.\left(\gamma_{2}+\epsilon\right) \beta_{2}^{\oplus m_{2}}, \gamma_{2} \beta_{2}^{\oplus\left(k_{2}-1\right) m_{2}}\right] .
\end{aligned}
$$

Note that Eqs. (90) and (91) are well-defined since we have Eq. (84). We have also assumed that $\epsilon$ in Eqs. (90) and (91) satisfies the following constraints:

$$
\left(\gamma_{1}-\epsilon\right) \beta_{1}>\left(\gamma_{2}+\epsilon\right) \beta_{1} \text { and }\left(\gamma_{2}+\epsilon\right) \beta_{2}<\left(\gamma_{1}-\epsilon\right) \beta_{2} .
$$

Since $\psi \triangleleft \varphi$, by Eqs. (85) and (86), applying Lemma 2.3 gives

$$
\psi^{\prime} \triangleleft \varphi^{\prime} \text { and } \psi^{\prime \prime} \triangleleft \varphi^{\prime \prime} .
$$

A careful observation caries out that $\varphi^{\prime}(\epsilon)$ and $\varphi^{\prime \prime}(\epsilon)$ are obtained by adding perturbations on $\varphi^{\prime}$ and $\varphi^{\prime \prime}$, respectively. So we have

$$
\psi^{\prime} \triangleleft \varphi^{\prime}(\epsilon) \text { and } \psi^{\prime \prime} \triangleleft \varphi^{\prime \prime}(\epsilon)
$$

for a sufficiently small positive number $\epsilon$.

Thus by Eqs. (85), (89), and (94), applying Lemma 7.2 gives

$$
\psi \otimes \chi \prec \varphi \otimes \omega .
$$

It is easy to see that $\omega \prec \chi$ and $\chi^{\downarrow} \neq \omega \downarrow$ providing the positive number $\epsilon$ small enough. In other words, $|\chi\rangle$ can do partial entanglement recovery for any transformation of $|\psi\rangle$ to $|\varphi\rangle$ such that $|\psi\rangle$ is in $S^{o}(|\varphi\rangle)$.

With that we complete the proof of Lemma 7.5

\section{Appendix C: Proof of Theorem 4.1}

For simplicity, we denote $I_{\psi, \varphi}$ and $D_{\psi, \varphi}$ by $I$ and $D$, respectively. We only need to show that for any $1 \leq l<$ $\operatorname{dim}(\psi) \operatorname{dim}(\chi)$, one of the following two cases holds:

Case 1: $e_{l}(\psi \otimes \chi)<e_{l}(\varphi \otimes \chi)$; or

Case 2: $e_{l}(\psi \otimes \chi)=e_{l}(\varphi \otimes \chi)$, but both sides are not related to $\chi^{i}(i \in D)$ and both of them remain unchanged by an arbitrary but small enough perturbations on $\chi^{i}(i \in D)$. Here we should point out that $\sum \chi^{i}$ is supposed to be kept as a constant for each $i \in D$ during the perturbations to guarantee that $|\chi\rangle$ is a valid quantum state.

For this purpose, we rewrite $e_{l}(\psi \otimes \chi)$ as follows:

$$
e_{l}(\psi \otimes \chi)=\sum_{i=1}^{m} \sum_{j=1}^{m} e_{l_{i, j}}\left(\psi^{i} \otimes \chi^{j}\right),
$$

where $\sum_{i, j} l_{i, j}=l$ and $0 \leq l_{i, j} \leq \operatorname{dim}\left(\psi^{i} \otimes \chi^{j}\right)$.

It is easy to see that

$$
\begin{aligned}
e_{l}(\psi \otimes \chi) & =\sum_{i, j} e_{l_{i, j}}\left(\psi^{i} \otimes \chi^{j}\right) \\
& \leq \sum_{i, j} e_{l_{i, j}}\left(\varphi^{i} \otimes \chi^{j}\right) \\
& \leq e_{l}(\varphi \otimes \chi),
\end{aligned}
$$

where the first inequality follows from $\psi^{i} \otimes \chi^{j} \prec \varphi^{i} \otimes \chi^{j}$ and the second one follows from the definition of $e_{l}(\varphi \otimes \chi)$.
If one of these inequalities is strict, then Case 1 holds, and the proof is completed; otherwise we only need to prove that Case 2 holds.

More precisely, we only need to show that if $e_{l}(\psi \otimes \chi)=$ $e_{l}(\varphi \otimes \chi)$ then for any $1 \leq i \leq m$ and $j \in D, l_{i, j}$ can only take two values: 0 or $\operatorname{dim}\left(\psi^{i} \otimes \chi^{j}\right)$. Notice that $I \cup D=\{1, \ldots, m\}$ and $I \cap D=\emptyset$. It suffices to prove $l_{i, j} \in\left\{0, \operatorname{dim}\left(\psi^{i} \otimes \chi^{j}\right)\right\}$ for two cases: (1) $i \in I, j \in D$, and (2) $i \in D, j \in D$.

Let us consider the case when $i \in I$ and $j \in D$ first. By Eq. (10), we have

$$
\varphi^{i} \otimes \chi^{j} \sqsubset \varphi^{j} \otimes \chi^{i}, \text { for all } i \in I \text { and } j \in D .
$$

That is, the values of the extreme components of $\varphi^{i} \otimes \chi^{j}$ are strictly bounded by those of $\varphi^{j} \otimes \chi^{i}$. Thus, we have

$$
\left(\varphi^{i} \otimes \chi^{j}\right)_{1}^{\downarrow}<\left(\varphi^{j} \otimes \chi^{i}\right)_{1}^{\downarrow}
$$

and

$$
\left(\varphi^{i} \otimes \chi^{j}\right)_{1}^{\uparrow}>\left(\varphi^{j} \otimes \chi^{i}\right)_{1}^{\uparrow} .
$$

Hence by the assumption that all inequalities in Eq. (96) hold with equalities and the definition of $e_{l}(\varphi \otimes \chi)$, together with the above two equations, we have

$$
l_{j, i}=0 \Rightarrow l_{i, j}=0
$$

and

$$
l_{j, i}=\operatorname{dim}\left(\psi^{j} \otimes \chi^{i}\right) \Rightarrow l_{i, j}=\operatorname{dim}\left(\psi^{i} \otimes \chi^{j}\right) .
$$

So, in order to prove $l_{i, j} \in\left\{0, \operatorname{dim}\left(\psi^{i} \otimes \chi^{j}\right)\right\}$ in the case of $i \in$ $I$ and $j \in D$, we only need to show that $l_{j, i} \in\left\{0, \operatorname{dim}\left(\psi^{j} \otimes\right.\right.$ $\left.\left.\chi^{i}\right)\right\}$ for $i \in I$ and $j \in D$. Or equivalently, to show $l_{i, j} \in$ $\left\{0, \operatorname{dim}\left(\psi^{i} \otimes \chi^{j}\right)\right\}$ for $i \in D$ and $j \in I$. (Here we interchange the indices $i$ and $j$ for convenience.)

So combining this with the case of $i \in D$ and $j \in D$, the only thing left to be proven is that

$l_{i, j} \in\left\{0, \operatorname{dim}\left(\psi^{i} \otimes \chi^{j}\right)\right\}$ for all $i \in D$ and $1 \leq j \leq m$.

By Eq. (11) and Lemma 2.3 we have

$$
\psi^{i} \otimes \chi^{j} \triangleleft \varphi^{i} \otimes \chi^{j} \text { for all } i \in D \text { and } 1 \leq j \leq m .
$$

If there exist $s \in D$ and $1 \leq t \leq m$ such that $0<l_{s, t}<$ $\operatorname{dim}\left(\psi^{s} \otimes \chi^{t}\right)$, then by Eq. (99) we have

$$
e_{l_{s, t}}\left(\psi^{s} \otimes \chi^{t}\right)<e_{l_{s, t}}\left(\varphi^{s} \otimes \chi^{t}\right) .
$$

It follows that the first inequality in Eq. (96) strictly holds, which contradicts $e_{l}(\psi \otimes \chi)=e_{l}(\varphi \otimes \chi)$. So Eq. (98) holds. With that we complete the proof of Theorem 4.1.

\section{ACKNOWLEDGEMENT}

The authors are very grateful to the anonymous referees for their detailed comments and instructive suggestions that helped to improve the presentation of this paper greatly. In particular, they pointed out a technical error in the proof of Lemma 2.2 in an earlier version of this paper. The simple proof of statement (2) of Lemma 2.2 in this version is due to one of them. The algorithmic approach to partial entanglement recovery has been motivated heavily by their suggestions. The authors are also indebted to the colleagues in the Quantum Computation and Quantum Information Research Group for many useful discussions. A special acknowledgement is given to Yongzhi Cao for his careful reading of this paper. 


\section{REFERENCES}

[1] C. H. Bennett and G. Brassard, Proceedings of IEEE International Conference on Computers, Systems, and Signal Processing, pp. 175179, IEEE, New York, 1984.

[2] C. H. Bennett and S. J. Wiesner, "Communication via one- and twoparticle operators on Einstein-Podolsky-Rosen states," Phys. Rev. Lett., vol. 69, no. 20, pp. 2881-2884, 1992.

[3] C. H. Bennett, G. Brassard, C. Crepeau, R. Jozsa, A. Peres, and W. K. Wootters, "Teleporting an unknown quantum state via dual classical and Einstein-Podolsky-Rosen channels," Phys. Rev. Lett., vol. 70, no. 13, pp. 1895-1899, 1993.

[4] M. A. Nielsen and I. L. Chuang, Quantum Computation and Quantum Information, Cambridge University Press, Cambridge, 2000.

[5] C. H. Bennett, H. J. Bernstein, S. Popescu, and B. Schumacher, "Concentrating partial entanglement by local operations," Phys. Rev. A, vol. 53, no. 4, pp. 2046-2052, 1996.

[6] M. A. Nielsen, "Conditions for a class of entanglement transformations," Phys. Rev. Lett., vol. 83, no.2, pp. 436-439, 1999.

[7] A. W. Marshall and I. Olkin, Inequalities: Theory of Majorization and Its Applications, New York, American: Academic Press, 1979.

[8] P. M. Alberti and A. Uhlmann, Stochasticity and Partial Order: Doubly Stochastic Maps and Unitary Mixing, Dordrecht, Boston, 1982.

[9] C. H. Bennett, P. W. Shor, J. A. Smolin, and A. V. Thapliyal, "Entanglement-assisted classical capacity of a quantum channel and the reverse Shannon theorem," IEEE Trans. Inf. Theory, vol. 48, no. 10, pp. 2637-2655, Oct. 2002.

[10] F. Morikoshi, "Recovery of entanglement lost in entanglement manipulation," Phys. Rev. Lett., vol. 84, no. 14, pp. 3189-3192, 2000.

[11] F. Morikoshi and M. Koashi, "Deterministic entanglement concentration," Phys. Rev. A, vol. 64, no. 2, art. 022316, 2001.

[12] S. Bandyopadhyay, V. Roychowdhury, and F. Vatan, "Partial recovery of entanglement in bipartite-entanglement transformations," Phys. Rev. A, vol. 65, no. 4, art. 040303 (Rapid Communications), 2001.

[13] S. Bandyopadhyay, V. Roychowdhury, and F. Vatan, "Partial recovery of entanglement in bipartite-entanglement transformations". Available online: http://arxiv.org/abs/quant-ph/0105019

[14] S. Bandyopadhyay, and V. Roychowdhury, "Efficient entanglementassisted transformation for bipartite pure states," Phys. Rev. A, vol. 65, no. 4, art. 042306, 2002.

[15] S. Bandyopadhyay, V. Roychowdhury, and U. Sen, "Classification of nonasymptotic bipartite pure-state entanglement transformations," Phys. Rev. A, vol. 65, no. 5, art. $052315,2002$.

[16] H.-K. Lo and S. Popescu, "Concentrating entanglement by local actions: beyond mean values," Phys. Rev. A, vol. 63, no. 2, art. 022301, 2001. Available online: http://arxiv.org/abs/quant-ph/9707038

[17] G. Vidal, "Entanglement of pure states for a single copy," Phys. Rev. Lett., vol. 83, no. 5, pp. 1046-1049, 1999.

[18] D. Jonathan and M. B. Plenio, "Entanglement-assisted local manipulation of pure quantum states," Phys. Rev. Lett., vol. 83, no. 17, pp. 3566-3569, 1999.

[19] R. Y. Duan, Y. Feng, X. Li, and M. S. Ying, "Trade-off between multiple-copy transformation and entanglement cataysis," Phys. Rev. A, vol. 71, no. 6, art. 062306, 2005. Available online: http://www.arXiv.org/abs/quant-ph/0312010

[20] R. Y. Duan, Y. Feng, X. Li, and M. S. Ying, "Multiplecopy entanglement transformation and entanglement cataysis," Phys. Rev. A, vol. 71, no. 4, art. 042319, 2005. Available online: http://www.arXiv.org/abs/quant-ph/0404148

[21] R. Y. Duan, Y. Feng, and M. S. Ying, "An equivalence of entanglementassisted transformation and multiple-copy transformation." Available online: http://www.arXiv.org/abs/quant-ph/0404046

[22] X. M. Sun, R. Y. Duan, and M. S. Ying, "The existence of quantum entanglement catalysts," IEEE Trans. Inf. Theory, vol. 51, no. 1, pp. 75-80, Jan. 2005.

[23] X. L. Feng, Z. Y. Wang, and Z. Z. Xu, "Mutual catalysis of entanglement transformations for pure entangled states," Phys. Rev. A, vol. 65, no. 2, art. 022307, 2002

[24] H. Edelsbrunner, J. O'Rourke, and R. Seidel, "Constructing arrangements of lines and hyperplanes with applications," SIAM J. Comput., vol. 15 , pp. 341-363, 1986.

[25] N. Karmarkar, "A new polynomial-time algorithm for linear programming," Combinatorica, vol. 4, pp. 373-395, 1984.

[26] N. Megiddo, "Linear programming in linear time when the dimension is fixed," J. ACM, vol. 31, pp. 114-127, 1984. 